IGC-11/7-1

\title{
Exact half-BPS string-junction solutions in six-dimensional supergravity ${ }^{1}$
}

\author{
Marco Chiodaroli $^{a}$, Eric D’Hoker ${ }^{b}$, Yu Guo ${ }^{b}$, Michael Gutperle ${ }^{b}$ \\ a Institute for Gravitation and the Cosmos, \\ The Pennsylvania State University, University Park, PA 16802, USA \\ mchiodar@gravity.psu.edu \\ $b$ Department of Physics and Astronomy \\ University of California, Los Angeles, CA 90095, USA \\ dhoker, guoyu, gutperle@physics.ucla.edu;
}

\begin{abstract}
We construct $S O(2,1) \times S O(3)$-invariant half-BPS solutions in six-dimensional $(0,4)$ supergravity with $m$ tensor multiplets. The space-time manifold of each one of these solutions consists of an $A d S_{2} \times S^{2}$ warped over a Riemann surface $\Sigma$ with boundary. The most general local solution is parametrized by one real harmonic function, and $m+2$ holomorphic functions which are subject to a quadratic constraint and a hermitian inequality, both of which are manifestly $S O(2, m)$ invariant. Imposing suitable conditions on these harmonic and holomorphic functions, we construct globally regular supergravity solutions with $N$ distinct $A d S_{3} \times S^{3}$ asymptotic regions and contractible $\Sigma$. These solutions have an intricate moduli space, whose dimension equals $2(m+1) N-m-2$ and matches the counting of three-form charge vectors and unattracted scalars of the tensor multiplet. Exact explicit formulas for all supergravity fields are obtained in terms of the moduli.

Our solutions give the near-horizon geometries for junctions of $N$ self-dual strings in six dimensions, and are holographic duals to CFTs defined on $N$ half-planes which share a common interface line. For $m=5$, the solutions lift to quarter-BPS solutions for six-dimensional $(4,4)$ supergravity.
\end{abstract}

\footnotetext{
${ }^{1}$ This work is supported in part by NSF grants PHY-08-55356 and PHY-07-57702.
} 


\section{Contents}

1 Introduction $\quad 4$

1.1 Summary of results and organization . . . . . . . . . . . . . . . . 6

2 Review of six-dimensional supergravities $\quad 10$

2.1 The $(0,4)$ theory . . . . . . . . . . . . . . . . . . . . 11

2.1.1 Chiral truncation of the $(4,4)$ theory . . . . . . . . . . . 11

2.1.2 Possible truncations of the $(0,8)$ and $(2,6)$ theories ? . . . . . . . 12

2.2 Scalar fields . . . . . . . . . . . . . . . . . . . . . . 12

2.3 Anti-symmetric tensor fields . . . . . . . . . . . . . . . . . . . . . . . . . . . . . . . 13

2.4 Bianchi identities . . . . . . . . . . . . . . . . . . . . . 14

2.5 Field equations for bosonic fields . . . . . . . . . . . . . . . . 14

2.6 Supersymmetry transformation for fermionic fields . . . . . . . . . . . 15

3 Ansatz and reduced BPS equations 16

3.1 The $S O(2,1) \times S O(3)$-invariant Ansatz . . . . . . . . . . . . . 16

3.2 Killing spinor basis . . . . . . . . . . . . . . . . . . . . . . . . . . . . . . . . . . .

3.3 Derivation of the reduced BPS equations . . . . . . . . . . . . . . 18

3.4 Chiral form of the reduced BPS equations . . . . . . . . . . . . . . . . 19

4 Solving the reduced BPS equations $\quad 21$

4.1 Algebraic relation between $\xi$ and $\psi \ldots \ldots$. . . . . . . . . . . . 21

4.2 Identifying the harmonic function $H \ldots \ldots$. . . . . . . . . . . . . . . . 22

4.3 Algebraic projector conditions . . . . . . . . . . . . . . . . . . . 23

4.4 Solving the projector conditions . . . . . . . . . . . . . . 23

4.5 Solving the remaining differential equations . . . . . . . . . . . . . . 24

4.6 Solving for $p_{a}^{i r}$ and $\tilde{g}_{a}^{r} \ldots \ldots \ldots \ldots \ldots \ldots$

5 Constructing the local solution $\quad 29$

5.1 Summary of the solutions to the BPS equations . . . . . . . . . . . . . 29

5.2 Solving for $V$ and $|X| \ldots \ldots \ldots \ldots \ldots$

5.2 .1 Solving for $V_{I}^{i} \ldots \ldots \ldots \ldots$

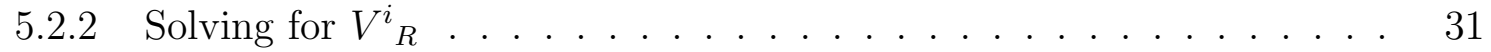

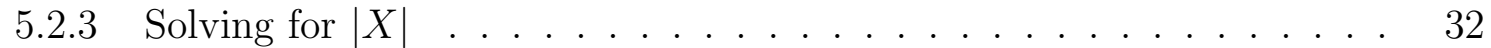

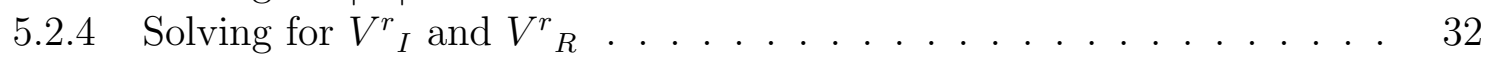

5.3 Calculating the flux potentials . . . . . . . . . . . . . . 33

5.4 Summary of the local solution . . . . . . . . . . . . . . . . . . . 34

6 Topology and regularity assumptions 36

6.1 Basic regularity assumptions . . . . . . . . . . . . . . . 36

6.2 Implications on the (locally) holomorphic data . . . . . . . . . . . . . 37 
$\begin{array}{lll}7 & \text { Regular locally asymptotically } A d S_{3} \times S^{3} \text { solutions } & \mathbf{3 8}\end{array}$

7.1 Solving the regularity conditions on $H \ldots \ldots \ldots \ldots$

7.2 Solving the regularity conditions on $\Lambda^{A} \ldots \ldots \ldots \ldots$

7.3 Regularity at the poles of $\lambda^{A} \ldots \ldots \ldots \ldots$

7.4 Solving the constraints . . . . . . . . . . . . . . . . . . 39

7.5 Asymptotic behavior of the metric in $A d S_{3} \times S^{3}$ regions . . . . . . . . . 40

7.6 Calculation of the charges and conservation . . . . . . . . . . . 41

7.7 Attractor behavior of scalars fields in $A d S_{3} \times S^{3}$ regions $\ldots \ldots \ldots 4$

$7.8 \mathrm{SL}(2, \mathrm{R})$ transformations on equations and solutions . . . . . . . . . . . 42

7.9 Counting moduli of the solutions . . . . . . . . . . . . . . 43

7.10 Counting physical parameters of the solutions . . . . . . . . . 43

8 Numerical solutions, $N=2,3,4,5 \quad \mathbf{4 4}$

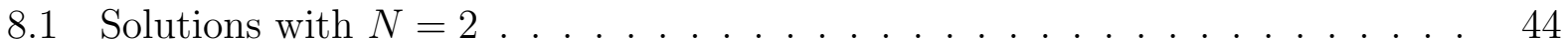

8.2 Solutions with $N=3 \ldots \ldots \ldots \ldots \ldots$. . . . . . . . . . . . . . 44

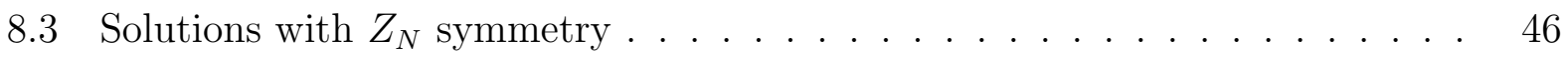

8.4 Numerical solutions with $Z_{4}$ and $Z_{5}$ symmetry $\ldots \ldots \ldots . \ldots 48$

9 Maximal families of regular solutions for all $m, N \quad \mathbf{5 0}$

9.1 Light-cone like solution of the first constraint . . . . . . . . . . . . . 50

9.2 Solving the second constraint . . . . . . . . . . . . . 51

9.3 Parametrization of solutions by "auxiliary poles" . . . . . . . . . . . . 51

9.4 Relating covariant data to auxiliary pole data . . . . . . . . . . . . 52

9.5 Counting data and explicit parametrization of solutions . . . . . . . . 53

9.6 Relation to the solutions of $[31] \ldots \ldots \ldots \ldots \ldots$

$\begin{array}{ll}10 \text { Discussion } & 56\end{array}$

$\begin{array}{ll}\text { A Notations and Conventions } & \mathbf{5 8}\end{array}$

A.1 Six-dimensional Minkowski space-time . . . . . . . . . . . . . . . 58

A.2 $S O(5, m) /(S O(5) \times S O(m))$ coset space notations . . . . . . . . . 59

A.3 $S O(1,5)$-gamma matrices $\ldots \ldots \ldots \ldots \ldots \ldots$

A.4 $S O(5)$-gamma matrices . . . . . . . . . . . . . . . . 60

A.5 Choice of a spinor basis in $A d S_{2} \times S^{2} \times \Sigma \ldots \ldots \ldots$. . . . . . . 61

$\begin{array}{ll}\text { B Reducing the BPS equations } & 62\end{array}$

B.1 Reduction of the gravitino BPS equation . . . . . . . . . . . . . . 62

B.2 Reduction of the $\chi^{r \alpha}$ BPS equation . . . . . . . . . . . . . 64 


\section{Introduction}

Over the past few years, considerable progress has been made on the construction of exact half-BPS classical solutions to Type IIB and M-theory supergravity. Research motivated by studies in gauge/gravity duality [1, 2, 3] (for reviews, see e.g. [4, 5]) has focused on solutions whose space-time manifold contains a (warped) factor of an $A d S$ space, and on so-called bubbling solutions. By construction, all these solutions have non-trivial isometries, and an asymptotic symmetry which coincides with the asymptotic symmetry of the maximally supersymmetric solution.

A general classification of such half-BPS solutions for both Type IIB and M-theory supergravity in terms of conformal superalgebras and their subalgebras was proposed in [6]. Many of the resulting cases admit immediate interpretations in terms of intersecting branes, and are holographic duals to gauge theory states with defects of various dimensions. For example, Type IIB solutions which are holographic duals to states of $\mathcal{N}=4$ super-Yang-Mills (SYM) theory in $3+1$-dimensional space-time include the following: (1) the bubbling solutions of [7], which are invariant under the superalgebra $P S U(2 \mid 2)^{2}$ and dual to local half-BPS operators; (2) the solutions of [8], which are invariant under the superalgebra $O S p\left(4^{*} \mid 4\right)$ and dual to straight Wilson lines (see also [9, 10, 11]); (3) the half-BPS Janus solutions of $[12,13]$, which are invariant under $O S p(4 \mid 4, \mathbf{R})$, are dual to a planar $2+1$-dimensional interface or defect (see also $[14,15,16,17,18]$ ), and provide supersymmetric generalizations to the original Janus solution of [19]. In M-theory, bubbling solutions were derived in [7], while a wealth of half-BPS solutions with $A d S$ factor is available in $[20,21,22,23,24]$.

Supersymmetry provides a powerful tool for the construction of explicit solutions, as second order field equations may be replaced by first order BPS equations. In the case of defect solutions with 16 supersymmetries, the BPS equations in Type IIB and M-theory suffice to solve the full Bianchi and field equations. The space-time structure of the resulting half-BPS solutions to Type IIB and M-theory is of the form,

$$
A d S_{p_{0}} \times S^{p_{1}} \times S^{p_{2}} \times \Sigma
$$

where $\Sigma$ is a Riemann surface with boundary over which the product $A d S_{p_{0}} \times S^{p_{1}} \times S^{p_{2}}$ is warped, while the total dimension $p_{0}+p_{1}+p_{2}+2$ equals 10 for Type IIB and 11 for M-theory. All these solutions have co-homogeneity 2, following the definition of [25]. Remarkably, although various integrable systems on $\Sigma$ arise during the course of the analysis of the BPS equations [26], the final solutions are invariably expressible in terms of holomorphic functions on $\Sigma$, parametrized by strata of finite-dimensional moduli spaces.

Supersymmetry can still be a powerful tool with fewer supersymmetries. One class of 
examples $^{1}$ are Janus-like solutions with 8 supersymmetries arise by considering Type IIB supergravity on the following space-times involving a $K 3$ surface,

$$
A d S_{2} \times S^{2} \times K 3 \times \Sigma
$$

where the product $A d S_{2} \times S^{2} \times K 3$ is warped over the Riemann surface $\Sigma$ with boundary. The solutions of [31, 32] (see also [33, 34, 35, 36, 37]) are locally asymptotic to the $A d S_{3} \times S^{3} \times K 3$ vacuum solution, which has 16 supersymmetries. Although less supersymmetric than their counterparts of (1.1), these solutions are still expressible in terms of holomorphic functions on $\Sigma$ and parametrized by interesting moduli spaces.

However, the solutions of $[31,32]$ did not turn on the moduli and fluxes of the $K 3$ factor. In principle this can be done using a ten-dimensional Ansatz, but it will be simpler to consider this problem directly within the framework of the six-dimensional supergravity obtained by the compactification of Type IIB on $K 3$. The moduli of the $K 3$ manifold arise as scalar fields which, together with the dilaton and axion, live in a coset manifold.

Six-dimensional supergravity admits dyonic string solutions preserving four supersymmetries which are analogues to $(p, q)$ strings in ten dimensions. In [38, 39] it was shown that in ten dimensions there exist BPS configurations where three $(p, q)$ strings join at a point, and that such configurations can be combined to construct networks of strings.

A similar analysis was carried out in six dimensions in [37], finding that there exist supersymmetric junctions with an arbitrary number of dyonic strings, provided that the angles between the strings are adjusted according to their charges. Naturally, the solutions of $[31,32]$ were interpreted as the smooth near-horizon geometries produced by the backreaction of these junctions of dyonic strings.

In this paper, we shall construct exactly the general solution with 8 supersymmetries to the six-dimensional chiral $(0,4)$ - or "Type 4b" - supergravity theory which has 16 supersymmetries. The $(0,4)$ theory is in many respects the six-dimensional analogue of tendimensional Type IIB supergravity. The $(0,4)$ theory was constructed by Romans [40] (see also $[41,42])$, and consists of a supergravity multiplet together with $m$ tensor supermultiplets. Classically, all values of $m$ are allowed, but anomaly cancellation restricts $m$ to the values 5 or 21 . The scalars live in the $S O(5, m) /(S O(5) \times S O(m))$ coset manifold. For $m=5$, the $(0,4)$ theory may be obtained as a chiral truncation of the $(4,4)$ six-dimensional supergravity theory with 32 supersymmetries, which was constructed in [43]. The $m=21$ supergravity is given by Kaluza-Klein compactification of Type IIB on $K 3$, but it appears unknown, at present, whether this truncation is consistent or not. ${ }^{2}$

\footnotetext{
${ }^{1}$ Other examples are bubbling $A d S_{3}$ solutions, see e.g. [27, 28, 29, 30].

${ }^{2}$ We thank the referee for stressing this point.
} 
The $(0,4)$ supergravity admits a vacuum solution with space-time manifold $A d S_{3} \times S^{3}$ and the maximal number of 16 supersymmetries. Its isometries are the $S O(2,2) \times S O(4)$ maximal bosonic subalgebra of the full $P S U(1,1 \mid 2)^{2}$ symmetry superalgebra. The vacuum solution is analogous to the $A d S_{5} \times S^{5}$ solution to Type IIB with 32 supersymmetries. The space-time manifold of six-dimensional half-BPS Janus solutions is of the form,

$$
A d S_{2} \times S^{2} \times \Sigma
$$

where the product $A d S_{2} \times S^{2}$ is warped over the Riemann surface $\Sigma$. The solutions are invariant under the isometry $S O(2,1) \times S O(3)$ which is the maximal bosonic subalgebra of the full $P S U(1,1 \mid 2)$ symmetry superalgebra of the six-dimensional Janus solutions. Contrarily to the half-BPS Janus slicing of $A d S_{5} \times S^{5}$ in Type IIB, the warped space-time of (1.3) provides an essentially unique $A d S$-slicing of the vacuum solution $A d S_{3} \times S^{3}$. Asymptotically $A d S_{3} \times S^{3}$ bubbling solutions with geometry $S^{1} \times S^{1} \times \mathbf{R} \times \Sigma_{3}$ where $\Sigma_{3}$ is a three-dimensional base, were obtained in $[27,36,28]$. Together with these bubbling solutions, the $A d S_{3} \times S^{3}$ Janus solutions account for the most general presently known class of half-BPS solutions with $A d S_{3} \times S^{3}$ asymptotics in six-dimensional supergravity.

\subsection{Summary of results and organization}

Since this paper is relatively long and technical, we shall present here a brief summary of our results. There are two parts to the solution; one local, the other global.

First, we shall derive a complete local solution, characterized by one real harmonic function and $m+2$ holomorphic differential one-forms on the Riemann surface $\Sigma$. Such a local solution solves the BPS conditions, the Bianchi identities, and the field equations point-wise on $\Sigma$. The local solution will be obtained in several steps. In Section 3 the BPS equations will be reduced using the $S O(2,1) \times S O(3)$-invariant Ansatz for the metric,

$$
d s^{2}=f_{1}^{2} d s_{A d S_{2}}^{2}+f_{2}^{2} d s_{S^{2}}^{2}+d s_{\Sigma}^{2}
$$

as well as corresponding Ansätze for the scalar and anti-symmetric tensor fields. Solving the BPS equations in Section 4, we shall find that the scalar fields are restricted to live on a $S O(2, m) /(S O(2) \times S O(m))$ sub-manifold of the full $S O(5, m) /(S O(5) \times S O(m))$ scalar coset, and that the anti-symmetric tensor fields are restricted accordingly. However, the solutions to the BPS equations do not automatically solve the Bianchi and field equations. In Section 5, the local solutions to the BPS equations are combined with the Bianchi identities, and used to produce solutions to the full set of Bianchi and field equations. These solutions are parametrized by a single real harmonic function $H$, and $m+2$ holomorphic one-forms 
$\Lambda^{A}$ with $A=1, \cdots, m+5$, and $\Lambda^{3}=\Lambda^{4}=\Lambda^{5}=0$, subject to the relations, ${ }^{3}$

$$
\begin{aligned}
& \Lambda \cdot \Lambda=2(\partial H)^{2} \\
& \Lambda \cdot \bar{\Lambda} \geq 2|\partial H|^{2}
\end{aligned}
$$

where $\eta$ is the $S O(5, m)$-invariant metric. The expressions for the metric factors $f_{1}, f_{2}$, and $d s_{\Sigma}^{2}$, as well as for the scalars, anti-symmetric tensor fields and associated charges of the solution may be found in Section 5.4.

Second, to obtain globally well-defined and regular solutions, which are locally asymptotically $A d S_{3} \times S^{3}$, the harmonic function $H$ and the holomorphic one-forms $\Lambda^{A}$ must be subject to certain topology and regularity conditions, which will be spelled out in Section 6 . A first topological condition requires the boundary of the Riemann surface $\Sigma$ to correspond to a vanishing volume for the $S^{2}$ fiber, so that $f_{2}=0$ on $\partial \Sigma$, and $f_{2} \neq 0$ in the interior of $\Sigma$. For simplicity in the present paper, we shall assume that $\partial \Sigma$ consists of a single connected component, though we know from [32] that this assumption may be easily relaxed, and that $\partial \Sigma$ can support multiple connected boundary components. A second topological condition requires that the space-time manifold will have $N$ of asymptotic regions where the geometry is locally asymptotically $A d S_{3} \times S^{3}$. Finally, the solution should otherwise be smooth.

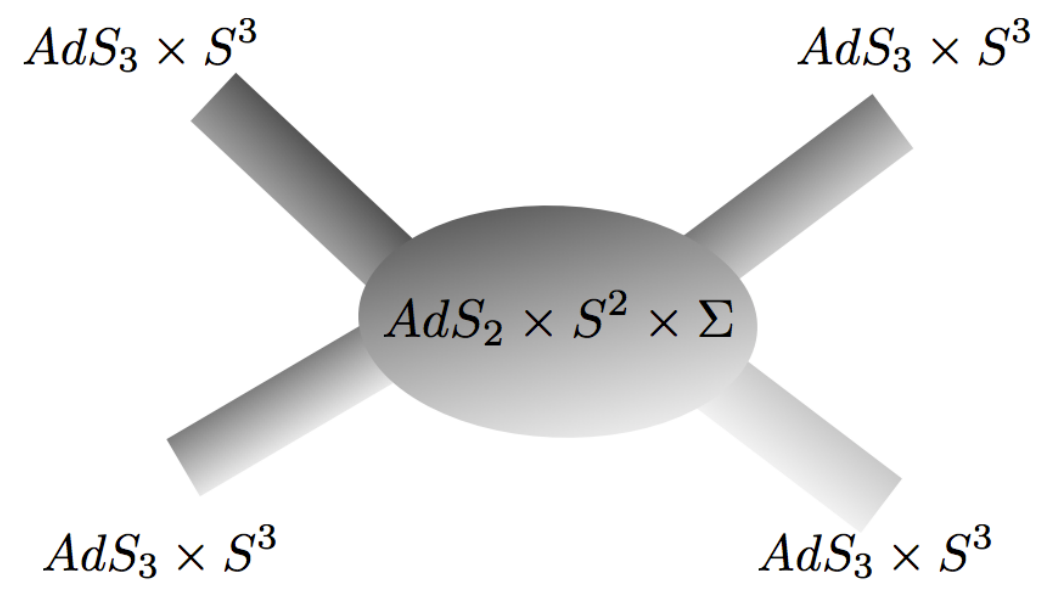

Figure 1: Global structure of the $N=4$ solutions.

Parameterizing $\Sigma$ by the upper half-plane, the above topological and regularity conditions are solved by a harmonic function $H$ and $m+2$ holomorphic one-forms $\Lambda^{A}$ given by,

$$
H(w, \bar{w})=\sum_{n=1}^{N}\left(\frac{i c_{n}}{w-x_{n}}-\frac{i c_{n}}{\bar{w}-x_{n}}\right)
$$

\footnotetext{
${ }^{3}$ Throughout, we shall use the $S O(5, m)$-invariant metric $\eta=\operatorname{diag}\left(I_{5},-I_{m}\right)$ to raise and lower indices $K^{A}=\eta^{A B} K_{B}$, for $\mathbf{C}^{5, m}$ vectors $K$, and to form their inner product defined by $K \cdot L \equiv \eta_{A B} K^{A} L^{B}$.
} 


$$
\Lambda^{A}(w)=\sum_{n=1}^{N}\left(\frac{-i \kappa_{n}^{A}}{\left(w-x_{n}\right)^{2}}+\frac{-i \mu_{n}^{A}}{w-x_{n}}\right) d w
$$

where the positions of the poles $x_{n}$ and their residues $c_{n}, \kappa_{n}^{A}, \mu_{n}^{A}$ are real, with $c_{n}>0$, and restricted by the fact that $H$ and $\Lambda^{A}$ must obey both relations of (1.5). To each pole $x_{n}$ corresponds a distinct asymptotic $A d S_{3} \times S^{3}$ region, where $\mu_{n}^{A}$ gives the $m+2$-dimensional vector of three-form charges ${ }^{4}$ on the corresponding asymptotic three-cycles $S^{3}$. There are also $2 m$ scalar expectation values associated with each asymptotic $A d S_{3} \times S^{3}$ region. The attractor mechanism fixes $m$ of these in terms of the charges $\mu_{n}^{A}$. The values of the other $m$ scalars are related to the residues $\kappa_{n}^{A} / c_{n}$, and are (locally) arbitrary. Thus, each asymptotic region has $2 m+2$ moduli. Charge conservation demands that the sum of the charge vectors $\mu_{n}^{A}$ vanishes. Consequently, the total number of independent physical parameters coming from all asymptotic regions is,

$$
\text { number of independent real moduli }=2(m+1) N-(m+2)
$$

It will be shown in Section 7 that the number of physically independent moduli of (1.7) precisely matches the number of free parameters in the solution (1.6) subject to (1.5).

For general values of $m$ and $N$, the constraints imposed by (1.5) on the parameters $x_{n}, c_{n}, \kappa_{n}^{A}$, and $\mu_{n}^{A}$ consist of nested quadratic relations, and are highly non-trivial to solve analytically. Therefore, in Section 8 , we shall begin by exhibiting solutions in a number of special cases, namely by solving (1.5) numerically for $N=3,4,5$. These results demonstrate that regular solutions with asymptotic $A d S_{3} \times S^{3}$ regions do exist, and may be obtained by straightforward numerical analysis.

Finally, in Section 9, we shall present a completely explicit analytical solution with the full number of free parameters given in (1.7), for general $m$, and general number $N$ of asymptotic $A d S_{3} \times S^{3}$ regions. To do so, a light-cone like parametrization of the functions $\lambda^{A}=\Lambda^{A} / \partial H$ is used to solve the first constraint of (1.6) in a rational manner. The corresponding holomorphic light-cone functions may be parametrized explicitly by $2 N-2$ auxiliary poles and associated residues, in terms of which the second constraint of (1.6) reduces to a set of Schwarz identities, which are found to be easily satisfied. For $m \leq 2$, our solutions are found to reduce precisely to the solutions obtained in [31, 32], as should have been expected.

The solutions constructed in this paper are to Type 4b six-dimensional supergravity with $m$ tensor multiplets. Certain classes of these solutions are known to uplift to exact solutions of the full ten-dimensional Type IIB supergravity. They include all solutions with $m=5$,

\footnotetext{
${ }^{4}$ This counting holds when $\Sigma$ is simply connected; otherwise additional homology three-cycles and charges will arise, which must be included in the counting towards the total number of charges.
} 
since they can be lifted to Type IIB solutions via the consistent truncation of this theory on the zero modes of $T^{4}$. They also include all solutions for $m=21$ for which the scalars take values in a $S O(2,2) / S O(2) \times S O(2)$ submanifold of the full $S O(5,21) / S O(5) \times S O(21)$ coset, where no moduli of the K3 are turned on and the 2-form fields restricted accordingly [31]. Whether the general solutions, beyond these two special cases, also lift to exact solutions of Type IIB is unknown to us since it is, at present, unknown whether six dimensional Type 4b theory with $m=21$ arises as a consistent truncation of Type IIB on $K 3$, or not. All of our solutions are, however, approximate solutions to Type IIB in the usual sense of KaluzaKlein reduction, provided the scale of the solution is much larger than the scale of the $K 3$ compactification manifold. Since each one of our solutions, for given asymptotic charges $\mu_{n}$ with $\mu_{n} \cdot \mu_{n}>0$, is completely regular as a 6 -dimensional space-time, there will always be a sufficiently small scale of the $K 3$ compactification (determined by the moduli of the solution) for which the solution is a reliable approximation to a full Type IIB solution with the same charge assignments.

The holographic interpretation of our solutions is given as the dual of interfaces between two CFTs, or more general as a junction of an arbitrary number of CFTs. The discussion parallels the one given in [31] which we will briefly review in the following. Each one of the $N$ asymptotic $A d S_{3} \times S^{3}$ regions is dual to a $1+1$-dimensional CFT defined on a half line $(x$ time). Each CFT is associated with the near-horizon limit of a half-BPS dyonic string in the six-dimensional supergravity carrying the same charges. The CFTs are joined at a zero-dimensional defect which on the gravity side can be thought of as the boundary of the $A d S_{2}$ factor. Hence the solution is the holographic dual of a junction of $N$ CFTs glued together at a one dimensional defect. The values of the un-attracted scalars in each asymptotic region correspond to exactly marginal deformations (i.e. moduli) of each CFT. This construction generalizes the Janus solution which has only two asymptotic AdS regions and corresponds to an interface of two CFTs where the moduli can jump when crossing the one dimensional interface.

Since each asymptotic $A d S_{3} \times S^{3}$ region is associated with half-BPS dyonic string the $1 / 2$ BPS solutions presented in this paper can be viewed as near horizon limit of $1 / 4$ BPS junctions of strings in 6 dimensional flat space. The supersymmetry gets enhanced in the near horizon limit. The existence of exact solutions of junctions of CFTs makes it possible to holographically calculate important observables such as the entanglement entropy and reflection and transmission coefficients. It might be possible that our solutions have some relevance for the description of junctions of quantum wires. 


\section{Review of six-dimensional supergravities}

In this section, we shall give a brief overview of supergravity theories in six space-time dimensions, with various degrees of supersymmetry.

In six dimensions, a Weyl spinor is complex but self-conjugate. The Majorana condition cannot be imposed consistently on a single Weyl spinor; however, the symplectic Majorana condition may be imposed consistently on pairs of Weyl spinors.

As a result, supergravity theories may be distinguished by a pair of integers $\left(n_{+}, n_{-}\right)$ where $n_{ \pm}$count the number of Weyl spinor supersymmetries of each chirality. The total number of (real) components of the supersymmetry generators equals $2 n_{+}+2 n_{-}$. If the symplectic-Majorana condition is imposed in each chirality sector, both integers $n_{ \pm}$must be even. The corresponding R-symmetry is $\operatorname{USp}\left(n_{+}\right) \times \operatorname{USp}\left(n_{-}\right)$, under which the supersymmetries transform in the representation $\left(n_{+}, 1\right) \oplus\left(1, n_{-}\right)$. The maximal number of (real) components of the supersymmetry is 32 , while the minimum number is 4 . Many, but not all, of the corresponding supergravity theories may be obtained by dimensional reduction from eleven-dimensional M-theory, or from the ten-dimensional Type IIA, Type IIB, or $\mathcal{N}=1$ (heterotic) supergravities. Below, we provide a list of supergravity theories for which all supersymmetries are subject to the symplectic Majorana condition,

- 32 supersymmetries: The theory with $\left(n_{+}, n_{-}\right)=(4,4)$ is non-chiral; it may be obtained by compactification of Type II (A or B) supergravity on $T^{4}$. Its field content consists of the metric, 8 Rarita-Schwinger fields, 5 anti-symmetric tensor fields, 16 vector fields, 40 spinor fields, and 25 scalars parameterizing a $S O(5,5) /(S O(5) \times S O(5))$ coset. Its Lagrangian and supersymmetry transformations were constructed in [43].

- 32 supersymmetries: The maximally chiral $(0,8)$ theory has been studied in [44], where its field multiplet structure was determined (at the linearized level). To date, full field equations and Lagrangians for the $(0,8)$ and $(2,6)$ theories have not been written down.

- 16 supersymmetries: The Type 4a theory with $\left(n_{+}, n_{-}\right)=(2,2)$ can be obtained by compactification of Type IIA theory on $K 3$ [45]. Its field content consists of the metric, 4 Rarita-Schwinger fields, vector fields, spinor fields, and scalars.

- 16 supersymmetries: The Type 4b theory with $\left(n_{+}, n_{-}\right)=(0,4)$, can be obtained by compactification of Type IIB theory on $K 3$ [40] (see also [41, 42]). This is the theory of central interest to us, and its properties will be given in detail below.

- 8 supersymmetries: The $(0,2)$-theories have been discussed in detail in [42]. 


\subsection{The $(0,4)$ theory}

The six-dimensional Type $4 \mathrm{~b}$ supergravity with $\left(n_{+}, n_{-}\right)=(0,4)$ is a close analogue of tendimensional Type IIB supergravity. The field content of the theory consists of a supergravity multiplet, and $m$ anti-symmetric tensor multiplets. The supergravity multiplet contains the metric ${ }^{5} g_{\mu \nu}$, four negative chirality Rarita-Schwinger fields $\psi_{\mu}^{\alpha}$, and five anti-symmetric tensor fields $B_{\mu \nu}^{I}$ with self-dual field strength. The $m$ anti-symmetric tensor multiplets account for $m$ anti-symmetric tensor fields $B_{\mu \nu}^{R}$ with anti-self-dual field strength, $4 m$ Weyl spinors $\chi^{r \alpha}$ of positive chirality, and $5 m$ scalars $V^{i}{ }_{R}$, which parametrize the coset $S O(5, m) /(S O(5) \times$ $S O(m)$ ). The multiplet structure is summarized in Table 1 below,

\begin{tabular}{|c||c|c|c|c||c|c|}
\hline Field & $g_{\mu \nu}$ & $B_{\mu \nu}^{I}$ & $B_{\mu \nu}^{R}$ & $V^{i} R$ & $\psi_{\mu}^{\alpha}$ & $\chi^{r \alpha}$ \\
\hline \hline $\mathrm{SO}(5)$ & $\mathbf{1}$ & $\mathbf{5}$ & $\mathbf{1}$ & $\mathbf{5}$ & $\mathbf{4}$ & $\mathbf{4}$ \\
\hline $\mathrm{SO}(m)$ & $\mathbf{1}$ & $\mathbf{1}$ & $\mathbf{m}$ & $\mathbf{m}$ & $\mathbf{1}$ & $\mathbf{m}$ \\
\hline
\end{tabular}

Table 1: Field content of six-dimensional $(0,4)$ supergravity

\subsubsection{Chiral truncation of the $(4,4)$ theory}

Consider now the following chiral truncation of the $(4,4)$ theory. We set to zero the sixteen vector fields, the four positive chirality components of the Rarita-Schwinger fields and of the supersymmetry parameters, as well as the twenty negative chirality components of the spinor fields. The field contents, Lagrangian, and supersymmetry transformations of this chiral restriction precisely coincide with those of the $(0,4)$ theory for the special choice of $m=5$. Note that the 5 anti-symmetric tensor fields of the $(4,4)$ theory split into self-dual and anti-self-dual, thereby providing the appropriate fields required for both the supergravity and anti-symmetric tensor multiplets of the $(0,4)$ theory.

It follows immediately that every classical solution to the field equations of the $(0,4)$ theory for $m \leq 5$ may be lifted to a solution of the $(4,4)$ theory with the same number of residual supersymmetries. On the other hand, cancellation of fermion anomalies in the $(0,4)$ theory by itself requires $m=21$, in which case the theory can be obtained by compactification of ten-dimensional Type IIB string theory on $K 3$. In our search for classical (BPS) solutions in the $(0,4)$ theory, we shall keep $m$ arbitrary as long as possible.

\footnotetext{
${ }^{5}$ A detailed summary of notations and conventions for indices may be found in Appendix A.
} 


\subsubsection{Possible truncations of the $(0,8)$ and $(2,6)$ theories ?}

General considerations of supersymmetry representation theory indicate that field theories should exist with $(0,8)$ and $(2,6)$ supersymmetry (totalling 32 supersymmetries). For the $(0,8)$ theory, the field multiplet at the linearized level is known [44], but its extension to the fully interacting level is not known. Even at the linearized level, this theory is clearly out of the ordinary, as the customary metric field for the graviton is replaced here by a rank-four tensor with the symmetries of the Riemann tensor. Even less is known for the $(2,6)$ theory. Yet, both theories are expected to reduce to $N=8$ supergravity in five and in four dimensions, which makes them interesting, at least in principle. Can one expect the $(0,4)$ theory to be a truncation of the $(0,8)$ and $(2,6)$ ? If so, the solutions obtained for the $(0,4)$ theory in this paper would lift also to solutions of the $(0,8)$ and $(2,6)$ theories.

\subsection{Scalar fields}

We begin by spelling out the coset parametrization of the scalar fields, and the duality restrictions on the anti-symmetric tensor fields for $(0,4)$ supergravity [40] (see also [42, 41]) in the subsequent section. Consistently with the discussions of the preceding section, we shall keep the number $m$ of anti-symmetric tensor multiplets arbitrary.

The scalar fields take values in the coset space $S O(5, m) /(S O(5) \times S O(m))$, and may be parametrized in terms of a frame field $V$ which takes values in $S O(5, m)$. A convenient explicit representation may be obtained by block-decomposing the $S O(5, m)$-invariant metric $\eta$ and the frame $V$ according to the maximal compact subgroups $S O(5) \times S O(m)$,

$$
\eta=\left(\begin{array}{cc}
I_{5} & 0 \\
0 & -I_{m}
\end{array}\right) \quad V=V^{(i, r)}{ }_{A}=\left(\begin{array}{cc}
V^{i}{ }_{I} & V^{i}{ }_{R} \\
V^{r}{ }_{I} & V^{r}{ }_{R}
\end{array}\right)
$$

The index $A=(I, R)$ will label the fundamental representation of $S O(5, m)$, while the indices $(i, r)$ will label the fundamental representation of $S O(5) \times S O(m)$, with $I, i=1, \cdots, 5$ and $R, r=6, \cdots, m+5$. Since the indices $i$ and $r$ label different objects, we shall refrain from assembling them into a single index, following [40]. The $S O(5, m)$ index $A$ is raised, lowered, and contracted with the $S O(5, m)$-invariant metric $\eta$, while we will raise, lower, and contract indices $i$ and $r$ with the positive Euclidean metrics $\delta_{i j}$ and $\delta_{r s}$. Since $V \in S O(5, m)$, it obeys,

$$
\eta=V^{t} \eta V \quad V^{-1}=\eta V^{t} \eta
$$

The block decomposition of the right-invariant flat $S O(5, m)$-connection is given by,

$$
\partial_{\mu} V V^{-1}=\left(\begin{array}{cc}
Q_{\mu} & \sqrt{2} P_{\mu} \\
\sqrt{2} R_{\mu} & S_{\mu}
\end{array}\right)
$$


Since $\partial_{\mu} V V^{-1}$ takes values in the Lie algebra of $S O(5, m)$, the product $\partial_{\mu} V V^{-1} \eta$ is antisymmetric, which implies the following relations between the connection components,

$$
Q_{\mu}^{t}=-Q_{\mu} \quad R_{\mu}=P_{\mu}^{t} \quad S_{\mu}^{t}=-S_{\mu}
$$

Here, $Q_{\mu}$ and $S_{\mu}$ are canonical connections taking values in the Lie algebras of $S O(5)$ and $S O(m)$ respectively, while $P_{\mu}$ is a canonical frame on $S O(5, m) /(S O(5) \times S O(m))$.

We represent the coset $S O(5, m) /(S O(5) \times S O(m))$ by a $5 m$-dimensional slice in $S O(5, m)$. We follow the conventions introduced in [40], which are the natural ones associated with the right-invariant connection $\partial_{\mu} V V^{-1}$. A transformation under the action of $\mathcal{M} \in S O(5, m)$ of a point $V$ on the slice to another point $V^{\prime}$ on the slice is by right-multiplication, and must be compensated by a gauge transformation $M \in S O(5) \times S O(m)$ acting on the left,

$$
V \mathcal{M}=M^{-1} V^{\prime} \quad M=\left(\begin{array}{cc}
M_{5} & 0 \\
0 & M_{m}
\end{array}\right)
$$

The canonical frame and connections then transform as follows,

$$
\partial_{\mu} V^{\prime}\left(V^{\prime}\right)^{-1}=M \partial_{\mu} V V^{-1} M^{-1}+\partial_{\mu} M M^{-1}
$$

The component formulas for the canonical frame and connection fields are,

$$
\begin{aligned}
\sqrt{2} P_{\mu}^{i r} & =-\left(\partial_{\mu} V_{A}^{i}\right) V_{B}^{r} \eta^{A B} \\
Q_{\mu}^{i j} & =+\left(\partial_{\mu} V_{A}^{i}\right) V^{j}{ }_{B} \eta^{A B} \\
S_{\mu}^{r s} & =-\left(\partial_{\mu} V^{r}{ }_{A}\right) V^{s}{ }_{B} \eta^{A B}
\end{aligned}
$$

A good parametrization of the coset $S O(5, m) /(S O(5) \times S O(m))$ is provided by $V^{i}$.

\subsection{Anti-symmetric tensor fields}

The anti-symmetric rank-two tensor fields $B_{\mu \nu}^{A}$, respectively $B_{\mu \nu}^{I}$ of the supergravity multiplet, and $B_{\mu \nu}^{R}$ of the anti-symmetric tensor multiplet, give rise to field strength three-forms,

$$
G^{A}=d B^{A}
$$

The associated $S O(5, m)$-covariant field strength three-forms $H^{i}$ and $H^{r}$, defined by,

$$
\begin{aligned}
& H^{i}=V^{i}{ }_{A} G^{A} \\
& H^{r}=V^{r}{ }_{A} G^{A}
\end{aligned}
$$

are respectively self-dual and anti-self-dual three-forms, obeying,

$$
\begin{aligned}
* H^{i} & =+H^{i} \\
* H^{r} & =-H^{r}
\end{aligned}
$$




\subsection{Bianchi identities}

As a result of their definition in (2.3), the fields $P, Q$ and $S$ obey the following Bianchi identities, expressed here in exterior form notation,

$$
\begin{aligned}
d P^{i r}-Q^{i j} \wedge P^{j r}-S^{r s} \wedge P^{i s} & =0 \\
d Q^{i j}-Q^{i k} \wedge Q^{k j}-2 P^{i r} \wedge P^{j r} & =0 \\
d S^{r s}-S^{r t} \wedge S^{t s}-2 P^{i r} \wedge P^{i s} & =0
\end{aligned}
$$

where summation over repeated indices is implied. Similarly, the three-forms $G$ obey simple Bianchi identities,

$$
d G^{A}=0
$$

As a result of the relation (2.9), the fields $H^{i}$ and $H^{r}$ then obey the Bianchi identities,

$$
\begin{aligned}
& d H^{i}-Q^{i j} \wedge H^{j}-\sqrt{2} P^{i r} \wedge H^{r}=0 \\
& d H^{r}-S^{r s} \wedge H^{s}-\sqrt{2} P^{i r} \wedge H^{i}=0
\end{aligned}
$$

\subsection{Field equations for bosonic fields}

The Bianchi identities and field equations for the bosonic fields are as follows. For the field strengths $H^{i}$ and $H^{r}$, the Bianchi identities and field equations are equivalent to one another in view of their duality properties. Substituting (2.10) into (2.13), we find the following field equations for $H^{i}, H^{r}$,

$$
\begin{aligned}
& d * H^{i}-Q^{i j} \wedge * H^{j}+\sqrt{2} P^{i r} \wedge * H^{r}=0 \\
& d * H^{r}-S^{r s} \wedge * H^{s}+\sqrt{2} P^{i r} \wedge * H^{i}=0
\end{aligned}
$$

In components these equations read as follows,

$$
\begin{aligned}
& \nabla^{\mu} H_{\mu \nu \rho}^{i}-\left(Q^{\mu}\right)^{i j} H_{\mu \nu \rho}^{j}+\sqrt{2}\left(P^{\mu}\right)^{i r} H_{\mu \nu \rho}^{r}=0 \\
& \nabla^{\mu} H_{\mu \nu \rho}^{r}-\left(S^{\mu}\right)^{r s} H_{\mu \nu \rho}^{s}+\sqrt{2}\left(P^{\mu}\right)^{i r} H_{\mu \nu \rho}^{i}=0
\end{aligned}
$$

where $\nabla^{\mu}$ is the covariant derivative with respect to the affine connection. The Einstein equations are given by,

$$
R_{\mu \nu}-H_{\mu \rho \sigma}^{i} H_{\nu}^{i \rho \sigma}-H_{\mu \rho \sigma}^{r} H_{\nu}^{r \rho \sigma}-2 P_{\mu}^{i r} P_{\nu}^{i r}=0
$$

The field equation for the scalars is given by,

$$
\nabla^{\mu} P_{\mu}^{i r}-\left(Q^{\mu}\right)^{i j} P_{\mu}^{j r}-\left(S^{\mu}\right)^{r s} P_{\mu}^{i s}-\frac{\sqrt{2}}{3} H^{i \mu \nu \rho} H_{\mu \nu \rho}^{r}=0
$$




\subsection{Supersymmetry transformation for fermionic fields}

The fermionic fields $\psi_{\mu}^{\alpha}$ and $\chi^{r \alpha}$ of the $(0,4)$ supergravity, as well as the local supersymmetry spinor parameter $\varepsilon^{\alpha}$, have definite chiralities, and obey the symplectic Majorana conditions, given by,

$$
\begin{aligned}
\gamma^{7} \psi_{\mu}^{\alpha} & =-\psi_{\mu}^{\alpha} & \psi_{\mu}^{\alpha} & =\mathcal{B C}_{\beta}^{\alpha}\left(\psi_{\mu}^{\beta}\right)^{*} \\
\gamma^{7} \chi^{r \alpha} & =+\chi^{r \alpha} & \chi^{r \alpha} & =\mathcal{B} \mathcal{C}_{\beta}^{\alpha}\left(\chi^{r \beta}\right)^{*} \\
\gamma^{7} \varepsilon^{\alpha} & =-\varepsilon^{\alpha} & \varepsilon^{\alpha} & =\mathcal{B} \mathcal{C}_{\beta}^{\alpha}\left(\varepsilon^{\beta}\right)^{*}
\end{aligned}
$$

Representations for Dirac matrices for $S O(1,5)$ and $S O(5)$ may be found in Appendix A, where a number of useful Dirac algebra relations have also been collected. In particular, $\mathcal{B}$ is the $S O(1,5)$ complex conjugation matrix, while $\mathcal{C}$ is the $S O(5)$ charge conjugation matrix, whose definitions and representations are given in (A.10) and (A.16) of Appendix A.

The supersymmetry transformations on the fermionic fields are given by, ${ }^{6}$

$$
\begin{aligned}
\delta \psi_{\mu}^{\alpha} & =D_{\mu} \varepsilon^{\alpha}-\frac{1}{4} H_{\mu \nu \rho}^{i} \gamma^{\nu \rho}\left(\Gamma^{i}\right)_{\beta}^{\alpha} \varepsilon^{\beta} \\
\delta \chi^{r \alpha} & =\frac{1}{\sqrt{2}} \gamma^{\mu} P_{\mu}^{i r}\left(\Gamma^{i}\right)_{\beta}^{\alpha} \varepsilon^{\beta}+\frac{1}{12} \gamma^{\mu \nu \rho} H_{\mu \nu \rho}^{r} \varepsilon^{\alpha}
\end{aligned}
$$

The covariant derivative in $(2.18)$ is taken with respect to the $S O(1,5)$ spin connection $\omega_{\mu}$ and the $S O(5)$-connection $Q_{\mu}$, and given by,

$$
D_{\mu} \varepsilon^{\alpha} \equiv \partial_{\mu} \varepsilon^{\alpha}+\frac{1}{4} \omega_{\mu}^{M N} \gamma_{M N} \varepsilon^{\alpha}-\frac{1}{4} Q_{\mu}^{i j}\left(\Gamma^{i j}\right)^{\alpha}{ }_{\beta} \varepsilon^{\beta}
$$

It is manifest that these relations are consistent with the chirality restrictions of (2.18). They are also consistent with the symplectic Majorana condition, as may be established by analyzing the complex conjugate relations. Finally, using the chirality of $\varepsilon^{\alpha}$, as well as formula (A.9), we derive the relation, $\gamma^{\mu \nu \rho} H_{\mu \nu \rho}^{r} \varepsilon^{\alpha}=-\gamma^{\mu \nu \rho}\left(* H^{r}\right)_{\mu \nu \rho} \varepsilon^{\alpha}$. Therefore, the last term in $\delta \chi^{r \alpha}$ is non-zero only if $* H^{r}=-H^{r}$, which is indeed the case from $(2.10)$.

The BPS equations correspond to the vanishing of the supersymmetry transformations,

$$
\delta \psi_{\mu}^{\alpha}=\delta \chi^{r \alpha}=0
$$

for all fermionic fields, while setting the fermionic fields to zero. For non-trivial $\varepsilon^{\alpha}$, the corresponding bosonic solutions are invariant under some degree of supersymmetry.

\footnotetext{
${ }^{6}$ The Dirac matrices $\gamma^{\mu}$ with respect to coordinate indices are related to the Dirac matrices $\gamma^{M}$ with respect to frame indices by $\gamma^{M}=e^{M}{ }_{\mu} \gamma^{\mu}$.
} 


\section{Ansatz and reduced BPS equations}

In this section, we construct the general Ansatz invariant under $S O(2,1) \times S O(3)$ for the bosonic fields and supersymmetry transformation parameters of $(0,4)$ supergravity. The BPS equations of (2.21) will then be reduced by restricting them to this Ansatz.

\subsection{The $S O(2,1) \times S O(3)$-invariant Ansatz}

We seek the most general Ansatz in six-dimensional $(0,4)$ supergravity with $S O(2,1) \times S O(3)$ symmetry. The $S O(2,1)$ requires the geometry to contain a factor of $A d S_{2}$, while the $S O(3)$ requires a factor of $S^{2}$, both of which are warped over a two-dimensional surface $\Sigma$, yielding a total warped space-time structure $A d S_{2} \times S^{2} \times \Sigma$. The Ansatz for the metric is given by,

$$
d s^{2}=f_{1}^{2} d s_{A d S_{2}}^{2}+f_{2}^{2} d s_{S^{2}}^{2}+d s_{\Sigma}^{2}
$$

where $f_{1}$ and $f_{2}$ are real functions on $\Sigma$. Orthonormal frames are defined by,

$$
\begin{aligned}
& d s_{A d S_{2}}^{2}=\eta_{m n}^{(2)} \hat{e}^{m} \otimes \hat{e}^{n} \quad e^{m}=f_{1} \hat{e}^{m} \quad m=0,1 \\
& d s_{S^{2}}^{2}=\delta_{p q} \hat{e}^{p} \otimes \hat{e}^{q} \quad e^{p}=f_{2} \hat{e}^{p} \quad p=2,3 \\
& d s_{\Sigma}^{2}=\delta_{a b} e^{a} \otimes e^{b} \quad e^{a}=\rho \hat{e}^{a} \quad a=4,5
\end{aligned}
$$

The metrics $d s_{A d S_{2}}^{2}$ and $d s_{S^{2}}^{2}$, as well as the orthonormal frames $\hat{e}^{m}$ and $\hat{e}^{p}$, respectively refer to the spaces $A d S_{2}$ and $S^{2}$ with unit radius. The Ansatz for the scalar field strength one-forms $P^{i r}$, as well as the composite connection one-forms $Q^{i j}$ and $S^{r s}$ is as follows,

$$
\begin{aligned}
P^{i r} & =p_{a}^{i r} e^{a} \\
Q^{i j} & =q_{a}^{i j} e^{a} \\
S^{r s} & =s_{a}^{r s} e^{a}
\end{aligned}
$$

Finally, the Ansatz for the three form field strength is,

$$
\begin{aligned}
& H^{i}=g_{a}^{i} e^{01 a}+h_{a}^{i} e^{23 a} \\
& H^{r}=\tilde{g}_{a}^{r} e^{01 a}+\tilde{h}_{a}^{r} e^{23 a}
\end{aligned}
$$

The functions $f_{1}, f_{2}, \rho, p_{a}^{i r}, q_{a}^{i j}, s_{a}^{r s}, g_{a}^{i}, h_{a}^{i}, \tilde{g}_{a}^{r}, \tilde{h}_{a}^{r}$ depend only on the coordinates on $\Sigma$. The duality relations (2.10), combined with $* e^{01 a}=\epsilon^{a}{ }_{b} e^{23 b}$ and $* e^{23 a}=-\epsilon^{a}{ }_{b} e^{01 b}$, produce the following relations,

$$
\begin{aligned}
& h_{a}^{i}=-\epsilon_{a}{ }^{b} g_{b}^{i} \\
& \tilde{h}_{a}^{r}=+\epsilon_{a}{ }^{b} \tilde{g}_{b}^{r}
\end{aligned}
$$




\subsection{Killing spinor basis}

A convenient basis of Killing spinors on $A d S_{2} \times S^{2} \times \Sigma$ is provided by the spinors $\chi^{\eta_{1}, \eta_{2}, \eta_{3}}$ of $S O(2,1) \times S O(3) \times S O(2)$, which are defined by the following equations,

$$
\begin{array}{rlr}
\left(\hat{\nabla}_{m}-\frac{1}{2} \eta_{1} \tilde{\gamma}_{m} \otimes I \otimes I\right) \chi^{\eta_{1}, \eta_{2}, \eta_{3}} & =0 & m=0,1 \\
\left(\hat{\nabla}_{p}-\frac{i}{2} \eta_{2} I \otimes \tilde{\gamma}_{p} \otimes I\right) \chi^{\eta_{1}, \eta_{2}, \eta_{3}} & =0 & p=2,3 \\
I \otimes I \otimes\left(I-\eta_{3} \sigma^{3}\right) \chi^{\eta_{1}, \eta_{2}, \eta_{3}} & =0 &
\end{array}
$$

Integrability requires $\eta_{1}^{2}=\eta_{2}^{2}=\eta_{3}^{2}=1$. Given $\eta$, there are 4 independent solutions $\chi^{\eta_{1}, \eta_{2}, \eta_{3}}$ to (3.6). An explicit representation for the Dirac matrices $\tilde{\gamma}_{m}$ and $\tilde{\gamma}_{p}$ on $A d S_{2}$ and $S^{2}$ respectively is given in Appendix A. The chirality matrices on $A d S_{2} \times S^{2}$ reverse the signs of the $\eta_{1}$ and $\eta_{2}$, allowing us to make the following identifications,

$$
\begin{aligned}
& \left(\sigma^{3} \otimes I \otimes I\right) \chi^{\eta_{1}, \eta_{2}, \eta_{3}}=\chi^{-\eta_{1}, \eta_{2}, \eta_{3}} \\
& \left(I \otimes \sigma^{3} \otimes I\right) \chi^{\eta_{1}, \eta_{2}, \eta_{3}}=\chi^{\eta_{1},-\eta_{2}, \eta_{3}}
\end{aligned}
$$

Under charge conjugation of $\chi$, we produce a spinor $\chi^{c}$, defined by,

$$
\left(\chi^{c}\right)^{\eta_{1}, \eta_{2}, \eta_{3}}=\mathcal{B}\left(\chi^{\eta_{1}, \eta_{2}, \eta_{3}}\right)^{*}
$$

Since six-dimensional Minkowski space-time does not support Majorana spinors, $\chi^{c}$ can never be identified with $\chi$, and therefore produces an independent spinor. It will be convenient to chose the space of $\chi$ and $\chi^{c}$ spinors as a basis for the full 8-components supersymmetry spinors $\varepsilon^{\alpha}$, which gives us the following decomposition,

$$
\varepsilon^{\alpha}=\sum_{\eta_{1}, \eta_{2}, \eta_{3}}\left(\chi^{\eta_{1}, \eta_{2}, \eta_{3}} \otimes \zeta_{\eta_{1}, \eta_{2}, \eta_{3}}^{\alpha}+\left(\chi^{c}\right)^{\eta_{1}, \eta_{2}, \eta_{3}} \otimes \hat{\zeta}_{\eta_{1}, \eta_{2}, \eta_{3}}^{\alpha}\right)
$$

where the coefficients $\zeta_{\eta_{1}, \eta_{2}, \eta_{3}}^{\alpha}$ and $\hat{\zeta}_{\eta_{1}, \eta_{2}, \eta_{3}}^{\alpha}$ are spinors of $S O(5)$, as indicated by the index $\alpha$. As explained in Appendix A, there is an additional two-fold degeneracy of $\chi^{\eta_{1}, \eta_{2}, \eta_{3}}$ which will double the number of supersymmetries.

The six-dimensional chirality matrix $\gamma^{7}$, which in our basis is given by $\gamma^{7}=\sigma^{3} \otimes \sigma^{3} \otimes \sigma^{3}$, relates different components of $\chi$ and $\chi^{c}$,

$$
\begin{aligned}
\gamma^{7} \chi^{\eta_{1}, \eta_{2}, \eta_{3}} & =\eta_{3} \chi^{-\eta_{1},-\eta_{2}, \eta_{3}} \\
\gamma^{7}\left(\chi^{c}\right)^{\eta_{1}, \eta_{2}, \eta_{3}} & =\eta_{3}\left(\chi^{c}\right)^{-\eta_{1},-\eta_{2}, \eta_{3}}
\end{aligned}
$$


As a result, the negative chirality condition $\gamma^{7} \varepsilon^{\alpha}=-\varepsilon^{\alpha}$ imposes the following relations between the different components of $\zeta$, and between the different components of $\hat{\zeta}$,

$$
\begin{aligned}
& \zeta_{\eta_{1}, \eta_{2}, \eta_{3}}^{\alpha}=-\eta_{3} \zeta_{-\eta_{1},-\eta_{2}, \eta_{3}}^{\alpha} \\
& \hat{\zeta}_{\eta_{1}, \eta_{2}, \eta_{3}}^{\alpha}=-\eta_{3} \hat{\zeta}_{-\eta_{1},-\eta_{2}, \eta_{3}}^{\alpha}
\end{aligned}
$$

The symplectic Majorana condition $\varepsilon^{\alpha}=\mathcal{B C}^{\alpha}{ }_{\beta}\left(\varepsilon^{\beta}\right)^{*}$ imposes relations between $\zeta$ and $\hat{\zeta}$,

$$
\begin{aligned}
& \zeta_{\eta_{1}, \eta_{2}, \eta_{3}}^{\alpha}=-\mathcal{C}^{\alpha}{ }_{\beta}\left(\hat{\zeta}_{\eta_{1}, \eta_{2}, \eta_{3}}^{\beta}\right)^{*} \\
& \hat{\zeta}_{\eta_{1}, \eta_{2}, \eta_{3}}^{\alpha}=+\mathcal{C}^{\alpha}{ }_{\beta}\left(\zeta_{\eta_{1}, \eta_{2}, \eta_{3}}^{\beta}\right)^{*}
\end{aligned}
$$

where $\mathcal{C}$ is the charge conjugation matrix of $S O(5)$, given in our basis by $\mathcal{C}=\sigma^{1} \otimes \sigma^{2}$. As a

result, $\hat{\zeta}$ may be eliminated in terms of $\zeta^{*}$, yielding our final decomposition formula for the supersymmetry spinor,

$$
\varepsilon^{\alpha}=\sum_{\eta_{1}, \eta_{2}, \eta_{3}}\left(\chi^{\eta_{1}, \eta_{2}, \eta_{3}} \otimes \zeta_{\eta_{1}, \eta_{2}, \eta_{3}}^{\alpha}+\left(\chi^{c}\right)^{\eta_{1}, \eta_{2}, \eta_{3}} \otimes \mathcal{C}_{\beta}^{\alpha} \zeta_{\eta_{1}, \eta_{2}, \eta_{3}}^{\beta *}\right)
$$

Since the BPS equations are compatible with the symplectic Majorana condition, the reduced BPS equations for $\zeta$ and $\zeta^{*}$ will automatically be complex conjugates of one another.

\subsection{Derivation of the reduced BPS equations}

The detailed derivation of the reduced BPS equations will be presented in Appendix B. Here, we shall provide only a summary of the results. We use the standard notation for the Pauli matrices $\tau^{a}$, with $a=0,1,2,3$, acting on the indices $\eta$ of $\zeta$,

$$
\left(\tau^{(a b c)} \zeta^{\alpha}\right)_{\eta_{1}, \eta_{2}, \eta_{3}} \equiv \sum_{\eta_{1}^{\prime}, \eta_{2}^{\prime}, \eta_{3}^{\prime}}\left(\tau^{a}\right)_{\eta_{1}, \eta_{1}^{\prime}}\left(\tau^{b}\right)_{\eta_{2}, \eta_{2}^{\prime}}\left(\tau^{c}\right)_{\eta_{3}, \eta_{3}^{\prime}} \zeta_{\eta_{1}^{\prime}, \eta_{2}^{\prime}, \eta_{3}^{\prime}}^{\alpha}
$$

In this notation, the chirality condition becomes,

$$
\tau^{(113)} \zeta=-\zeta
$$

The reduced BPS equations for the variation of the gravitini field are,

$$
\begin{aligned}
(m) \quad 0 & =\frac{1}{f_{1}} \tau^{(300)} \zeta+\frac{D_{a} f_{1}}{f_{1}} \tau^{(11 a)} \zeta+g_{a}^{i} \tau^{(01 a)} \Gamma_{i} \zeta \\
(p) \quad 0 & =\frac{i}{f_{2}} \tau^{(130)} \zeta+\frac{D_{a} f_{2}}{f_{2}} \tau^{(11 a)} \zeta-i h_{a}^{i} \tau^{(10 a)} \Gamma_{i} \zeta \\
(a) \quad 0 & =\left(D_{a}+\frac{i}{2} \hat{\omega}_{a} \tau^{(003)}-\frac{1}{4} q_{a}^{i j} \Gamma_{i j}\right) \zeta+\frac{1}{2} g_{a}^{i} \tau^{(100)} \Gamma_{i} \zeta-\frac{i}{2} h_{a}^{i} \tau^{(010)} \Gamma_{i} \zeta
\end{aligned}
$$


while the reduced BPS equations for the fermions $\chi^{r \alpha}$ are given by,

$$
\text { (c) } 0=\sqrt{2} p_{a}^{i r} \tau^{(11 a)} \Gamma_{i} \zeta-\tilde{g}_{a}^{r} \tau^{(01 a)} \zeta+i \tilde{h}_{a}^{r} \tau^{(10 a)} \zeta
$$

Next, we perform the substitution $\zeta=-\tau^{(113)} \zeta$ in the second term of equation $(\mathrm{m})$, in the fourth term of equation (a), and in the second term of equation (c), and then multiply equations (p) and (c) to the left by $\tau^{(100)}$. We see that the two eigenmodes of $\tau^{(300)}$ decouple from one another and are mapped into one another by reversing the sign of $f_{1}$. Given one sign for the physical field $f_{1}$, one or the other eigenmode must vanish. We choose the nonvanishing mode to be the one corresponding to eigenvalue +1 , and omit the first index entry in the $\tau$-matrices, so that $\tau^{(0 b c)} \rightarrow \tau^{(b c)}$. In the same vein, we continue to use the notation $\zeta$ for the eigenmode of $\tau^{(300)}$ with eigenvalue +1 . Finally, we perform a cyclic permutation on the Pauli matrices,

$$
\tau^{(10)} \rightarrow \tau^{(20)}, \quad \tau^{(20)} \rightarrow \tau^{(30)}, \quad \tau^{(30)} \rightarrow \tau^{(10)}
$$

After multiplying the resulting equation (p') on the left by $\tau^{(13)}$, and eliminating $h_{a}^{i}$ and $\tilde{h}_{a}^{r}$ in terms of respectively $g_{a}^{i}$ and $\tilde{g}_{a}^{r}$ using the duality equations (3.5), the equations become,

$$
\begin{aligned}
\left(m^{\prime \prime}\right) \quad 0 & =\frac{1}{f_{1}} \zeta-\frac{D_{a} f_{1}}{f_{1}} \tau^{(0 a)} \tau^{(03)} \zeta+g_{a}^{i} \tau^{(2 a)} \Gamma_{i} \zeta \\
\left(p^{\prime \prime}\right) \quad 0 & =\frac{1}{f_{2}} \tau^{(33)} \zeta-\frac{D_{a} f_{2}}{f_{2}} \tau^{(0 a)} \tau^{(03)} \zeta-g_{a}^{i} \tau^{(2 a)} \Gamma_{i} \zeta \\
\left(a^{\prime \prime}\right) \quad 0 & =\left(D_{a}+\frac{i}{2} \hat{\omega}_{a} \tau^{(03)}\right) \zeta-\frac{1}{4} q_{a}^{i j} \Gamma_{i j} \zeta-\frac{1}{2} g_{a}^{i} \tau^{(23)} \Gamma_{i} \zeta+\frac{i}{2} \epsilon_{a b} g_{b}^{i} \tau^{(20)} \Gamma_{i} \zeta \\
\left(c^{\prime \prime}\right) \quad 0 & =\frac{1}{\sqrt{2}} p_{a}^{i r} \tau^{(0 a)} \tau^{(03)} \Gamma_{i} \zeta+\tilde{g}_{a}^{r} \tau^{(2 a)} \zeta
\end{aligned}
$$

\subsection{Chiral form of the reduced BPS equations}

It will be convenient to further reduce the representation of the spinors, by reformulating the frame $e^{a}$ with $a=4,5$ on the Riemann surface $\Sigma$ in terms of a complex basis, $e^{a}=\left(e^{z}, e^{\bar{z}}\right)$, with metric and $\epsilon$-tensor normalized by,

$$
\delta_{z \bar{z}}=\delta_{\bar{z} z}=2 \quad \epsilon_{z \bar{z}}=-\epsilon_{\bar{z} z}=2 i
$$

and as given by the following explicit formulas,

$$
\begin{aligned}
e^{z} & =\left(e^{4}+i e^{5}\right) / 2 & e_{z} & =e^{4}-i e^{5} \\
e^{\bar{z}} & =\left(e^{4}-i e^{5}\right) / 2 & e_{\bar{z}} & =e^{4}+i e^{5}
\end{aligned}
$$


Using these conventions the metric factor on $\Sigma$ is given by

$$
d s_{\Sigma}^{2}=4 \rho^{2}|d w|^{2}
$$

Corresponding relations hold for the the fields $p_{a}^{i r}, q_{a}^{i j}, g_{a}^{i}$, so that, for example, $p_{z}^{i r}=p_{4}^{i r}-i p_{5}^{i r}$, and $p_{\bar{z}}^{i r}=p_{4}^{i r}+i p_{5}^{i r}$. The Pauli matrices in this basis take the form,

$$
\sigma^{z}=\tau^{z}=\left(\begin{array}{ll}
0 & 1 \\
0 & 0
\end{array}\right) \quad \sigma^{\bar{z}}=\tau^{\bar{z}}=\left(\begin{array}{ll}
0 & 0 \\
1 & 0
\end{array}\right)
$$

We decompose $\zeta^{\alpha}$ into its two components of the $\eta_{3}$ index, leaving the $\eta_{2}$ index free,

$$
\xi_{\eta_{2}}=\zeta_{\eta_{2},+} \quad \psi_{\eta_{2}}=\zeta_{\eta_{2},-}
$$

The BPS equations in the chiral form may be grouped into algebraic equations in $\psi, \xi$,

$$
\begin{aligned}
& \left(m_{1}\right) \quad 0=\frac{1}{f_{1}} \xi+\frac{D_{z} f_{1}}{f_{1}} \psi+g_{z}^{i} \tau^{(2)} \Gamma_{i} \psi \\
& \left(m_{2}\right) \quad 0=\frac{1}{f_{1}} \psi-\frac{D_{\bar{z}} f_{1}}{f_{1}} \xi+g_{\bar{z}}^{i} \tau^{(2)} \Gamma_{i} \xi \\
& \left(p_{1}\right) \quad 0=\frac{1}{f_{2}} \tau^{(3)} \xi+\frac{D_{z} f_{2}}{f_{2}} \psi-g_{z}^{i} \tau^{(2)} \Gamma_{i} \psi \\
& \left(p_{2}\right) \quad 0=\frac{1}{f_{2}} \tau^{(3)} \psi+\frac{D_{\bar{z}} f_{2}}{f_{2}} \xi+g_{\bar{z}}^{i} \tau^{(2)} \Gamma_{i} \xi \\
& \left(c_{1}\right) \quad 0=-\frac{1}{\sqrt{2}} p_{z}^{i r} \Gamma_{i} \psi+\tilde{g}_{z}^{r} \tau^{(2)} \psi \\
& \left(c_{2}\right) \quad 0=\frac{1}{\sqrt{2}} p_{\bar{z}}^{i r} \Gamma_{i} \xi+\tilde{g}_{\bar{z}}^{r} \tau^{(2)} \xi
\end{aligned}
$$

and differential equations in $\psi, \xi$,

$$
\begin{array}{ll}
\left(a z_{1}\right) & 0=\left(D_{z}+\frac{i}{2} \hat{\omega}_{z}\right) \xi-\frac{1}{4} q_{z}^{i j} \Gamma_{i j} \xi-g_{z}^{i} \tau^{(2)} \Gamma_{i} \xi \\
\left(a z_{2}\right) & 0=\left(D_{z}-\frac{i}{2} \hat{\omega}_{z}\right) \psi-\frac{1}{4} q_{z}^{i j} \Gamma_{i j} \psi \\
\left(a \bar{z}_{1}\right) & 0=\left(D_{\bar{z}}+\frac{i}{2} \hat{\omega}_{\bar{z}}\right) \xi-\frac{1}{4} q_{\bar{z}}^{i j} \Gamma_{i j} \xi \\
\left(a \bar{z}_{2}\right) & 0=\left(D_{\bar{z}}-\frac{i}{2} \hat{\omega}_{\bar{z}}\right) \psi-\frac{1}{4} q_{\bar{z}}^{i j} \Gamma_{i j} \psi+g_{\bar{z}}^{i} \tau^{(2)} \Gamma_{i} \psi
\end{array}
$$

In a system of local complex coordinates $w, \bar{w}$ on $\Sigma$, we have,

$$
\begin{array}{lll}
e^{z}=\rho d w & D_{z}=\rho^{-1} \partial_{w} & \hat{\omega}_{z}=+i \rho^{-2} \partial_{w} \rho \\
e^{\bar{z}}=\rho d \bar{w} & D_{\bar{z}}=\rho^{-1} \partial_{\bar{w}} & \hat{\omega}_{\bar{z}}=-i \rho^{-2} \partial_{\bar{w}} \rho
\end{array}
$$




\section{Solving the reduced BPS equations}

In this section, we present a systematic solution for the BPS equations with 8 supersymmetries. This section is somewhat technical, and the impatient reader may wish to skip it and move directly to Section 5 where a summary of the solution to the BPS equations is given.

Our starting point will be the set of reduced BPS equations in chiral form of (3.25) and (3.26). Viewed as equations on the spinors $\xi$ and $\psi$, equations $\left(m_{1,2}\right),\left(p_{1,2}\right)$, and $\left(c_{1,2}\right)$ are purely algebraic, while $\left(a z_{1,2}\right),\left(a \bar{z}_{1,2}\right)$ are partial differential equations. We shall now solve those in succession.

\subsection{Algebraic relation between $\xi$ and $\psi$}

The combinations $\left(m_{1}\right)+\left(p_{1}\right)$ and $\left(m_{2}\right)+\left(p_{2}\right)$ no longer involve $g_{z}^{i}$ and produce purely algebraic relations between the components of $\xi$ and $\psi$,

$$
\begin{aligned}
& 0=\left(f_{2} \pm f_{1}\right) \xi_{ \pm}+D_{z}\left(f_{1} f_{2}\right) \psi_{ \pm} \\
& 0=\left(f_{2} \mp f_{1}\right) \psi_{ \pm}-D_{\bar{z}}\left(f_{1} f_{2}\right) \xi_{ \pm}
\end{aligned}
$$

Defining the tensor $Y$ of type $(1,0)$ by,

$$
Y \equiv \frac{D_{z}\left(f_{1} f_{2}\right)}{f_{1}+f_{2}}
$$

the $f_{2}+f_{1}$ relations in (4.1) may be recast in the following form,

$$
\xi_{+}=-Y \psi_{+} \quad \xi_{-}=\left(Y^{*}\right)^{-1} \psi_{-}
$$

while the $f_{2}-f_{1}$ relations take the form,

$$
\frac{f_{1}-f_{2}}{f_{1}+f_{2}} \xi_{-}=Y \psi_{-} \quad \frac{f_{1}-f_{2}}{f_{1}+f_{2}} \psi_{+}=-Y^{*} \xi_{+}
$$

Consistency of (4.3) and (4.4) requires that

$$
|Y|^{2}=\frac{f_{1}-f_{2}}{f_{1}+f_{2}}
$$

and

$$
\left|D_{z}\left(f_{1} f_{2}\right)\right|^{2}=f_{1}^{2}-f_{2}^{2}
$$

Condition of (4.6) is equivalent to (4.5) upon use of (4.2). When conditions (4.2), (4.5), and (4.6) are obeyed, the relations of (4.4) follow and may be consistently omitted. This provides the most general solution to equations $\left(m_{1}\right)+\left(p_{1}\right)$ and $\left(m_{2}\right)+\left(p_{2}\right)$. 


\subsection{Identifying the harmonic function $H$}

Next, we shall solve combinations of the differential equations. We begin by eliminating $\xi_{ \pm}$ from the differential equation $\left(a z_{1}\right)$ in (3.26), in favor of $\psi_{ \pm}$and $Y$, using (4.2). The resulting equations are,

$$
\begin{aligned}
0 & =\left(D_{z}+\frac{i}{2} \hat{\omega}_{z}+D_{z} \ln Y\right) \psi_{+}-\frac{1}{4} q_{z}^{i j} \Gamma_{i j} \psi_{+}-\frac{i}{|Y|^{2}} g_{z}^{i} \Gamma_{i} \psi_{-} \\
0 & =\left(D_{z}+\frac{i}{2} \hat{\omega}_{z}-D_{z} \ln Y^{*}\right) \psi_{-}-\frac{1}{4} q_{z}^{i j} \Gamma_{i j} \psi_{-}+i|Y|^{2} g_{z}^{i} \Gamma_{i} \psi_{+}
\end{aligned}
$$

Using equation $\left(a z_{2}\right)$ of (3.26) to eliminate $D_{z} \psi_{ \pm}$we find the algebraic equations,

$$
\begin{aligned}
0 & =\left(i \hat{\omega}_{z}+D_{z} \ln Y\right) \psi_{+}-\frac{i}{|Y|^{2}} g_{z}^{i} \Gamma_{i} \psi_{-} \\
0 & =\left(i \hat{\omega}_{z}-D_{z} \ln Y^{*}\right) \psi_{-}+i|Y|^{2} g_{z}^{i} \Gamma_{i} \psi_{+}
\end{aligned}
$$

Eliminating $i g_{z}^{i} \Gamma^{i} \psi_{ \pm}$between these equations and the corresponding ones in (4.12) gives

$$
\begin{aligned}
& 0=D_{z}\left(f_{1}-f_{2}\right)-\left(f_{1}-f_{2}\right)\left(i \hat{\omega}_{z}+D_{z} \ln Y\right) \\
& 0=D_{z}\left(f_{1}+f_{2}\right)-\left(f_{1}+f_{2}\right)\left(i \hat{\omega}_{z}-D_{z} \ln Y^{*}\right)
\end{aligned}
$$

Using $\hat{\omega}_{z}=i \rho^{-2} \partial_{w} \rho$ and $D_{z}=\rho^{-1} \partial_{w}$, we identify holomorphic one-forms $c_{ \pm}(w)$, given by,

$$
\begin{aligned}
\rho\left(f_{1}-f_{2}\right) / Y^{*} & =c_{-}(w) \\
\rho\left(f_{1}+f_{2}\right) Y & =c_{+}(w)
\end{aligned}
$$

Taking the ratio, and using (4.5), we see that $c_{+}(w)=c_{-}(w)=c(w)$. Eliminating $\xi$ also from equation $\left(a \bar{z}_{1}\right)$ using $(4.2)$, and using equation $\left(a \bar{z}_{2}\right)$ to eliminate $D_{\bar{z}} \psi$ reproduces the same algebraic equations of (4.9), with the same solutions.

Using the defining equation for $Y$ in (4.2), and eliminating the combination $\rho\left(f_{1}+f_{2}\right) Y$ in favor of $c_{+}(w)=c(w)$ using the second equation in (4.10), we find, $\partial_{w}\left(f_{1} f_{2}\right)=c(w)$. Since $f_{1} f_{2}$ is real, this equation may be integrated in terms of a real harmonic function $H$,

$$
\begin{array}{rlrl}
f_{1} f_{2} & =H & c(w)=\partial_{w} H \\
\rho^{2}\left(f_{1}^{2}-f_{2}^{2}\right) & =\left|\partial_{w} H\right|^{2}
\end{array}
$$

where the second equation of (4.11) results from using the second equation in (4.5). These equations can be solved for $f_{1}$ and $f_{2}$ in terms of $\rho$ and $H$. 


\subsection{Algebraic projector conditions}

Next, we consider the remaining combinations, $f_{1}\left(m_{1}\right)-f_{2} \tau^{(2)}\left(p_{1}\right)$ and $f_{1}\left(m_{2}\right)+f_{2} \tau^{(2)}\left(p_{2}\right)$, and eliminate $\xi_{ \pm}$using (4.2) and (4.5), which gives,

$$
\begin{aligned}
& D_{z}\left(f_{1}-f_{2}\right) \psi_{+}=+i g_{z}^{i} \Gamma_{i}\left(f_{1}+f_{2}\right) \psi_{-} \\
& D_{z}\left(f_{1}+f_{2}\right) \psi_{-}=-i g_{z}^{i} \Gamma_{i}\left(f_{1}-f_{2}\right) \psi_{+} \\
& D_{\bar{z}}\left(f_{1}+f_{2}\right) \psi_{+}=+i g_{\bar{z}}^{i} \Gamma_{i}\left(f_{1}+f_{2}\right) \psi_{-} \\
& D_{\bar{z}}\left(f_{1}-f_{2}\right) \psi_{-}=-i g_{\bar{z}}^{i} \Gamma_{i}\left(f_{1}-f_{2}\right) \psi_{+}
\end{aligned}
$$

Any single one of these equations may be retained as giving $\psi_{-}$in terms of $\psi_{+}$or vice-versa. The existence of non-vanishing solutions requires these equations to be compatible with one another. Compatibility of the first group of two equations requires,

$$
\left(f_{1}^{2}-f_{2}^{2}\right) g_{z}^{i} g_{z}^{i}=D_{z}\left(f_{1}-f_{2}\right) D_{z}\left(f_{1}+f_{2}\right)
$$

while compatibility of the second group is given by the complex conjugate of (4.13). Compatibility of the first group with the second group may be expressed as projector equations,

$$
\begin{aligned}
g_{z}^{i} g_{\bar{z}}^{j} \Gamma_{i} \Gamma_{j} \psi_{ \pm} & =N_{\mp}^{2} \psi_{ \pm} \\
g_{\bar{z}}^{i} g_{z}^{j} \Gamma_{i} \Gamma_{j} \psi_{ \pm} & =N_{ \pm}^{2} \psi_{ \pm}
\end{aligned}
$$

where we have defined $N_{ \pm}^{2}$ by,

$$
\left(f_{1}^{2}-f_{2}^{2}\right) N_{ \pm}^{2}=\left|D_{z}\left(f_{1} \pm f_{2}\right)\right|^{2}
$$

Since $g_{z}^{i} g_{\bar{z}}^{j} \Gamma_{i} \Gamma_{j}=\left(g_{z}^{i} \Gamma_{i}\right)\left(g_{z}^{i} \Gamma_{i}\right)^{\dagger}$ we have $N_{ \pm}^{2} \geq 0$, and as a result $f_{1}^{2}>f_{2}^{2}$. Assuming (4.13) the equations in (4.14) are equivalent to one another. Equations (4.2), (4.5), and (4.12) are equivalent to the algebraic equations $\left(m_{1,2}\right)$ and $\left(p_{1,2}\right)$.

\subsection{Solving the projector conditions}

In view of the equivalence of the equations of (4.14), we retain only the first, recast it in terms of the real components of $g_{a}^{i}$ using $g_{4}^{i}-i g_{5}^{i}=g_{z}^{i}$ and $g_{4}^{i}+i g_{5}^{i}=g_{\bar{z}}^{i}$, and work out the $S O(5)$-gamma matrices as follows,

$$
\left(g_{a}^{i} g_{a}^{i} I+i g_{4}^{i} g_{5}^{j} \Gamma^{i j}\right) \psi_{ \pm}=N_{\mp}^{2} \psi_{ \pm}
$$

In view of the relation,

$$
\left(i g_{4}^{i} g_{5}^{j} \Gamma^{i j}\right)^{2}=\Delta^{2} I \quad \quad \Delta^{2} \equiv g_{4}^{i}\left(g_{4}^{i} g_{5}^{j}-g_{4}^{j} g_{5}^{i}\right) g_{5}^{j}
$$


and hermiticity of $i g_{4}^{i} g_{5}^{j} \Gamma^{i j}$, the real function $\Delta^{2}$ is non-negative. Since $i g_{4}^{i} g_{5}^{j} \Gamma^{i j}$ is traceless, its eigenvalues are $+\Delta$ and $-\Delta$, both with multiplicity 2 . The case $\Delta=0$, for which $g_{4}^{i}$ and $g_{5}^{i}$ are parallel, does not lead to interesting solutions. Thus, we shall assume $\Delta \neq 0$. Choosing a basis of four eigenvectors $\phi_{\sigma}^{( \pm)}$, with $\sigma=1,2$, we obtain,

$$
\left(i g_{4}^{i} g_{5}^{j} \Gamma^{i j}\right) \phi_{\sigma}^{( \pm)}= \pm \Delta \phi_{\sigma}^{( \pm)}
$$

we may decompose $\psi_{ \pm}$as follows,

$$
\psi_{ \pm}=\sum_{\sigma=1,2} \sum_{\eta= \pm} \psi_{ \pm, \sigma}^{(\eta)} \phi_{\sigma}^{(\eta)}
$$

This results in the following conditions on the components,

$$
\left(g_{a}^{i} g_{a}^{i}+\eta \Delta-N_{\mp}^{2}\right) \psi_{ \pm, \sigma}^{(\eta)}=0
$$

Using (4.13), and the definition of $N_{ \pm}^{2}$ in (4.14), we readily derive the relation $\left|g_{z}^{i} g_{z}^{i}\right|^{2}=$ $N_{+}^{2} N_{-}^{2}$, which may also be recast as follows,

$$
\left(g_{a}^{i} g_{a}^{i}+\Delta\right)\left(g_{b}^{j} g_{b}^{j}-\Delta\right)=N_{+}^{2} N_{-}^{2}
$$

We are now in a position to analyze and solve equations (4.20).

Neither $\psi_{+}$nor $\psi_{-}$can vanish identically, since then both would have to vanish by (4.12). Without loss of generality, we may assume that at least some component with $\eta=+$ is non-

vanishing, since this condition may always be achieved possibly upon reversing the sign of $\Delta$, which had not been fixed yet. Thus, we have $g_{a}^{i} g_{a}^{i}+\Delta-N_{-}^{2}=0$. Using the relation (4.21), we find also $g_{a}^{i} g_{a}^{i}-\Delta-N_{+}^{2}=0$, so that $N_{-}^{2}-N_{+}^{2}=2 \Delta \neq 0$. As a result, we have $g_{a}^{i} g_{a}^{i} \pm \Delta-N_{ \pm}^{2} \neq 0$, so that we must have for both $\sigma=1,2$,

$$
\psi_{-, \sigma}^{(+)}=\psi_{+, \sigma}^{(-)}=0
$$

This result is consistent with the fact that $g_{z}^{i} \Gamma_{i}$ and $g_{\bar{z}}^{i} \Gamma_{i}$ anti-commute with $i g_{4}^{i} g_{5}^{j} \Gamma^{i j}$.

\subsection{Solving the remaining differential equations}

The remaining differential equations $\left(a z_{2}\right)$ and $\left(a \bar{z}_{1}\right)$, with $\xi$ eliminated in terms of $\psi$ using (4.2), are given as follows,

$$
\begin{aligned}
\left(a z_{2}\right) \quad 0 & =D_{z} \psi_{+}-\frac{i}{2} \hat{\omega}_{z} \psi_{+}-\frac{1}{4} q_{z}^{i j} \Gamma_{i j} \psi_{+} \\
0 & =D_{z} \psi_{-}-\frac{i}{2} \hat{\omega}_{z} \psi_{-}-\frac{1}{4} q_{z}^{i j} \Gamma_{i j} \psi_{-} \\
\left(a \bar{z}_{1}\right) \quad 0 & =D_{\bar{z}} \psi_{+}+\frac{i}{2} \hat{\omega}_{\bar{z}} \psi_{+}+\left(D_{\bar{z}} \ln Y\right) \psi_{+}-\frac{1}{4} q_{\bar{z}}^{i j} \Gamma_{i j} \psi_{+} \\
0 & =D_{\bar{z}} \psi_{-}+\frac{i}{2} \hat{\omega}_{\bar{z}} \psi_{-}-\left(D_{\bar{z}} \ln Y^{*}\right) \psi_{-}-\frac{1}{4} q_{\bar{z}}^{i j} \Gamma_{i j} \psi_{-}
\end{aligned}
$$


We shall now express these equations in the basis provided by the decomposition of (4.19) with (4.22).

To do so, it will be convenient to make a choice of $S O(5)$ gauge. This is permitted since the consistent formulation of the scalar fields $V$ demands gauge covariance of the BPS equations under local $S O(5)$ gauge transformations. The functions $g_{a}^{i}$ transform homogeneously as a 5 -vector of $S O(5)$. Thus, we may choose a gauge in which only the first component, $i=1$, of $g_{4}^{i}$ is non-vanishing, which leaves invariance under a residual $S O(4)$. We may use this invariance in turn to choose a gauge in which the first two components $i=1,2$ of $g_{5}^{i}$ are non-vanishing. Actually, it will turn out to be slightly more convenient to leave the first two components, $i=1,2$, of $g_{a}^{i}$ non-zero, leaving over a residual $S O(2) \times S O(3)$ gauge freedom. We note that, generally in a theory with holomorphic and harmonic functions, such gauge choices may upset holomorphicity and harmonicity, but here this will not be the case.

The presence of the residual gauge symmetry $S O(2) \times S O(3)$ forces the reduced BPS equations in this gauge to have this symmetry manifest. Thus, in this gauge, the residual BPS equations must be invariant under the $S O(2)$ generator $\Gamma^{12}$. As a result of this invariance, we find that the $S O(5)$-connection is consistently reduced to an $S O(2) \times S O(3)$-connection,

$$
q_{z}^{13}=q_{z}^{14}=q_{z}^{15}=q_{z}^{23}=q_{z}^{24}=q_{z}^{25}=0
$$

Following the conventions of Appendix A, the $S O(2) \times S O(3)$ generators take the form,

$$
\begin{aligned}
\Gamma^{12}=i \sigma_{3} \otimes I & \Gamma^{34}=I \otimes i \sigma_{3} \\
& \Gamma^{45}=I \otimes i \sigma_{1} \\
& \Gamma^{53}=I \otimes i \sigma_{2}
\end{aligned}
$$

With this gauge choice, and the sign choice $\Delta=-g_{4}^{1} g_{5}^{2}+g_{4}^{2} g_{5}^{1}$ in (4.18), we find that the basis of $\phi_{ \pm, \sigma}^{(\eta)}$, namely,

$$
\left(\sigma_{3} \otimes I\right) \phi_{\sigma}^{(\eta)}=\eta \phi_{\sigma}^{(\eta)}
$$

so that we have $\psi_{+, \alpha}^{(-)}=\psi_{-, \alpha}^{(+)}=0$. In this basis, the remaining equations in (4.23) decompose as follows,

$$
\begin{aligned}
\left(a z_{2}\right) \quad 0 & =\left(D_{z}-\frac{i}{2} \hat{\omega}_{z}-\frac{i}{2} q_{z}^{12}-\mathcal{A}_{z}\right) \psi_{+}^{(+)} \\
0 & =\left(D_{z}-\frac{i}{2} \hat{\omega}_{z}+\frac{i}{2} q_{z}^{12}-\mathcal{A}_{z}\right) \psi_{-}^{(-)} \\
\left(a \bar{z}_{1}\right) \quad 0 & =\left(D_{\bar{z}}+\frac{i}{2} \hat{\omega}_{\bar{z}}-\frac{i}{2} q_{\bar{z}}^{12}-\mathcal{A}_{\bar{z}}+D_{\bar{z}} \ln Y\right) \psi_{+}^{(+)} \\
0 & =\left(D_{\bar{z}}+\frac{i}{2} \hat{\omega}_{\bar{z}}+\frac{i}{2} q_{\bar{z}}^{12}-\mathcal{A}_{\bar{z}}-D_{\bar{z}} \ln Y^{*}\right) \psi_{-}^{(-)}
\end{aligned}
$$


where we have denoted the remaining $S O(3)$-connection by

$$
\mathcal{A}_{a}=\frac{i}{2}\left(q_{a}^{34} \sigma^{3}+q_{a}^{45} \sigma^{1}+q_{a}^{53} \sigma^{2}\right)
$$

From equation (4.12), we have a relation between $\psi_{+}^{(+)}$and $\psi_{-}^{(-)}$given by,

$$
\psi_{-}^{(-)}=X \psi_{+}^{(+)} \quad X \equiv-i\left(g_{z}^{1}+i g_{z}^{2}\right) \frac{f_{1}-f_{2}}{D_{z}\left(f_{1}+f_{2}\right)}
$$

Eliminating $\psi_{-}^{(-)}$in equations (4.27) in favor of $\psi_{+}^{(+)}$and $X$, and taking the sum or difference between similar equations, we find the differential equations,

$$
\begin{aligned}
0 & =\left(D_{z}-\frac{i}{2} \hat{\omega}_{z}-\frac{i}{2} q_{z}^{12}-\mathcal{A}_{z}\right) \psi_{+}^{(+)} \\
0 & =\left(D_{\bar{z}}+\frac{i}{2} \hat{\omega}_{\bar{z}}-\frac{i}{2} q_{\bar{z}}^{12}-\mathcal{A}_{\bar{z}}+D_{\bar{z}} \ln Y\right) \psi_{+}^{(+)}
\end{aligned}
$$

as well as the equations,

$$
\begin{aligned}
& q_{z}^{12}=+i D_{z} \ln X \\
& q_{\bar{z}}^{12}=-i D_{\bar{z}} \ln \bar{X}
\end{aligned}
$$

In order to solve these equations, it is useful to rewrite the $D_{\bar{z}} Y$ term in equation (4.30) using the relation (4.5). We obtain

$$
D_{\bar{z}} \ln Y=\frac{1}{2} D_{\bar{z}} \ln \frac{f_{1}-f_{2}}{f_{1}+f_{2}}+\frac{1}{2} D_{\bar{z}} \ln \frac{c(w)}{\bar{c}(\bar{w})}
$$

At this point, it is helpful to introduce the notation

$$
\Psi_{+}^{(+)}=\left\{\frac{i \rho X}{c(\bar{w})}\right\}^{\frac{1}{2}} \psi_{+}^{(+)}
$$

Equations (4.30) now assume a particularly simple form,

$$
\begin{aligned}
& \left(\partial_{w}-\mathcal{A}_{w}\right) \Psi_{+}^{(+)}=0 \\
& \left(\partial_{\bar{w}}-\mathcal{A}_{\bar{w}}\right) \Psi_{+}^{(+)}=0
\end{aligned}
$$

where we have set $\mathcal{A}_{w}=\rho \mathcal{A}_{z}$ and $\mathcal{A}_{\bar{w}}=\rho \mathcal{A}_{\bar{z}}$. These equations pose no further restriction on the number of independent components of $\Psi_{+}^{(+)}$provided that the integrability condition

$$
\partial_{w} \mathcal{A}_{\bar{w}}-\partial_{\bar{w}} \mathcal{A}_{w}-\mathcal{A}_{w} \mathcal{A}_{\bar{w}}+\mathcal{A}_{\bar{w}} \mathcal{A}_{w}=0
$$

is obeyed. Hence, $\mathcal{A}$ needs to be pure gauge and there must exist an $S O(3)$ gauge transformation such that $\mathcal{A}=0$ or, equivalently,

$$
q_{z}^{34}=q_{z}^{45}=q_{z}^{53}=0
$$

In other words, barring global issues, it is possible to find a gauge in which all the components of $q_{z}^{i j}$ are identically zero, with the exception of $q_{z}^{12}$. In this gauge, $\Psi_{+}^{(+)}$is a constant spinor. 


\subsection{Solving for $p_{a}^{i r}$ and $\tilde{g}_{a}^{r}$}

The equations $\left(c_{1}\right)$ and $\left(c_{2}\right)$ in (3.25) can be simplified by eliminating $\xi$ in favor of $\psi$ of $Y$, using equations (4.3), and we find,

$$
\begin{aligned}
\left(c_{1}\right) \quad 0 & =\frac{1}{\sqrt{2}} p_{z}^{i r} \Gamma_{i} \psi_{+}+i \tilde{g}_{z}^{r} \psi_{-} \\
0 & =\frac{1}{\sqrt{2}} p_{z}^{i r} \Gamma_{i} \psi_{-}-i \tilde{g}_{z}^{r} \psi_{+} \\
\left(c_{2}\right) \quad 0 & =\frac{|Y|^{2}}{\sqrt{2}} p_{\bar{z}}^{i r} \Gamma_{i} \psi_{+}+i \tilde{g}_{\bar{z}}^{r} \psi_{-} \\
0 & =\frac{1}{\sqrt{2}} p_{\bar{z}}^{i r} \Gamma_{i} \psi_{-}-i|Y|^{2} \tilde{g}_{\bar{z}}^{r} \psi_{+}
\end{aligned}
$$

Next, we use the decomposition of (4.19) with the projector conditions of (4.22), and the explicit characterization (4.26) of the basis spinors $\phi_{\sigma}^{( \pm)}$, to analyze (4.37). Clearly, the equations decompose onto $\phi_{\sigma}^{( \pm)}$as follows,

$$
\begin{array}{ll}
\phi_{\sigma}^{(+)} & \psi_{+}, \Gamma^{3} \psi_{+}, \Gamma^{4} \psi_{+}, \Gamma^{5} \psi_{+}, \Gamma^{1} \psi_{-}, \Gamma^{2} \psi_{-} \\
\phi_{\sigma}^{(-)} & \psi_{-}, \Gamma^{3} \psi_{-}, \Gamma^{4} \psi_{-}, \Gamma^{5} \psi_{-}, \Gamma^{1} \psi_{+}, \Gamma^{2} \psi_{+}
\end{array}
$$

As a result, we immediately have,

$$
\begin{aligned}
& 0=\left(p_{z}^{3 r} \sigma_{1}+p_{z}^{4 r} \sigma_{2}+p_{z}^{5 r} \sigma_{3}\right) \psi_{+}^{(+)} \\
& 0=\left(p_{\bar{z}}^{3 r} \sigma_{1}+p_{\bar{z}}^{4 r} \sigma_{2}+p_{\bar{z}}^{5 r} \sigma_{3}\right) \psi_{+}^{(+)}
\end{aligned}
$$

Since $\psi_{+}^{(+)} \neq 0$, it follows that

$$
p_{z}^{3 r}=p_{z}^{4 r}=p_{z}^{5 r}=0
$$

In the remaining equations, we eliminate $\psi_{-}$in favor of $\psi_{+}$and $X$, using (4.29), resulting in a set of four equations which are all proportional to $\psi_{+}^{(+)}$. Again, since this quantity is non-vanishing, it may be omitted, and we obtain the following equations on the coefficients,

$$
\begin{aligned}
\left(c_{1}\right) \quad 0 & =\frac{1}{\sqrt{2}}\left(p_{z}^{1 r}+i p_{z}^{2 r}\right)+i \tilde{g}_{z}^{r} X \\
0 & =\frac{1}{\sqrt{2}}\left(p_{z}^{1 r}-i p_{z}^{2 r}\right) X-i \tilde{g}_{z}^{r} \\
\left(c_{2}\right) \quad 0 & =\frac{|Y|^{2}}{\sqrt{2}}\left(p_{\bar{z}}^{1 r}+i p_{\bar{z}}^{2 r}\right)+i \tilde{g}_{\bar{z}}^{r} X \\
0 & =\frac{1}{\sqrt{2}}\left(p_{\bar{z}}^{1 r}-i p_{\bar{z}}^{2 r}\right) X-i|Y|^{2} \tilde{g}_{\bar{z}}^{r}
\end{aligned}
$$


Compatibility of the first and the fourth equations of (4.41) requires

$$
|X|^{2}=|Y|^{2}
$$

which in turn implies the consistency of the second and third equations. The remaining equations give $p_{z}^{1 r}$ and $p_{z}^{2 r}$ in terms of $\tilde{g}_{z}^{r}$, and may be summarized as follows,

$$
\frac{1}{\sqrt{2}}\left(p_{z}^{1 r}+i \alpha p_{z}^{2 r}\right)=-i \alpha X^{\alpha} \tilde{g}_{z}^{r} \quad \alpha=+,-
$$

which concludes the complete parametrization of the solutions to the BPS equations, in terms of a single complex function $X$. A summary of the solution will be the starting point of the subsequent section. 


\section{Constructing the local solution}

We begin by summarizing the complete solution to the BPS equations, obtained in the previous section. Combining these results with the Bianchi identities, and the general structure of the scalar field equations, we produce the complete solution to all the BPS, Bianchi and field equations. We will work for a general number of tensor multiplets $m$.

\subsection{Summary of the solutions to the BPS equations}

Given the solutions to the BPS equations with the gauge choice $g_{a}^{i}=0$ for $i=3,4,5$, and the resulting solutions $p_{a}^{i r}=0$ for $i=3,4,5$, the basis of frames takes the simplified form,

$$
D_{a} V V^{-1}=\left(\begin{array}{ccc}
q_{a}^{i j} & 0 & \sqrt{2} p_{a}^{i s} \\
0 & 0 & 0 \\
\sqrt{2} p_{a}^{j r} & 0 & s_{a}^{r s}
\end{array}\right)
$$

where $i, j=1,2$ and $r, s=6, \cdots, m+5$. The block $q_{a}^{i j}$ generates an $S O(2)$-connection, namely $S O(2)_{q}$, which we shall abbreviate as follows,

$$
\begin{aligned}
& q_{z}=q_{z}^{12}=+i D_{z} \ln X \\
& q_{\bar{z}}=q_{\bar{z}}^{12}=-i D_{\bar{z}} \ln \bar{X}
\end{aligned}
$$

As a result, the frame $V$ itself takes the reduced form,

$$
V=\left(\begin{array}{ccc}
V^{i}{ }_{I} & 0 & V^{i}{ }_{R} \\
0 & I_{3} & 0 \\
V^{r}{ }_{I} & 0 & V^{r}{ }_{R}
\end{array}\right)
$$

where $i, I=1,2$, and $r, R=6, \cdots, m+5$. The block $I_{3}$ represents the identity in the indices $i, I=3,4,5$. The defining property for $S O(5, m)$ is $V^{-1}=\eta V^{t} \eta$ where $\eta=\operatorname{diag}\left(I_{5},-I_{m}\right)$. It will be useful to write in components both equations $V V^{-1}=V^{-1} V=I$,

$$
\begin{aligned}
& V^{i}{ }_{I} V^{j}{ }_{I}-V_{R}^{i} V_{R}^{j}=\delta^{i j} \quad V^{i}{ }_{I} V^{i}{ }_{J}-V^{r}{ }_{I} V^{r}{ }_{J}=\delta_{I J} \\
& V_{R}^{i} V_{R}^{r}-V_{I}^{i} V_{I}^{r}=0 \quad V^{i}{ }_{I} V^{i}{ }_{R}-V^{r}{ }_{I} V^{r}{ }_{R}=0 \\
& V^{r}{ }_{R} V^{s}{ }_{R}-V^{r}{ }_{I} V^{s}{ }_{R}=\delta^{r s} \quad V^{r}{ }_{R} V^{r}{ }_{S}-V_{R}^{i} V_{S}^{i}=\delta_{R S}
\end{aligned}
$$

Summation over repeated indices is assumed here; specifically over the indices $I=1,2$ and $R=6, \cdots, m+5$ in the left column, and over the indices $i=1,2$ and $r=6, \cdots, m+5$ in the right column. Using the same conventions, the expressions for $q, p, s$ are found to be,

$$
\begin{aligned}
\sqrt{2} p_{a}^{i r} & =\left(D_{a} V^{i}{ }_{R}\right) V^{r}{ }_{R}-\left(D_{a} V^{i}{ }_{I}\right) V^{r}{ }_{I} \\
q_{a}^{i j} & =\left(D_{a} V_{I}^{i}\right) V^{j}{ }_{I}-\left(D_{a} V^{i}{ }_{R}\right) V^{j}{ }_{R} \\
s_{a}^{r s} & =\left(D_{a} V^{r}{ }_{R}\right) V^{s}{ }_{R}-\left(D_{a} V^{r}{ }_{I}\right) V^{s}{ }_{I}
\end{aligned}
$$


It will be natural to decompose all fields in a basis in which the $S O(2)_{q}$ action is diagonal. To this end, we introduce with $\alpha=+,-$,

$$
\begin{aligned}
g_{z}^{\alpha} & =\frac{1}{\sqrt{2}}\left(g_{z}^{1}+i \alpha g_{z}^{2}\right) & g_{\bar{z}}^{\alpha} & =\frac{1}{\sqrt{2}}\left(g_{\bar{z}}^{1}+i \alpha g_{\bar{z}}^{2}\right) \\
p_{z}^{\alpha r} & =\frac{1}{\sqrt{2}}\left(p_{z}^{1 r}+i \alpha p_{z}^{2 r}\right) & p_{\bar{z}}^{\alpha r} & =\frac{1}{\sqrt{2}}\left(p_{\bar{z}}^{1 r}+i \alpha p_{\bar{z}}^{2 r}\right)
\end{aligned}
$$

As a result of the BPS equations, the fluxes $g_{a}^{\alpha}$ may then be parametrized by the field $X$, and the radii $f_{1}$ and $f_{2}$, as follows,

$$
\begin{aligned}
g_{z}^{+} & =i \frac{X}{\sqrt{2}} \frac{D_{z}\left(f_{1}+f_{2}\right)}{f_{1}-f_{2}} & g_{z}^{-} & =-i \frac{\bar{X}}{\sqrt{2}} \frac{D_{z}\left(f_{1}-f_{2}\right)}{f_{1}-f_{2}} \\
g_{\bar{z}}^{+} & =i \frac{X}{\sqrt{2}} \frac{D_{\bar{z}}\left(f_{1}-f_{2}\right)}{f_{1}-f_{2}} & g_{\bar{z}}^{-} & =-i \frac{\bar{X}}{\sqrt{2}} \frac{D_{\bar{z}}\left(f_{1}+f_{2}\right)}{f_{1}-f_{2}}
\end{aligned}
$$

As a result of $(4.5),(4.6)$, and (4.42), the radii $f_{1}, f_{2}$, the metric $\rho^{2}$, and the field $X$ are related as follows,

$$
f_{1} f_{2}=H \quad|X|^{2}=\frac{f_{1}-f_{2}}{f_{1}+f_{2}} \quad \rho^{2}\left(f_{1}^{2}-f_{2}^{2}\right)=\left|\partial_{w} H\right|^{2}
$$

By the solution to the BPS equations of (4.43), the functions $p_{a}^{i r}$ and $\tilde{g}_{a}^{r}$ are related as follows,

$$
\begin{array}{lll}
p_{z}^{\alpha r}=-i \alpha X^{\alpha} \tilde{g}_{z}^{r} & \\
p_{\bar{z}}^{\alpha r}=-i \alpha \bar{X}^{-\alpha} \tilde{g}_{\bar{z}}^{r} & \alpha=+,-
\end{array}
$$

\subsection{Solving for $V$ and $|X|$}

We begin by eliminating $\tilde{g}_{a}^{r}$ between $p_{a}^{ \pm r}$, and find,

$$
p_{z}^{+r}=-X^{2} p_{z}^{-r}
$$

together with its complex conjugate. Next, we write out this equation in terms of the frame variables, and we find,

$$
-\left(D_{z} V^{+}{ }_{I}\right) V_{I}^{r}+\left(D_{z} V^{+}{ }_{R}\right) V_{R}^{r}=X^{2}\left(\left(D_{z} V^{-}{ }_{I}\right) V_{I}^{r}-\left(D_{z} V^{-}{ }_{R}\right) V^{r}{ }_{R}\right)
$$

\subsubsection{Solving for $V^{i}{ }_{I}$}

Contracting (5.12) by $V_{J}^{r}$, and using the top two relations on the right of (5.4), we find,

$$
\begin{aligned}
D_{z} V^{+}{ }_{I} & -\left[\left(D_{z} V^{+}{ }_{J}\right) V^{-}{ }_{J}-\left(D_{z} V^{+}{ }_{R}\right) V^{-}{ }_{R}\right] V^{+}{ }_{I} \\
& =-X^{2}\left(D_{z} V^{-}{ }_{I}-\left[\left(D_{z} V^{-}{ }_{J}\right) V^{+}{ }_{J}-\left(D_{z} V^{-}{ }_{R}\right) V^{+}{ }_{R}\right] V^{-}{ }_{I}\right)
\end{aligned}
$$


Recognizing the following combinations,

$$
\begin{aligned}
& q_{z}^{+-}=\left(D_{z} V^{+}{ }_{J}\right) V^{-}{ }_{J}-\left(D_{z} V^{+}{ }_{R}\right) V^{-}{ }_{R} \\
& q_{z}^{-+}=\left(D_{z} V^{-}{ }_{J}\right) V^{+}{ }_{J}-\left(D_{z} V^{-}{ }_{R}\right) V^{+}{ }_{R} \\
& q_{z}^{+-}=-q_{z}^{-+}=-i q_{z}^{12}=D_{z} \ln X
\end{aligned}
$$

equation (5.13) takes the following form,

$$
D_{z} V^{+}{ }_{I}-\left(D_{z} \ln X\right) V^{+}{ }_{I}=-X^{2}\left(D_{z} V^{-}{ }_{I}+\left(D_{z} \ln X\right) V^{-}{ }_{I}\right)
$$

which may also be rewritten (in components) as,

$$
D_{z}\left(\frac{V^{+} I}{X}+X V^{-}{ }_{I}\right)=0
$$

These are Cauchy-Riemann equations, and are solved by a pair of arbitrary holomorphic functions $\lambda_{I}$, with $I=1,2$. In local complex coordinates $w, \bar{w}$ on $\Sigma$, the Cauchy-Riemann equations simply read $\partial_{\bar{w}} \lambda_{I}=0$. As a result, we have,

$$
\begin{aligned}
& \frac{V^{+}{ }_{I}}{X}+X V^{-}{ }_{I}=\bar{\lambda}_{I} \\
& \frac{V^{-}{ }_{I}}{\bar{X}}+\bar{X} V^{+}{ }_{I}=\lambda_{I}
\end{aligned}
$$

They are solved by,

$$
\begin{aligned}
& \left(1-|X|^{4}\right) V^{+}{ }_{I}=X \bar{\lambda}_{I}-X|X|^{2} \lambda_{I} \\
& \left(1-|X|^{4}\right) V_{I}^{-}=\bar{X} \lambda_{I}-\bar{X}|X|^{2} \bar{\lambda}_{I}
\end{aligned}
$$

\subsubsection{Solving for $V_{R}^{i}$}

Next, we contract (5.12) by $V^{r}$, use the bottom two relations in the right column of (5.4), and employ the combinations of (5.13) to recast the equations in the following form,

$$
D_{z}\left(\frac{V^{+} R}{X}+X V^{-}{ }_{R}\right)=0
$$

which are solved by further arbitrary holomorphic functions $\lambda_{R}$, with $R=6, \cdots, m+5$,

$$
\frac{V^{+} R}{X}+X V^{-}{ }_{R}=\bar{\lambda}_{R}
$$

giving rise to the following explicit solutions,

$$
\begin{aligned}
& \left(1-|X|^{4}\right) V^{+}{ }_{R}=X \bar{\lambda}_{R}-X|X|^{2} \lambda_{R} \\
& \left(1-|X|^{4}\right) V_{R}^{-}=\bar{X} \lambda_{R}-\bar{X}|X|^{2} \bar{\lambda}_{R}
\end{aligned}
$$




\subsubsection{Solving for $|X|$}

Having now obtained explicit solutions for the fields $V^{ \pm}{ }_{I}$ and $V^{ \pm}{ }_{R}$ in terms of holomorphic functions $\lambda_{I}, \lambda_{R}$, their complex conjugates, and the function $X$, we may use the top left relation of (5.4) to determine $|X|$. In \pm components, this equation amounts to one complex and one real equation, respectively given by,

$$
\begin{aligned}
& V^{+}{ }_{I} V^{+}{ }_{I}-V^{+}{ }_{R} V^{+}{ }_{R}=0 \\
& V^{+}{ }_{I} V^{-}{ }_{I}-V^{+}{ }_{R} V^{-}{ }_{R}=1
\end{aligned}
$$

Using the explicit solutions of (5.18) and (5.21), the first equation becomes,

$$
\bar{\lambda} \cdot \bar{\lambda}+|X|^{4} \lambda \cdot \lambda-2|X|^{2} \lambda \cdot \bar{\lambda}=0
$$

where we have omitted overall factors of powers of $\left(1-|X|^{4}\right)$, since they are non-vanishing, and the functions $\lambda^{A}, \bar{\lambda}^{A}$ are contracted with the $S O(2, m)$-invariant metric $\eta$. Vanishing of the imaginary part of (5.23) implies, $\operatorname{Im}(\lambda \cdot \lambda)=0$. Since $\lambda \cdot \lambda$ is holomorphic, this can only be realized by having this quantity be a real constant $b$, so that $\lambda \cdot \lambda=b$. The real part of (5.23) and the second equation of (5.22) then become respectively,

$$
\begin{aligned}
b\left(1+|X|^{4}\right)-2|X|^{2} \lambda \cdot \bar{\lambda} & =0 \\
\left(1+|X|^{4}\right)|X|^{2} \lambda \cdot \bar{\lambda}-2 b|X|^{4} & =\left(1-|X|^{4}\right)^{2}
\end{aligned}
$$

Eliminating $\lambda \cdot \bar{\lambda}$ between the equations of (5.24) gives a relation involving only $|X|$, which can hold for non-trivial $|X|$ if and only if $b=2$. As a result, $|X|$ is determined in terms of $m+2$ holomorphic functions $\lambda_{A}$ as follows,

$$
\begin{aligned}
& \lambda \cdot \lambda=2 \\
& \lambda \cdot \bar{\lambda}=|X|^{2}+|X|^{-2}
\end{aligned}
$$

By construction of the local solution, $|X|$ is real and positive and hence $\lambda \cdot \bar{\lambda} \geq 2$.

\subsubsection{Solving for $V^{r}$ and $V_{R}^{r}$}

The relations (5.4) between the scalar fields allow us to solve for $V^{r}{ }_{A}$ in terms of $V^{i}{ }_{A}$ via smooth relations. By first diagonalizing the real symmetric matrix $V^{r}{ }_{R} V_{S}^{r}=\delta_{R S}+V_{R}^{i} V^{i}{ }_{S}$ by an orthogonal matrix $M_{1}$ and a real diagonal matrix $D$, we find

$$
\begin{aligned}
V_{R}^{r} & =\left(M_{2}\right)^{r}{ }_{s} D^{s}{ }_{S}\left(M_{1}\right)_{R}{ }_{R} \\
V^{r}{ }_{I} & =\left(M_{2}\right)^{r}{ }_{s}\left(D^{-1}\right)^{s}{ }_{R}\left(M_{1}\right)^{R}{ }_{S} V^{i}{ }_{T} V^{j}{ }_{I} \delta^{S T} \delta_{i j}
\end{aligned}
$$

where $M_{2}$ is a second orthogonal matrix, and where summation is implied for any pair of repeated raised and lowered indices. 


\subsection{Calculating the flux potentials}

Conserved charges arise from integrating the closed three-form fields $G^{A}$ of (2.8) over closed compact three-cycles, such as the asymptotic $S^{3}$ components of the boundary. The field $G^{A}$ may be obtained by inverting relations of (2.9), and expressing $H^{i}$ and $H^{r}$ in terms of (3.4),

$$
\eta_{A B} G^{B}=+\left(V_{A}^{i} g_{a}^{i}-V^{r}{ }_{A} \tilde{g}_{a}^{r}\right) f_{1}^{2} \hat{e}^{01} e^{a}+\left(V^{i}{ }_{A} h_{a}^{i}-V^{r}{ }_{A} \tilde{h}_{a}^{r}\right) f_{2}^{2} \hat{e}^{23} e^{a}
$$

The forms $\hat{e}^{01}$ and $\hat{e}^{23}$ are the unit volume forms respectively on $A d S_{2}$ and $S^{2}$. They are real and closed by construction, so that $G^{A}$ may be recast in terms of derivatives of real-valued flux potentials $\Phi^{A}$ and $\Psi^{A}$, which are functions of $\Sigma$ only,

$$
G^{A}=d \Psi^{A} \wedge \hat{e}^{01}+d \Phi^{A} \wedge \hat{e}^{23}
$$

The conserved charges of interest here arise from the contributions above which are proportional to the volume form $\hat{e}^{23}$ on $S^{2}$. Since the volume form $\hat{e}^{01}$ assigns infinite volume to $A d S_{2}$, its contributions does not lead to finite conserved charges. Thus, we focus on the behavior of $\Phi^{A}$, which may be determined from the differential equations,

$$
\eta_{A B} D_{z} \Phi^{B}=-i f_{2}^{2}\left(V_{A}^{i} g_{z}^{i}+V_{A}^{r} \tilde{g}_{z}^{r}\right)
$$

where we have used (3.5) to re-express $h_{a}^{i}$ and $\tilde{h}_{a}^{r}$ in terms of $g_{a}^{i}$ and $\tilde{g}_{a}^{r}$ respectively.

We now seek to solve for $\Phi^{A}$ in terms of the harmonic function $H$, the holomorphic functions $\lambda_{A}$ and their complex conjugates. To do so, we express the components of $g_{z}^{i}$ given in (5.8) in terms of $H$ and $|X|$ using the following formulas,

$$
\begin{aligned}
& \left(f_{1}+f_{2}\right)^{2}=\frac{4 H}{1-|X|^{4}} \\
& \left(f_{1}-f_{2}\right)^{2}=\frac{4 H|X|^{4}}{1-|X|^{4}}
\end{aligned}
$$

As a result, we have

$$
\begin{aligned}
& g_{z}^{+}=+\frac{i X}{2 \sqrt{2}|X|^{2}} D_{z} \ln \left(\frac{4 H}{1-|X|^{4}}\right) \\
& g_{z}^{-}=-\frac{i \bar{X}}{2 \sqrt{2}} D_{z} \ln \left(\frac{4 H|X|^{4}}{1-|X|^{4}}\right)
\end{aligned}
$$

To compute the flux potentials, only the general relations obeyed by $V^{r} A$ will be needed, but not their explicit forms of (5.26). We compute $V^{r}{ }_{A} \tilde{g}_{z}^{r}$ using (5.10) and (5.5),

$$
V_{A}^{r} \tilde{g}_{z}^{r}=\frac{i}{X} V_{A}^{r} p_{z}^{+r}=\frac{i}{\sqrt{2} X}\left(-D_{z} V^{+}{ }_{J} V^{r}{ }_{A} V^{r}{ }_{J}+D_{z} V^{+}{ }_{R} V_{A}^{r} V_{R}^{r}\right)
$$


We now make use of the $S O(2, m)$ relations in the right column of (5.4). Recognizing again the combinations of (5.14), we obtain,

$$
V_{A}^{r} \tilde{g}_{z}^{r}=\frac{i}{\sqrt{2}} D_{z}\left(\frac{V^{+} A}{X}\right)
$$

Using (5.29), (5.31), (5.33), and the expressions for $V_{I}^{i}$ and $V_{R}^{i},(5.18)$ and (5.21), we obtain a formula for the derivative of the potential $\Phi^{A}=\eta^{A B} \Phi_{B}$ in terms of $\lambda_{A}, H$ and $|X|$,

$$
D_{z} \Phi_{A}=D_{z}\left(-\frac{1}{\sqrt{2}} \frac{H|X|^{2}\left(\lambda_{A}+\bar{\lambda}_{A}\right)}{\left(1+|X|^{2}\right)^{2}}\right)+\frac{1}{2 \sqrt{2}} \lambda_{A} D_{z} H
$$

Next, we use the second formula of (5.25) to express $|X|$ in terms of $\lambda \cdot \bar{\lambda}$, and to obtain an expression for $D_{z} \Phi^{A}$ in terms of only $\lambda_{A}, \bar{\lambda}_{A}$ and $H$. Integrating this equation, we find the following final form for the flux potentials,

$$
\Phi_{A}=\tilde{\Phi}_{A}-\sqrt{2} \frac{H \operatorname{Re}\left(\lambda_{A}\right)}{\lambda \cdot \bar{\lambda}+2} \quad \tilde{\Phi}_{A}=\frac{1}{2 \sqrt{2}} \int \lambda_{A} \partial_{w} H+\text { c.c. }
$$

Note that the contributions $\tilde{\Phi}_{A}$ are real harmonic functions on $\Sigma$. An integration constant may be added to each $\tilde{\Phi}_{A}$, which amounts to a gauge transformation on the two-form field $B^{A}$, and does not affect the field strengths $G^{A}$, or $H^{i}, H^{r}$.

\subsection{Summary of the local solution}

The data of the general local solution are the real harmonic function $H$, and an $S O(2, m)$ vector of (locally) holomorphic functions $\lambda_{A}$ which must satisfy the following conditions,

$$
\lambda \cdot \lambda=2 \quad \lambda \cdot \bar{\lambda} \geq 2
$$

Combining the formulas for the radii $f_{1}, f_{2}$ and worldsheet metric $\rho^{2}$ of $(5.9)$ with the expression for $|X|$ in terms the harmonic function $H$ and the holomorphic functions $\lambda_{A}$, we find the following explicit expressions for the radii and metric,

$$
\begin{aligned}
f_{1}^{4} & =H^{2} \frac{\lambda \cdot \bar{\lambda}+2}{\lambda \cdot \bar{\lambda}-2} \\
f_{2}^{4} & =H^{2} \frac{\lambda \cdot \bar{\lambda}-2}{\lambda \cdot \bar{\lambda}+2} \\
\rho^{4} & =\frac{\left|\partial_{w} H\right|^{4}}{16 H^{2}}(\lambda \cdot \bar{\lambda}-2)(\lambda \cdot \bar{\lambda}+2)
\end{aligned}
$$

Note that the quantity $\lambda \cdot \bar{\lambda}$ has indefinite metric. 
The solution for the scalar fields may be derived from eliminating $|X|$ in favor of $\lambda \cdot \bar{\lambda}=$ $|X|^{2}+|X|^{-2}$ from (5.18) and (5.21). Overall phases remain in view of the $S O(2)$ gauge non-invariance of the scalar fields. Thus we have,

$$
V^{+}{ }_{A}=X\left(\bar{\lambda}_{A}-|X|^{2} \lambda_{A}\right) /\left(1-|X|^{4}\right)
$$

together with its complex conjugate. Expressions for $V^{r}{ }_{A}$ may be derived using (5.26).

The solutions for the three-form field $G^{A}=d B^{A}$ may be expressed in terms of real-valued flux potential functions $\Phi^{A}$ and $\Psi^{A}$ by (5.28), via the relations,

$$
B^{A}=\Psi^{A} \hat{e}^{01}+\Phi^{A} \hat{e}^{23}
$$

where the flux potential functions for the solution are given by,

$$
\begin{aligned}
\Phi^{A} & =-\sqrt{2} \frac{H \operatorname{Re}\left(\lambda^{A}\right)}{\lambda \cdot \bar{\lambda}+2}+\tilde{\Phi}^{A} & \tilde{\Phi}_{A} & =\frac{1}{2 \sqrt{2}} \int \lambda_{A} \partial_{w} H+\text { c.c. } \\
\Psi^{A} & =-\sqrt{2} \frac{H \operatorname{Im}\left(\lambda^{A}\right)}{\lambda \cdot \bar{\lambda}-2}+\tilde{\Psi}^{A} & \tilde{\Psi}_{A} & =\frac{i}{2 \sqrt{2}} \int \lambda_{A} \partial_{w} H+\text { c.c. }
\end{aligned}
$$

If a closed homology three-cycle $\mathcal{S}$ in the solution manifold (typically a sphere $S^{3}$ ) is parametrized as a two-sphere $S^{2}$ warped over an interval $\mathcal{I}$ in $\Sigma$, then the conserved Page charge (see for example [46]) vector $Q_{\mathcal{S}}^{A}$ through the cycle $\mathcal{S}$ is given by,

$$
Q_{\mathcal{S}}^{A}=\oint_{\mathcal{S}} G^{A}=\sqrt{2} \pi \int_{\mathcal{I}} \lambda^{A} \partial_{w} H+\text { c.c. }
$$

where we have used $\oint_{S^{2}} \hat{e}^{23}=4 \pi$. Generally, the interval $\mathcal{I}$ need not be closed on $\Sigma$.

Finally, we have verified that the local solution obtained here solves the Bianchi identities which have not been solved explicitly in this section, as well as the field equations. The detailed calculations may be found in [47]. 


\section{Topology and regularity assumptions}

In the preceding section, we have obtained the complete and most general local half-BPS solution to six-dimensional $(0,4)$ supergravity with $S O(2,1) \times S O(3)$ symmetry. The solutions are parametrized by a real harmonic function $H$, and $m+2$ holomorphic functions $\lambda_{I}, \lambda_{R}$ on a Riemann surface $\Sigma$ with boundary, subject to the conditions,

$$
\begin{array}{lll}
\lambda \cdot \lambda=\delta_{I J} \lambda^{I} \lambda^{J}-\delta_{R S} \lambda^{R} \lambda^{S}=2 & I, J=1,2 \\
\lambda \cdot \bar{\lambda}=\delta_{I J} \lambda^{I} \bar{\lambda}^{J}-\delta_{R S} \lambda^{R} \bar{\lambda}^{S} \geq 2 & R, S=6, \cdots, m+5
\end{array}
$$

The expressions for the metric factors $f_{1}, f_{2}, \rho$, the scalars, and for the flux potentials $\Phi^{A}, \Psi^{A}$ were given in terms of these functions in (5.37), (5.39), and (5.40). In this section, we spell out the topology and regularity conditions which we shall impose to obtain regular global solutions with prescribed asymptotic behavior.

Motivated by holographic applications in the study of junctions of two-dimensional CFTs, we restrict the topology of solutions to having $N$ distinct asymptotic regions each of which is homeomorphic to $A d S_{3} \times S^{3}$ (namely a maximally symmetric asymptotic solution supported by non-vanishing three-form flux). This condition is the direct six-dimensional analogue of the restriction to locally asymptotically $A d S_{5} \times S^{5}$ for ten-dimensional Type IIB. It is further motivated by the study in $[31,32,37]$ of Type IIB on locally asymptotically $A d S_{3} \times S^{3} \times K 3$.

However, we know from [32] that there exist regular solutions with different asymptotics. At the same time, it may also be possible to find a physical interpretation for some of the singular solutions. The study of solutions with different topologies and asymptotic regions remains an open and interesting problem, which we defer to future work.

\subsection{Basic regularity assumptions}

Motivated by the locally asymptotically $A d S_{3} \times S^{3}$ structure of the solutions, we impose the following basic regularity assumptions on the physical fields needed to achieve regularity under the assumption of this topological structure.

(1) The physical data of the solution, namely the metric factors $f_{1}, f_{2}, \rho$, the scalars $V_{R}^{i}$, and the reduced field strengths $d \Phi^{A}, d \Psi^{A}$ are regular and single-valued inside $\Sigma$.

(2) These physical data extend smoothly to the boundary $\partial \Sigma$, except at isolated points where they may diverge to produce a locally asymptotically $A d S_{3} \times S^{3}$ region.

(3) The condition $f_{1}>0$ holds throughout $\Sigma$ and on the boundary $\partial \Sigma$. 
(4) The condition $f_{2}>0$ holds throughout the interior of $\Sigma$.

The condition $f_{2}=0$ defines the position of the boundary $\partial \Sigma$.

For simplicity, we shall assume here that the boundary $\partial \Sigma$ consists of a single connected component, though we know that this restriction may easily be lifted, as was done in [32].

\subsection{Implications on the (locally) holomorphic data}

The above assumptions on topology and regularity on the physical fields have simple implications on the (locally) holomorphic and harmonic data in terms of which the complete local solutions of the preceding section have been expressed. We now spell out these implications.

(a) The real harmonic function $H$ is smooth, single-valued, and satisfies $H>0$ inside $\Sigma$.

(b) The condition $H=0$ gives an equivalent definition of the position of the boundary $\partial \Sigma$.

(c) The condition $\lambda \cdot \bar{\lambda}>2$ holds inside $\Sigma$.

(d) The relation $\operatorname{Im}\left(\lambda_{A}\right)=0$ holds on $\partial \Sigma$, except at isolated points.

(e) The one-forms $\Lambda_{A} \equiv \lambda_{A} \partial H$ are holomorphic and single-valued inside $\Sigma$. They extend smoothly to $\partial \Sigma$, except at isolated points.

Points (a) and (b) follow from the relation $f_{1} f_{2}=H$ and points (1), (2), (3) and (4) of Section 6.1, while point (c) results upon the use of equation (5.37). Point (d) follows from the regularity of $f_{1}$, which via the first relation of (5.37) requires that $\lambda \cdot \bar{\lambda}-2$ must vanish at the same rate as $H^{2}$ does at the boundary. Regularity of the flux potential $\Psi^{A}$ in (5.40) then requires that $\operatorname{Im} \lambda^{A}=0$ at the boundary. Point (e) follows from point (1) of Section 6.1 for the flux field strengths, and their resulting equations (5.40) from which it is manifest that the form $\lambda_{A} \partial_{w} H$ must be smooth inside $\Sigma$.

Concretely, in view of the restriction to a boundary with a single connected component made in this paper for simplicity's sake, we shall parametrize $\Sigma$ by the upper half complex plane, whose boundary $\partial \Sigma$ is the real line. Equivalently, we may conformally map the upper half-plane to the unit-disk, whose boundary is the unit circle. 


\section{$7 \quad$ Regular locally asymptotically $A d S_{3} \times S^{3}$ solutions}

In this section, we shall produce the general form of the harmonic function $H$ and the holomorphic one-forms $\Lambda^{A}$ which will solve the topology and regularity conditions of Section 6 required for regular solutions with $N$ distinct $A d S_{3} \times S^{3}$ asymptotic regions. We shall parametrize $\Sigma$ by the upper half complex plane, and its boundary $\partial \Sigma$ by the real line.

\subsection{Solving the regularity conditions on $H$}

Positivity of the harmonic function $H$ inside $\Sigma$ by point (a) of Section 6.2 prohibits any poles in the interior of $\Sigma$. Since $H$ is real, all poles of $H$ must lie on the boundary $\partial \Sigma=\mathbf{R}$. We shall restrict attention to simple poles, as higher order poles may be reached by coalescing simple poles. Thus, we parametrize $H$ by $N$ simple poles $x_{n}$ on the real axis,

$$
H=\sum_{n=1}^{N} \frac{i c_{n}}{w-x_{n}}+\text { c.c. }
$$

The vanishing of $H$ on $\partial \Sigma$ by point (b) of Section 6.2 requires the residues $c_{n}$ to be real, while the positivity of $H$ inside $\Sigma$ by point (a) requires $c_{n}>0$. An alternative expression for $H$ is then given by,

$$
H=\operatorname{Im}(w) \sum_{n=1}^{N} \frac{2 c_{n}}{\left|w-x_{n}\right|^{2}}
$$

making the above enforced properties of $H$ manifest. In view of the relation $f_{1} f_{2}=H$, it is clear that one or both of the radius functions $f_{1}, f_{2}$ must diverge at the poles $x_{n}$, while the radii are finite elsewhere. We shall establish in detail later that each pole indeed corresponds to a distinct asymptotically $A d S_{3} \times S^{3}$ region.

\subsection{Solving the regularity conditions on $\Lambda^{A}$}

By point (e) of Section 6.2, the one-forms $\Lambda^{A}=\lambda^{A} \partial_{w} H$ must be holomorphic inside $\Sigma$, but may have poles on $\partial \Sigma$. Recasting the constraint relations (6.1) in terms of $\Lambda^{A}$,

$$
\begin{aligned}
& \Lambda \cdot \Lambda=2\left(\partial_{w} H\right)^{2} \\
& \Lambda \cdot \bar{\Lambda} \geq 2\left|\partial_{w} H\right|^{2}
\end{aligned}
$$

it is clear that $\Lambda^{A}$ must have double poles at each one of the poles $x_{n}$ of $H$. For simplicity, we shall assume here that $\Lambda^{A}$ has no other poles. Thus, we have the following form for $\Lambda^{A}$,

$$
\Lambda^{A}=\sum_{n=1}^{N}\left(\frac{-i \kappa_{n}^{A}}{\left(w-x_{n}\right)^{2}}+\frac{-i \mu_{n}^{A}}{w-x_{n}}\right)
$$


The residues $\kappa_{n}^{A}, \mu_{n}^{A}$ must be real. To establish this, we use (7.1) to see that $\partial_{w} H$ is purely imaginary for $w$ real, which with the help of point (d) of Section 6.2 implies,

$$
\operatorname{Im}\left(\lambda^{A}\right)=\operatorname{Re}\left(\Lambda^{A}\right)=0 \quad \text { on } \partial \Sigma
$$

The relation $\operatorname{Re}\left(\Lambda^{A}\right)=0$ readily implies that the residues $\kappa_{n}^{A}$ and $\mu_{n}^{A}$ must be real, as announced.

\subsection{Regularity at the poles of $\lambda^{A}$}

Unlike the one-forms $\Lambda^{A}$ which are holomorphic inside $\Sigma$, the scalars $\lambda^{A}$ are allowed to have poles in the interior of $\Sigma$, namely at all the points where $\partial_{w} H$ vanishes. These points come in complex conjugate pairs, and will be denoted by $w_{q}, \bar{w}_{q}$ with $\operatorname{Im}\left(w_{q}\right)>0$ and $q=1, \cdots, N-1$. Near a pole $w_{q}$ in $\Sigma$, the functions behave as follows,

$$
\lambda^{A}(w) \sim \frac{P_{q}^{A}}{w-w_{q}} \quad P_{q}^{A}=\frac{\Lambda^{A}\left(w_{q}\right)}{\partial_{w}^{2} H\left(w_{q}\right)}
$$

where $P_{q}^{A}$ are vectors of complex residues. Conditions (6.1) imply $P_{q} \cdot P_{q}=0$ and $P_{q} \cdot \bar{P}_{q}>0$. In the limit $w \rightarrow w_{q}$ we have $\lambda \cdot \bar{\lambda} \rightarrow+\infty$. In view of (5.25), we find two solutions for $|X|^{2}$, namely $|X|^{ \pm 2} \sim\left(P_{q} \cdot \bar{P}_{q}\right) /\left|w-w_{q}\right|^{2}$. In either case, the metric factors $f_{1}, f_{2}, \rho$ of $(5.37)$, the scalars $V^{+}{ }_{A}$ of (5.38), and the flux potentials of (5.40), are regular near $w_{q}$. The common phase to all $V^{+}{ }_{A}$ is a gauge artifact, and remains undetermined. We close by noting that at the poles $w_{q}$, the radius factors become equal, $f_{1}=f_{2}$, and multiply the Bertotti-Robinson metric on the space $A d S_{2} \times S^{2}$.

\subsection{Solving the constraints}

The harmonic function $H$ and the holomorphic one-forms $\Lambda^{A}$, provided by the parameterization of (7.1) and (7.4) respectively, must obey the constraints of (7.3) pointwise in order to produce genuine local supergravity solutions. In this section, we shall derive the relations between the positions of the poles $x_{n}$ and the residues $c_{n}, \kappa_{n}^{A}$, and $\mu_{n}^{A}$ implied by the first equation of (7.3). The explicit solution to these equations, as well as to the inequality relation of (7.3) will be constructed in Section 8.

The first relation of (7.3) produces an equation with poles at $x_{n}$ of order up to four, upon substituting $H$ of (7.1) and $\Lambda^{A}$ of (7.4). Matching the residues of the poles of orders $4,3,2$, and 1 requires the following relations,

$$
\kappa_{n} \cdot \kappa_{n}=2\left(c_{n}\right)^{2} \quad \kappa_{n} \cdot \mu_{n}=\mathcal{E}_{n}^{(2)}=\mathcal{E}_{n}^{(1)}=0
$$


for all $n=1, \cdots, N$, where we have defined the following combinations,

$$
\begin{aligned}
& \mathcal{E}_{n}^{(2)}=\mu_{n} \cdot \mu_{n}+2 \sum_{n^{\prime} \neq n} \frac{\kappa_{n} \cdot \mu_{n^{\prime}}}{x_{n}-x_{n^{\prime}}}-2 \sum_{n^{\prime} \neq n} \frac{2 c_{n} c_{n^{\prime}}-\kappa_{n} \cdot \kappa_{n^{\prime}}}{\left(x_{n}-x_{n^{\prime}}\right)^{2}} \\
& \mathcal{E}_{n}^{(1)}=\sum_{n^{\prime} \neq n} \frac{\mu_{n} \cdot \mu_{n^{\prime}}}{x_{n}-x_{n^{\prime}}}-\sum_{n^{\prime} \neq n} \frac{\kappa_{n} \cdot \mu_{n^{\prime}}-\mu_{n} \cdot \kappa_{n^{\prime}}}{\left(x_{n}-x_{n^{\prime}}\right)^{2}}+2 \sum_{n^{\prime} \neq n} \frac{2 c_{n} c_{n^{\prime}}-\kappa_{n} \cdot \kappa_{n^{\prime}}}{\left(x_{n}-x_{n^{\prime}}\right)^{3}}
\end{aligned}
$$

While the relations $\mathcal{E}_{n}^{(2)}=0$ are all independent from one another, one has the following constraints between the relations $\mathcal{E}_{n}^{(1)}=0$,

$$
\sum_{n}\left(x_{n}\right)^{k} \mathcal{E}_{n}^{(1)} \sim k \sum_{n}\left(x_{n}\right)^{k-1} \mathcal{E}_{n}^{(2)} \quad k=0,1,2
$$

Thus there are $N$ independent relations $\mathcal{E}_{n}^{(2)}=0$, and $N-3$ independent relations $\mathcal{E}_{n}^{(1)}=0$.

The second relation of (7.3) limits the allowed ranges of the parameters $x_{n}, c_{n}, \kappa_{n}^{A}, \mu_{n}^{A}$ of the solutions. It may be simplified by adding to it the real part of the first equation of (7.3), resulting in the following equivalent inequality,

$$
\operatorname{Re}(\Lambda) \cdot \operatorname{Re}(\Lambda) \geq 2\left(\operatorname{Re}\left(\partial_{w} H\right)\right)^{2}
$$

This inequality reduces to an equality on $\partial \Sigma$, since $\operatorname{Re}(\Lambda)=\operatorname{Re}\left(\partial_{w} H\right)=0$ for real $w$, but (7.10) must be obeyed strictly inside $\Sigma$. More properly, (7.10) should be viewed as a family of inequalities, parametrized by $w$. The fact that we shall be able to solve completely in Section 8 this intricate system is a highly non-trivial bonus of the BPS problem.

\subsection{Asymptotic behavior of the metric in $A d S_{3} \times S^{3}$ regions}

The asymptotic behavior of the metric factors $f_{1}, f_{2}, \rho$ near the poles $x_{n}$ is determined by the data $x_{n}, c_{n}, \kappa_{n}^{A}, \mu_{n}^{A}$, and will now be derived. Expanding $H$ near the poles $x_{n}$,

$$
H^{2} \sim \frac{4 c_{n}^{2} \operatorname{Im}(w)^{2}}{\left|w-x_{n}\right|^{4}} \quad\left|\partial_{w} H\right|^{2} \sim \frac{c_{n}^{2}}{\left|w-x_{n}\right|^{4}}
$$

we calculate the following asymptotic behaviors using (5.37) and we find,

$$
\begin{aligned}
\rho^{4} \sim \frac{\mu_{n} \cdot \mu_{n}}{8\left|w-x_{n}\right|^{4}} & f_{1}^{4} \sim \frac{8 c_{n}^{4}}{\mu_{n} \cdot \mu_{n}} \frac{1}{\left|w-x_{n}\right|^{4}} \\
f_{2}^{4} & \sim 2 \mu_{n} \cdot \mu_{n} \frac{\operatorname{Im}(w)^{4}}{\left|w-x_{n}\right|^{4}}
\end{aligned}
$$

Note that positivity of these metric factors requires

$$
\mu_{n} \cdot \mu_{n}>0
$$


a property that can also be extracted directly by enforcing (7.10) in the vicinity of $w \sim x_{n}$. In terms of polar coordinates near the pole defined by $w=x_{n}+r e^{i \theta}$, the full six-dimensional metric of the solution becomes,

$$
d s^{2} \sim \sqrt{2 \mu_{n} \cdot \mu_{n}}\left(\frac{d r^{2}}{r^{2}}+\frac{2 c_{n}^{2}}{\mu_{n} \cdot \mu_{n}} \frac{1}{r^{2}} d s_{A d S_{2}}^{2}+d \theta^{2}+\sin ^{2} \theta d s_{S^{2}}^{2}\right)
$$

We recognize the metric of $A d S_{3} \times S^{3}$ which confirms that the vicinity of each pole $x_{n}$ indeed produces an asymptotically $A d S_{3} \times S^{3}$ region.

\subsection{Calculation of the charges and conservation}

The Page charges in each asymptotic region were defined in (5.41), and may be obtained by taking the integral of $\Lambda^{A}$ along a small contour $\mathcal{I}_{n}$ which starts and ends on the boundary and encloses the pole at $w=x_{n}$,

$$
Q_{n}^{A}=i \sqrt{2} \pi \int_{\mathcal{I}_{n}} \Lambda^{A}+\text { c.c. }=2 \sqrt{2} \pi^{2} \mu_{n}^{A}
$$

Regularity of $\Lambda^{A}$ as differential one-forms when $w \rightarrow \infty$ requires,

$$
\sum_{n} \mu_{n}^{A}=0
$$

which corresponds to the condition of vanishing total Page charge of the solution.

\subsection{Attractor behavior of scalars fields in $A d S_{3} \times S^{3}$ regions}

The asymptotic behavior of the scalars $V^{(i, r)}{ }_{A}$ near the poles is also determined by the data $x_{n}, c_{n}, \kappa_{n}^{A}, \mu_{n}^{A}$, up to $S O(5) \times S O(m)$ gauge transformations. As was shown in (5.26), the components $V_{R}^{r}$ are determined, up to gauge transformations, by the components $V^{ \pm}{ }_{A}$, according to formulas given in (5.38). To compute their limits as $w \rightarrow x_{n}$, we first evaluate the limits of $\lambda^{A}$ and $X$. Since $|X| \rightarrow 1$ as $w \rightarrow x_{n}$, we will need to keep higher order terms, and assume that the phase of $X$ has a finite limit $X\left(x_{n}\right)$ as $w \rightarrow x_{n}$. To carry out these calculations, it will be useful to decompose the scalar fields as follows,

$$
V^{+}{ }_{A}\left(x_{n}\right)=X\left(x_{n}\right)\left(\frac{1}{2} \operatorname{Re} \lambda_{A}\left(x_{n}\right)+i \lim _{w \rightarrow x_{n}} \frac{\operatorname{Im} \lambda_{A}(w)}{|X(w)|^{2}-1}\right)
$$

To compute the term whose limit is indefinite, we determine the behavior of $|X|$ and $\operatorname{Im} \lambda_{A}$ as $w \rightarrow x_{n}$. We decompose the relevant functions as follows,

$$
\begin{aligned}
\partial_{w} H(w) & =\frac{-i}{\left(w-x_{n}\right)^{2}}\left(c_{n}+\left(w-x_{n}\right)^{2} \partial H_{n}(w)\right) \\
\Lambda^{A}(w) & =\frac{-i}{\left(w-x_{n}\right)^{2}}\left(\kappa_{n}^{A}+\left(w-x_{n}\right) \mu_{n}^{A}+\left(w-x_{n}\right)^{2} \Lambda_{n}^{A}(w)\right)
\end{aligned}
$$


where $\partial H_{n}(w)$ and $\Lambda_{n}^{A}(w)$ admit finite limits as $w \rightarrow x_{n}$. The resulting values $\partial H_{n}\left(x_{n}\right)$ and $\Lambda_{n}^{A}\left(x_{n}\right)$ are real. The equality $2\left(\partial_{w} H\right)^{2}=\Lambda \cdot \Lambda$, expanded to second order in $w-x_{n}$, imposes the familiar conditions for poles of orders 4, 3, and 2 of (7.7). Computing the combination $\lambda \cdot \bar{\lambda}$ to second order in $w-x_{n}$ we find,

$$
\lambda^{A}(w) \sim \frac{\kappa_{n}^{A}}{c_{n}}+\left(w-x_{n}\right) \frac{\mu_{n}^{A}}{c_{n}}+\left(w-x_{n}\right)^{2} \frac{\Lambda_{n}^{A}\left(x_{n}\right)}{c_{n}}-\left(w-x_{n}\right)^{2} \partial H_{n}\left(x_{n}\right) \frac{\kappa_{n}^{A}}{c_{n}^{2}}
$$

Using the equations of (7.7) to simplify the terms of order 0 and 1 , and to cancel the terms proportional to $\left(\operatorname{Re}(w)-x_{n}\right)^{2}$, we find to leading order,

$$
\begin{aligned}
\lambda \cdot \bar{\lambda} & \sim 2+2 \operatorname{Im}(w)^{2} \frac{\mu_{n} \cdot \mu_{n}}{c_{n}^{2}} \\
|X|^{2} & \sim 1 \pm \frac{\operatorname{Im}(w)}{c_{n}} \sqrt{2 \mu_{n} \cdot \mu_{n}}
\end{aligned}
$$

As a result, we find the following limiting values for the scalar fields,

$$
V^{+}{ }_{A}\left(x_{n}\right)=X\left(x_{n}\right)\left(\frac{\kappa_{n A}}{2 c_{n}}-i \frac{\mu_{n A}}{\sqrt{2 \mu_{n} \cdot \mu_{n}}}\right)
$$

Since the values of $\mu_{n}^{A}$ give the charges of the solution, we see that (ignoring the phase

artifact of $X\left(x_{n}\right)$ ), the imaginary parts of the scalars are fully attracted, and their values are uniquely determined in terms of the charges. The real parts depends on $N$ vectors $\kappa_{n} / c_{n}$, whose square equals 2 , and which are orthogonal to $\mu_{n}$ by (7.7).

\section{8 $\mathrm{SL}(2, \mathrm{R})$ transformations on equations and solutions}

Under $S L(2, \mathbf{R})$ automorphisms of $\Sigma$, which leave $\partial \Sigma$ invariant, the coordinate $w$ and the positions of the poles $x_{n}$ transform as follows,

$$
w \rightarrow w^{\prime}=\frac{\alpha w+\beta}{\gamma w+\delta} \quad x_{n} \rightarrow x_{n}^{\prime}=\frac{\alpha x_{n}+\beta}{\gamma x_{n}+\delta}
$$

where $\alpha, \beta, \gamma, \delta \in \mathbf{R}$ and $\alpha \delta-\beta \gamma=1$. Transforming also the residues at the poles of $\partial_{w} H$ and $\Lambda^{A}$ by $\mu_{n} \rightarrow \mu_{n}^{\prime}=\mu_{n}$ and,

$$
c_{n} \rightarrow c_{n}^{\prime}=\frac{c_{n}}{\left(\gamma x_{n}+\delta\right)^{2}} \quad \kappa_{n} \rightarrow \kappa_{n}^{\prime}=\frac{\kappa_{n}}{\left(\gamma x_{n}+\delta\right)^{2}}
$$

we obtain the following transformations rules,

$$
\begin{aligned}
H & \rightarrow H^{\prime}\left(w^{\prime}, \bar{w}^{\prime}\right)=H(w, \bar{w}) \\
\partial_{w} H & \rightarrow \partial_{w^{\prime}} H^{\prime}\left(w^{\prime}\right)=(\gamma w+\delta)^{2} \partial_{w} H(w) \\
\Lambda & \rightarrow \Lambda^{\prime}\left(w^{\prime}\right)=(\gamma w+\delta)^{2} \Lambda(w) \\
\lambda & \rightarrow \lambda^{\prime}\left(w^{\prime}\right)=\lambda(w)
\end{aligned}
$$


where the functions $H^{\prime}, \Lambda^{\prime}, \lambda^{\prime}$ are evaluated with the poles $x_{n}^{\prime}$ and the residues $c_{n}^{\prime}, \kappa_{n}^{\prime}, \mu_{n}^{\prime}$. As a result, the physical data of the solution, assembled in Section 5.4, have simple transformation properties. The functions $f_{1}, f_{2}, \rho^{2}|d w|^{2}$ of (5.37), the scalars fields $V$ of (5.39) and (5.26), and the flux potentials $\Phi^{A}, \Psi^{A}$ transform as scalars, possibly up to gauge transformations. Also, the fundamental relations of (7.3) defining the local solutions are invariant under $S L(2, \mathbf{R})$. Thus, the physical data of the solutions are invariant under $S L(2, \mathbf{R})$.

\subsection{Counting moduli of the solutions}

The enumeration of the basic parameters of the solutions as they enter $H$ and $\Lambda$, is as follows,

$$
\begin{array}{ll}
x_{n}, c_{n} & 2 N \\
\kappa_{n}, \mu_{n} & 2(m+2) N
\end{array}
$$

giving a total of $(2 m+6) N$ real parameters. The number of relations is as follows. The regularity conditions (7.16) provide $m+2$ relations; matching of the poles or orders 4,3 , and 2 each provide $N$ relations; matching of the first order poles only provides $N-3$ relations in view of the dependencies of (7.9), yielding a total of $4 N+m-1$ relations. Thus, the number of independent parameters which enter a solution is given by $(2 m+6) N-(4 N+m-1)=$ $(2 m+2) N-m+1$. The physical data are invariant under the $S L(2, \mathbf{R})$ transformations of Section 7.8, bringing the total number of physical parameters of the solution to,

$$
(2 m+2) N-m-2
$$

We shall now show that this counting precisely matches the counting of physical parameters.

\subsection{Counting physical parameters of the solutions}

On physical grounds, the solutions are expected to be determined by the charges of the threeform fields $G^{A}$ on the 3 -spheres in the asymptotic $A d S_{3} \times S^{3}$ regions, as well as by the values of the scalar fields there. The counting proceeds as follows. There are $m+2$ independent charge parameters in each asymptotic region, and one overall charge conservation relation, thus yielding a total of $(m+2)(N-1)$ charge parameters. There are $2 m$ scalar entries in each asymptotic region, but as is clear from (7.21), half of those are subject to the attractor mechanism and already fixed by the charges $\mu_{n}$. Thus, only the $m$ "un-attracted" scalars must be counted as independent parameters per asymptotic region, giving a total of $m N$ scalar parameters. This gels nicely with the fact that in each asymptotic region there are

$m+2$ entries $\kappa_{n}^{A} / c_{n}$ subject to the two constraints of (7.7). In conclusion, the number of physical parameters of the solution is given by $(m+2)(N-1)+m N=(2 m+2) N-m-2$, which matches perfectly with the counting of (7.26). 


\section{Numerical solutions, $N=2,3,4,5$}

In this section, we shall spell out the conditions on our solutions for the cases $N=2,3$, which includes the vacuum solution $A d S_{3} \times S^{3}$ and the Janus solutions found in [32]. Furthermore we will derive numerically examples of new solutions with $N=3,4,5$.

\subsection{Solutions with $N=2$}

For $N=2$ only two asymptotic $A d S_{3} \times S^{3}$ regions appear, with opposite charges, $\mu_{1}+\mu_{2}=0$, and the following general relations,

$$
\begin{array}{ll}
\kappa_{1} \cdot \kappa_{1}=2 c_{1}^{2} & \kappa_{1} \cdot \mu_{1}=0 \\
\kappa_{2} \cdot \kappa_{2}=2 c_{2}^{2} & \kappa_{2} \cdot \mu_{2}=0
\end{array}
$$

Using $S L(2, \mathbf{R})$ invariance to fix the poles at $x_{1}=0, x_{2}=1$, the relations of (7.7) reduce to,

$$
\mu_{1}^{2}=\mu_{2}^{2}=4 c_{1} c_{2}-2 \kappa_{1} \cdot \kappa_{2}
$$

while we find that the second condition of (7.3) is automatic. For $\kappa_{2}=-\kappa_{1}$, we have the vacuum solution $A d S_{3} \times S^{3}$, while for $\kappa_{2} \neq-\kappa_{1}$, we have the basic Janus solution of the $(0,4)$ six-dimensional supergravity [31].

\subsection{Solutions with $N=3$}

For $N=3$, there are three asymptotic $A d S_{3} \times S^{3}$ regions, with $\mu_{1}+\mu_{2}+\mu_{3}=0$, and,

$$
\begin{array}{ll}
\kappa_{n} \cdot \kappa_{n}=2 c_{n}^{2} & n=1,2,3 \\
\kappa_{n} \cdot \mu_{n}=0 &
\end{array}
$$

Using $S L(2, \mathbf{R})$ symmetry, we fix the poles at $x_{1}=-1, x_{2}=0, x_{3}=1$. The relations $\mathcal{E}_{n}^{(1)}=0$ of (7.7) follow from the relations $\mathcal{E}_{n}^{(2)}=0$, which reduce to the following equations,

$$
\begin{aligned}
0 & =\mu_{1}^{2}-\frac{1}{2} \kappa_{1} \cdot\left(\mu_{2}-\mu_{3}\right)+2 C_{12}+\frac{1}{2} C_{13} \\
0 & =\mu_{2}^{2}+2 \kappa_{2} \cdot\left(\mu_{1}-\mu_{3}\right)+2 C_{12}+2 C_{23} \\
0 & =\mu_{3}^{2}-\frac{1}{2} \kappa_{3} \cdot\left(\mu_{1}-\mu_{2}\right)+\frac{1}{2} C_{13}+2 C_{23}
\end{aligned}
$$

where we have used the abbreviation $C_{m n}=\kappa_{m} \cdot \kappa_{n}-2 c_{m} c_{n}$. Obeying equations (8.3) and (8.4) guarantees that we satisfy the first relation of (7.3). To satisfy the second relation of 

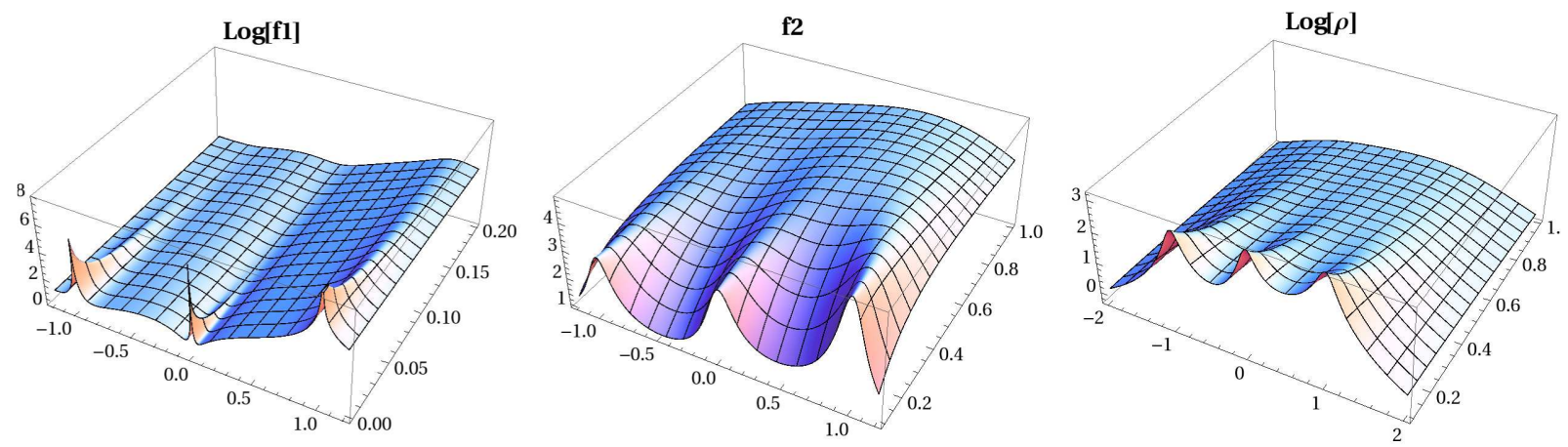

Figure 2: Metric factors for the upper half-plane parametrization of $\Sigma$
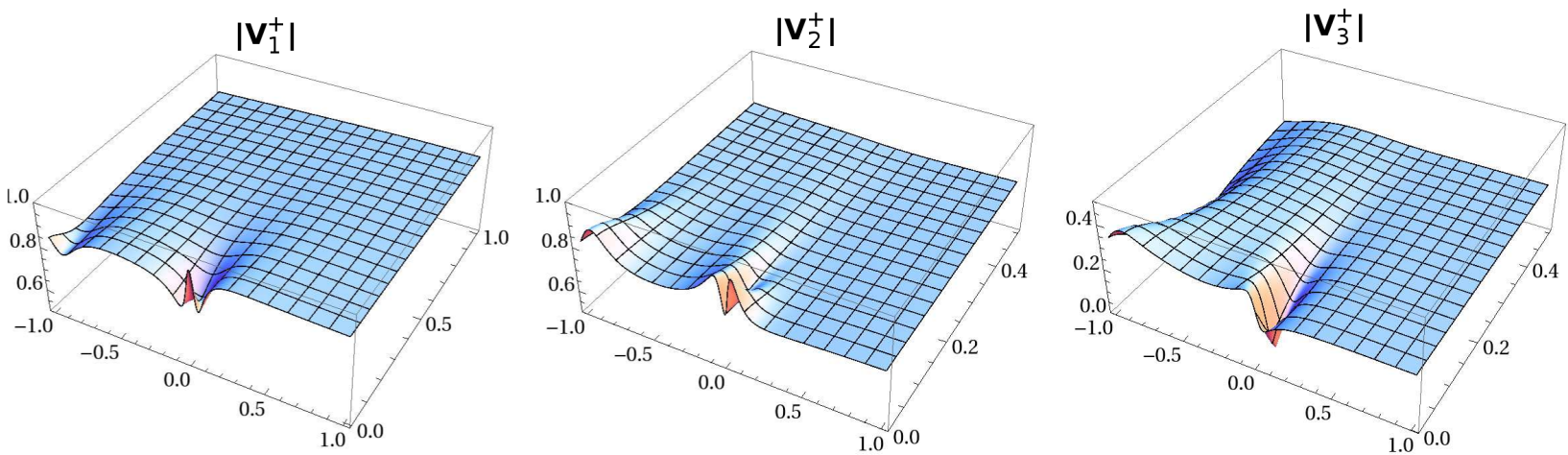

Figure 3: Scalars for the upper half-plane parametrization of $\Sigma$

(7.3) imposes further non-trivial conditions, which may be readily solved by numerical analysis, thus proving that, as soon as $N \geq 3$, solutions exist which are inequivalent to the solutions of [31] in that the scalar fields take values in a coset larger than the $S O(2,2) /(S O(2) \times S O(2))$ coset of [31]. Here we study numerically the case $N=3$ and with the scalars taking values in a $S O(2,4) /(S O(2) \times S O(4))$ coset space. In Figures 2 and 3, we present the numerical solution with poles brought back to the values $x_{1}=-1, x_{2}=0$, and $x_{3}=1$ by $S L(2, \mathbf{R})$ symmetry, and for the following data,

$$
\begin{aligned}
& \kappa_{1}=(-0.5621,-0.4099,-0.2461,-0.0706,-0.08979,-0.3586) \\
& \kappa_{2}=(-0.2835,-0.05616,-0.07683,-0.0174,-0.1350,0.1157) \\
& \kappa_{3}=(87.29,30.68,-23.11,40.00,-3.648,33.22) \\
& \mu_{1}=(3.681,-6.058,-0.8065,-0.2336,-0.5414,-0.4192) \\
& \mu_{2}=(1.557,-9.885,0.8671,-0.7100,-0.8268,0.4859)
\end{aligned}
$$

with $\mu_{3}=-\mu_{1}-\mu_{2}$, and the derived data $c_{1}=0.3753, c_{2}=0.1511$, and $c_{3}=51.52$. 


\subsection{Solutions with $Z_{N}$ symmetry}

An interesting subclass consists of solutions with cyclic $Z_{N}$ symmetry. To define these solutions, we map the upper half-plane coordinates $w, \bar{w}$ with $\operatorname{Im}(w)>0$ to the unit-disk coordinates $z, \bar{z}$ with $|z|=1$, by the following relations,

$$
\begin{aligned}
z & =\frac{1+i w}{1-i w} \\
z_{n} & =\frac{1+i x_{n}}{1-i x_{n}}
\end{aligned}
$$

Functions and forms transform as

$$
\begin{aligned}
\hat{H}(z, \bar{z}) & =H(w, \bar{w}) \\
\hat{\Lambda}^{A}(z) d z & =\Lambda^{A}(w) d w
\end{aligned}
$$

In the new coordinates, the functions $\hat{H}$ and $\hat{\Lambda}^{A}$ are found as follows,

$$
\begin{aligned}
\hat{H}(z, \bar{z}) & =\hat{H}_{0}-\frac{1}{2} \sum_{n=0}^{N-1} \frac{\left(z_{n}+1\right)^{2} c_{n}}{z-z_{n}}+\text { c.c. } \\
\hat{\Lambda}^{A}(z) & =\sum_{n=0}^{N-1}\left(-\frac{1}{2} \frac{\left(z_{n}+1\right)^{2} \kappa_{n}^{A}}{\left(z-z_{n}\right)^{2}}-\frac{i \mu_{n}^{A}}{z-z_{n}}\right)
\end{aligned}
$$

where the values $\mu_{n}^{A}$ are unchanged from the upper half-plane, and $\hat{H}_{0}$ is a constant such that $\hat{H}(z, \bar{z})=0$ for $|z|=1$.

The $Z_{N}$ transformations on the unit-disk are generated by,

$$
Z_{N}: \quad z \rightarrow \varepsilon z \quad \varepsilon=e^{2 \pi i / N}
$$

Invariance under $Z_{N}$ of the set of poles $z_{n}$ requires their values to be given by,

$$
z_{n}=\varepsilon^{n}
$$

up to an immaterial overall phase factor. The harmonic function $\hat{H}$ transforms as a scalar under $Z_{N}$, while the holomorphic form $\hat{\Lambda}^{A}$ transforms as a vector of $S O(2, m)$,

$$
\begin{aligned}
\hat{H}(\varepsilon z, \bar{\varepsilon} \bar{z}) & =\hat{H}(z, \bar{z}) \\
\hat{\Lambda}^{A}(\varepsilon z, \bar{\varepsilon} \bar{z}) d(\varepsilon z) & =E^{A}{ }_{B} \hat{\Lambda}^{B}(z) d z
\end{aligned}
$$

where the matrix $E$ provides an $S O(2, m)$-valued representation of the $Z_{N}$ transformation of (8.9), and thus obeys $E^{t} \eta E=\eta$ and $E^{N}=I$. Substituting the unit-disk expressions of 
(8.8) into the $Z_{N}$ covariance relations of (8.7), and identifying residues of like poles, gives the following relations,

$$
\begin{aligned}
c_{n} & =\frac{4 \varepsilon^{n} c_{0}}{\left(\varepsilon^{n}+1\right)^{2}} \\
\kappa_{n} & =\frac{4 \varepsilon^{n} E^{n} \kappa_{0}}{\left(\varepsilon^{n}+1\right)^{2}} \\
\mu_{n} & =E^{n} \mu_{0}
\end{aligned}
$$

Note that the $Z_{N}$ symmetry relation on $c_{n}$ is consistent with $c_{n}>0$. A convenient explicit representation for the matrix $E$ may be obtained, using an $S O(2, m)$ rotation, by changing to a basis in which $E$ assumes the form,

$$
E=\left(\begin{array}{ccccc}
d_{0} & 0 & 0 & \cdots & 0 \\
0 & d_{1} & 0 & \cdots & 0 \\
0 & 0 & d_{2} & \cdots & 0 \\
& & & \cdots & \\
0 & 0 & 0 & \cdots & d_{[m / 2]}
\end{array}\right)
$$

This form holds for $m$ even; for $m$ odd, an extra column and row need to be added whose only non-zero entry is 1 in the bottom right corner. The $2 \times 2$ blocks $d_{i}$ are given as follows,

$$
d_{i}=\left(\begin{array}{cc}
\cos \frac{2 \pi \nu_{i}}{N} & \sin \frac{2 \pi \nu_{i}}{N} \\
-\sin \frac{2 \pi \nu_{i}}{N} & \cos \frac{2 \pi \nu_{i}}{N}
\end{array}\right)
$$

The integers $\nu_{i}$ are defined $\bmod N$, and characterize the representation of $Z_{N}$ into $S O(2, m)$. 


\subsection{Numerical solutions with $Z_{4}$ and $Z_{5}$ symmetry}

In Figures 4 and 5, we present a numerical solution with $N=4$, effective scalar coset space $S O(2,4) /(S O(2) \times S O(4))$, and the values

$$
\nu_{1}=1 \quad \nu_{2}=1 \quad \nu_{3}=2
$$

The remaining data have been chosen randomly subject to the constraints (7.7-7.8),

$$
\begin{aligned}
& \mu_{0}=(-2.631,1.686,0.4054,-0.5013,-0.7915,-0.6094) \\
& \kappa_{0}=(0.3870,0.5423,-0.1557,-0.4702,0.2524,0.1260) \\
& c_{0}=0.2438
\end{aligned}
$$
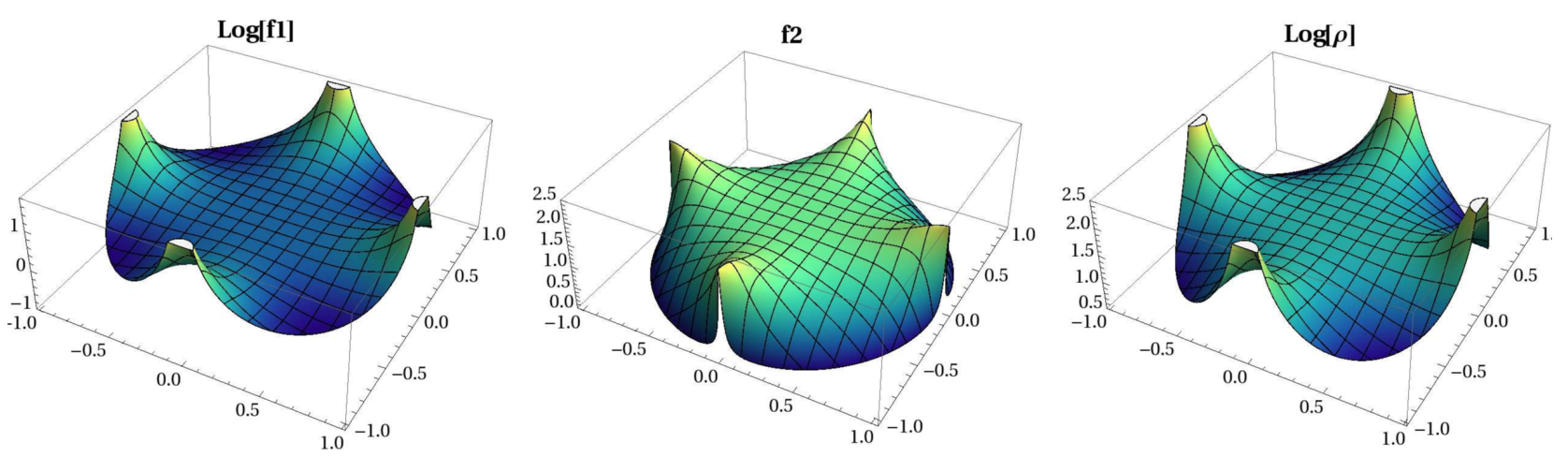

Figure 4: Metric factors for the $Z_{4}$-symmetric solution with data (8.15) and (8.16).
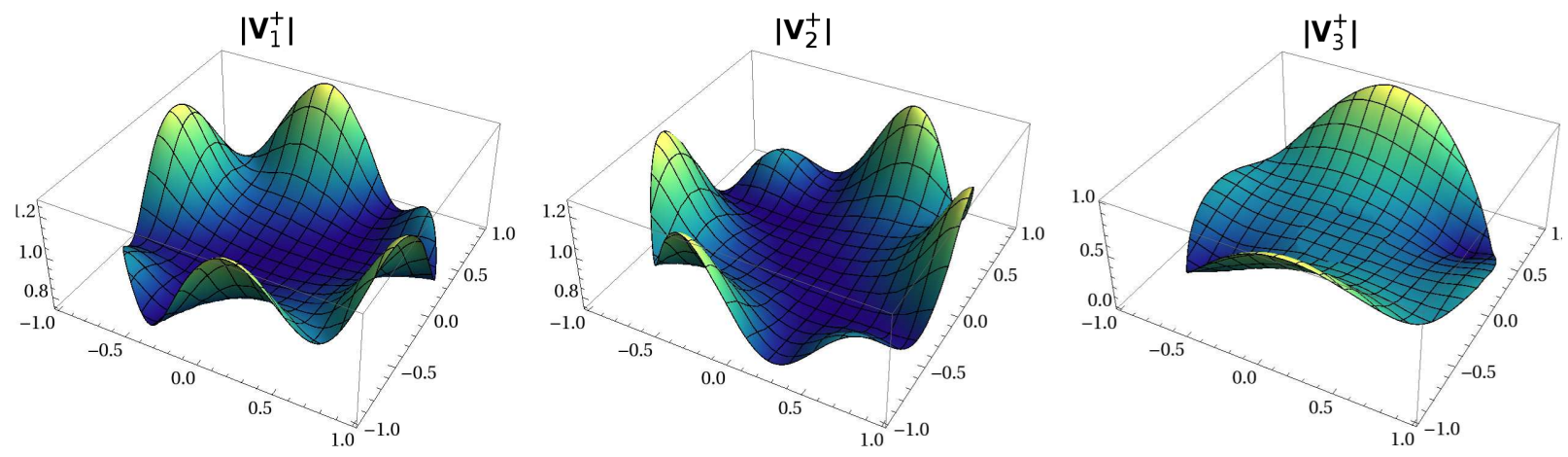

Figure 5: Scalar components for the $Z_{4}$-symmetric solution with data (8.15) and (8.16). 
In Figures 6 and 7 , we present a numerical solution with $N=5$, scalar coset space $S O(2,4) /(S O(2) \times S O(4))$ and the values

$$
\nu_{1}=1 \quad \nu_{2}=3 \quad \nu_{3}=2
$$

The remaining data have been chosen randomly subject to the constraints (7.7-7.8),

$$
\begin{aligned}
& \mu_{0}=(-2.481,2.273,-0.2239,-0.9870,0.9926,0.04811) \\
& \kappa_{0}=(0.3134,0.3310,0.2456,0.1550,0.1822,0.03752) \\
& c_{0}=0.2107
\end{aligned}
$$
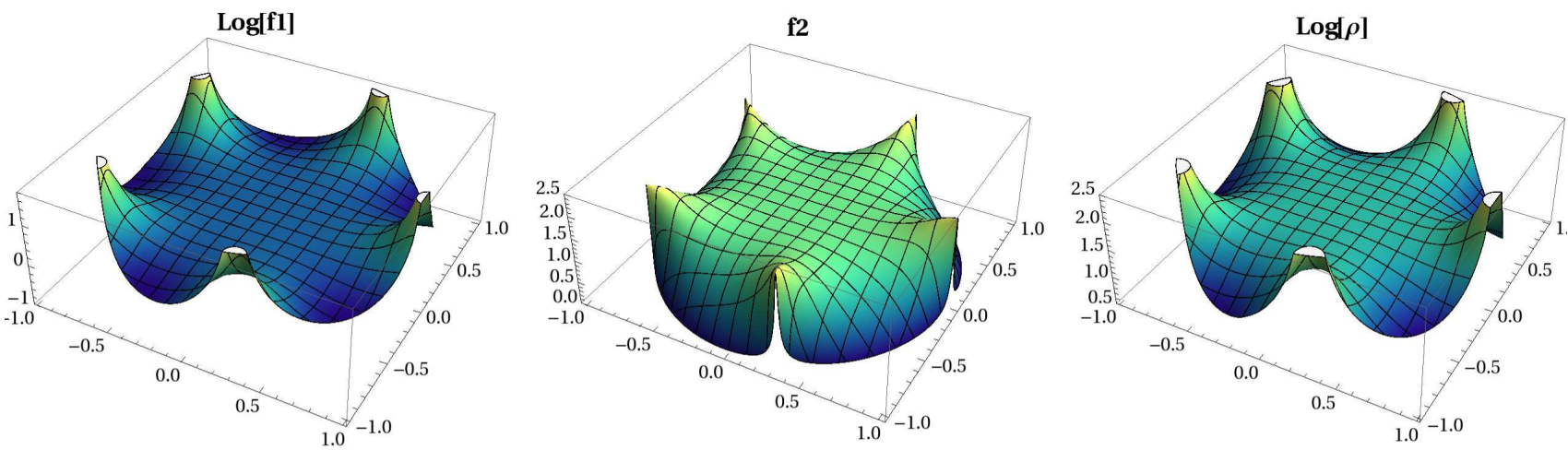

Figure 6: Metric factors for the $Z_{5}$-symmetric solution with data (8.17) (8.18).
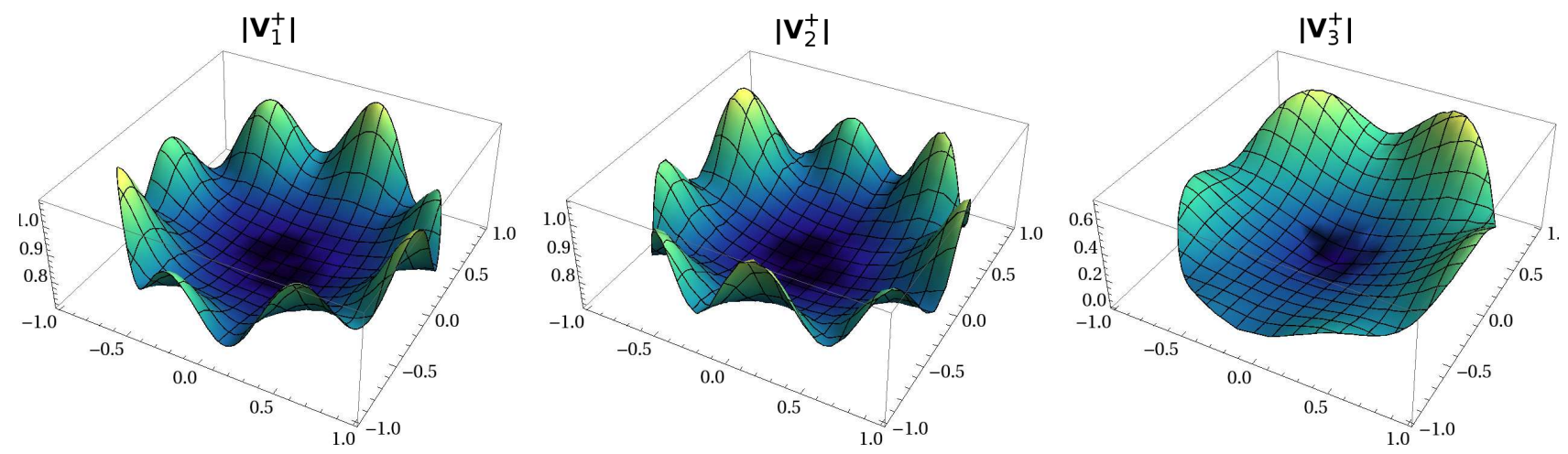

Figure 7: Scalar components for the $Z_{5}$-symmetric solution with data $(8.17)$ and (8.18). 


\section{Maximal families of regular solutions for all $m, N$}

In this section, we shall derive a general family of solutions with the maximal number $(2 m+2) N-m-2$ of free modular parameters. The corresponding half-BPS solutions, and their moduli space, may be parametrized explicitly and completely in terms data satisfying only simple linear inequalities. These data include a set of $2 N-2$ auxiliary poles, and associated residues, along with the mild technical assumption on these poles. The resulting families of regular solutions provide a generalization of the solutions of [31] to the case of general $m$, including new solutions for $m>2$.

\subsection{Light-cone like solution of the first constraint}

We begin by spelling out a light-cone like decomposition which will be at the core of the general and explicit parametrization of the families of solutions that we derive. The constraints, formulated in terms of $\lambda^{A}$, are as follows,

$$
\begin{aligned}
& 2=\lambda \cdot \lambda=\delta_{I J} \lambda^{I} \lambda^{J}-\delta_{R S} \lambda^{R} \lambda^{S} \\
& 2<\bar{\lambda} \cdot \lambda=\delta_{I J} \bar{\lambda}^{I} \lambda^{J}-\delta_{R S} \bar{\lambda}^{R} \lambda^{S}
\end{aligned}
$$

where $I, J=1,2, R, S=6, \cdots, m+5$. We choose one index $I$ of positive signature, and one index $R$ of negative signature of the metric $\eta$. By $S O(2) \times S O(m)$ covariance of the solution, these may be chose to be the indices 2 and 6 , without loss of generality. Accordingly, we define the inverse of the combination $\lambda^{2}-\lambda^{6}$,

$$
L^{6}=\frac{1}{\lambda^{2}-\lambda^{6}}
$$

and rescale the remaining fields as follows,

$$
L^{A}=\frac{L^{6}}{\sqrt{2}} \lambda^{A} \quad A=1,7,8, \cdots, m+5
$$

In terms of the functions $L^{A}$, the first constraint of (9.1) may be solved rationally, so that all $\lambda^{A}$ may be expressed in terms of the functions $L^{1}, L^{6}, L^{7} \cdots, L^{m+5}$. The counting works out, and the explicit formulas are given as follows,

$$
\begin{aligned}
& \lambda^{A}=\frac{\sqrt{2} L^{A}}{L^{6}} \quad A=1,7,8, \cdots, m+5 \\
& \lambda^{2}=\frac{1}{L^{6}}\left(+\frac{1}{2}-L^{1} L^{1}+\delta_{R S} L^{R} L^{S}\right) \\
& \lambda^{6}=\frac{1}{L^{6}}\left(-\frac{1}{2}-L^{1} L^{1}+\delta_{R S} L^{R} L^{S}\right)
\end{aligned}
$$




\subsection{Solving the second constraint}

In terms of the functions $L^{A}$, the second constraint of (9.1) simplifies considerably. To see this, we arrange the constraint in the following form,

$$
2<\bar{\lambda}^{1} \lambda^{1}+\frac{1}{2}\left(\bar{\lambda}^{2}+\bar{\lambda}^{6}\right)\left(\lambda^{2}-\lambda^{6}\right)+\frac{1}{2}\left(\bar{\lambda}^{2}-\bar{\lambda}^{6}\right)\left(\lambda^{2}+\lambda^{6}\right)-\sum_{A=7}^{m+5} \bar{\lambda}^{A} \lambda^{A}
$$

Eliminating $\lambda^{A}$ in favor of $L^{A}$ using (9.4), we obtain,

$$
0<-\left(L^{1}-\bar{L}^{1}\right)^{2}+\delta_{R S}\left(L^{R}-\bar{L}^{R}\right)\left(L^{S}-\bar{L}^{S}\right)
$$

where the summation over repeated indices now runs over $R, S=6,7, \cdots, m+5$. Introducing the following harmonic functions

$$
h^{A}=\operatorname{Im}\left(L^{A}\right) \quad A=1,6,7, \cdots, m+5
$$

equation (9.6), and thus the second constraint of (9.1), may be recast in the following form,

$$
\delta_{R S} h^{R} h^{S}<h^{1} h^{1}
$$

From the requirement that this inequality be obeyed strictly inside $\Sigma$, we deduce that $h_{1}$ must maintain the same sign inside $\Sigma$, which we shall take to be negative, $h_{1}<0$ inside $\Sigma$.

\subsection{Parametrization of solutions by "auxiliary poles"}

From the general expressions for $\partial_{w} H$ and $\Lambda^{A}$, given in (7.1) and (7.4), we see that each meromorphic function $\lambda^{A}$ is a ratio of two polynomials of degree $2 N-2$. Simple poles are allowed in $\lambda^{A}$ at the $2 N-2$ complex zeros $w_{q}, \bar{w}_{q}$ of $\partial_{w} H$, with $q=1, \cdots, N-1$. Each $\lambda^{A}$ has $2 N-2$ zeros. We shall denote the zeros of $\lambda^{2}-\lambda^{6}$ by $y_{p}$ where $p=1, \cdots, 2 N-2$. Hence, $L^{1}$ and $L^{R}$ have poles at the points $y_{p}$ and may be decomposed as follows,

$$
L^{A}(w)=\ell_{\infty}^{A}+\sum_{p=1}^{2 N-2} \frac{\ell_{p}^{A}}{w-y_{p}} \quad A=1, R
$$

For simplicity, we shall restrict here to the case where the poles $y_{p}$ are all simple.

Next, we show that the poles $y_{p}$ and residues $\ell_{p}^{A}$ have to be real. Since $h^{1}$ is a real harmonic function, it will assume both positive and negative values in any open neighborhood of each one of its poles. Thus, the condition $h^{1}<0$ inside $\Sigma$ prohibits any poles of $h^{1}$ from being complex, thereby forcing all poles $y_{p}$ to be real. Since $\lambda^{A}(w)$ must be real for real $w$, it follows that $L^{6}(w)$, and hence all the $L^{A}(w)$, must be real for $w \in \mathbf{R}$. In view of the decomposition 
of $(9.9)$, we see that real $L^{A}(w)$ for real $w$ requires $\ell_{\infty}^{A}$ and $\ell_{p}^{A}$ to be real. As a result, the harmonic functions take on simplified forms,

$$
h^{A}=-\operatorname{Im}(w) \sum_{p=1}^{2 N-2} \frac{\ell_{p}^{A}}{\left|w-y_{p}\right|^{2}} \quad A=1, R
$$

The absence of poles at the points $y_{p}$ in $\lambda^{2}$ and $\lambda^{6}$ (which were expressed in terms of $L^{A}$ in (9.4)) requires the following relation between the residues,

$$
\left(\ell_{p}^{1}\right)^{2}=\delta_{R S} \ell_{p}^{R} \ell_{p}^{S}
$$

Consistently with the result $h^{1}<0$ derived earlier, we choose the positive roots,

$$
\ell_{p}^{1}=\sqrt{\delta_{R S} \ell_{p}^{R} \ell_{p}^{S}}
$$

Condition (9.8) now becomes,

$$
0<\sum_{p, p^{\prime}=1}^{2 N-2} \frac{\ell_{p}^{1} \ell_{p^{\prime}}^{1}-\delta_{R S} \ell_{p}^{R} \ell_{p^{\prime}}^{S}}{\left|w-y_{p}\right|^{2}\left|w-y_{p^{\prime}}\right|^{2}}
$$

In view of (9.12) and the Schwarz inequality,

$$
0 \leq \ell_{p}^{1} \ell_{p^{\prime}}^{1}-\delta_{R S} \ell_{p}^{R} \ell_{p^{\prime}}^{S}
$$

for all $p, p^{\prime}=1, \cdots, 2 N-2$, the inequality $\leq$ in (9.13) follows automatically, and for all values of $\ell_{p}^{R}$, and all values of $y_{p}$.

For the strict inequality $<$ to hold in (9.13), as is required to have regular solutions, it suffices to have $0<\ell_{p}^{1} \ell_{p^{\prime}}^{1}-\delta_{R S} \ell_{p}^{R} \ell_{p^{\prime}}^{S}$ for a single pair of $p, p^{\prime}$. If the contrary were true, then the vectors $\ell_{p}^{R}$ would have to be parallel for all values of $p$ which leads to a trivial solution.

\subsection{Relating covariant data to auxiliary pole data}

Given a solution parametrized by the harmonic function $H$ and the auxiliary pole data $y_{p}$ and $\ell_{p}^{A}$, we seek to obtain formulas for the data $\kappa_{n}^{A}$ and $\mu_{n}^{A}$, which include the three-form Page charges. First, we relate the data to the values of $\lambda^{A}$ and its first derivative at $x_{n}$,

$$
\begin{aligned}
\kappa_{n}^{A} & =c_{n} \lambda^{A}\left(x_{n}\right) \\
\mu_{n}^{A} & =c_{n} \partial \lambda^{A}\left(x_{n}\right)
\end{aligned}
$$

Note that, in view of the relation $\lambda(w) \cdot \lambda(w)=2$, and its first derivative, the results of (9.15) automatically satisfy $\kappa_{n} \cdot \kappa_{n}=2 c_{n}^{2}$ as well as $\kappa_{n} \cdot \mu_{n}=0$. The values of $\lambda^{A}$ and its derivative may in turn be obtained from the relations (9.4). 


\subsection{Counting data and explicit parametrization of solutions}

The family of solutions may be parametrized - without leaving any unsolved equalities or inequalities - by the following data. We prescribe the positions of both sets of poles, $x_{n}$ and $y_{p}$ for $n=1, \cdots, N$ and $p=1, \cdots, 2 N-2$; give the residues $c_{n}$ for $n=1, \cdots, N$, and $\ell_{p}^{R}$ for $R=7,8, \cdots, m+5$; and prescribe the values at $w=\infty$, namely $\ell_{\infty}^{A}$ for $A=1,7,8, \cdots, m+5$. Finally, there is an overall real normalization factor $\ell_{\infty}^{6}$ for the function $L^{6}$. We will prove below that this set of data completely specifies the functions $H$ and $\lambda^{A}$, up to an overall redundancy by $S L(2, \mathbf{R})$ automorphisms of the upper half-plane. In this parametrization, we have the following counting of free parameters,

$\begin{array}{lll}x_{n} & N \\ c_{n} & N \\ y_{p} & 2 N-2 & \\ \ell_{p}^{R} & (m-1)(2 N-2) & R=7,8, \cdots, m+5 \\ \ell_{\infty}^{A} & m+1 & A=1,6,7,8, \cdots, m+5\end{array}$

The total number of data, including -3 degrees of freedom due to the action of $S L(2, \mathbf{R})$, is given by $(2 m+2) N-m-2$ in agreement with the results of $(7.26)$, which means that our family of solutions has maximal dimension.

To prove that the solutions are fixed completely by the parameters listed in (9.16), and that this parametrization solves all constraint equations, we proceed as follows. The data of (9.16) manifestly fix the functions $H$ and $L^{R}$ for $R=7,8, \cdots, m+5$. This leaves the functions $L^{1}$ and $L^{6}$ to be determined.

First, we determine $L^{6}$ by realizing that its poles are the zeros of $\lambda^{2}-\lambda^{6}$, which by definition are the points $y_{p}$ for $p=1, \cdots, 2 N-2$, while its zeros are the poles of $\lambda^{2}-\lambda^{6}$, which in turn are the zeros of $\partial_{w} H$. Thus, we can have the following formula for $L^{6}$,

$$
L^{6}=i \ell_{\infty}^{6} \frac{\prod_{n=1}^{N}\left(w-x_{n}\right)^{2}}{\prod_{p=1}^{2 N-2}\left(w-y_{p}\right)} \partial_{w} H
$$

where $\ell_{\infty}^{6}$ is a real overall normalization, which is being supplied by the parametrization of (9.16), and is equivalent to giving the value of $L^{6}$ at $w=\infty$. The pre-factor $i$ is required because $L^{6}$ is real-valued for $w$ real, while $\partial_{w} H$ is purely imaginary for $w$ real. From this formula, we extract

$$
\ell_{p}^{6}=i \ell_{\infty}^{6} \frac{\prod_{n=1}^{N}\left(y_{p}-x_{n}\right)^{2}}{\prod_{p^{\prime} \neq p}^{2 N-2}\left(y_{p}-y_{p^{\prime}}\right)} \partial_{w} H\left(y_{p}\right)
$$


for $p=1, \cdots, 2 N-2$. Second, using the relation (9.12), and the datum $\ell_{\infty}^{1}$ from the parametrization of (9.16), it is clear that also $L^{1}$ is known explicitly. Third, having now at our disposal the functions $H$ and $L^{A}$ with $A=1,6,7, \cdots, m+5$, we use (9.4) to derive all the $\lambda^{A}$ functions. Thus we have shown by explicit construction that, starting from the data of (9.16), the full solution is determined uniquely, up to inequalities on the parameters.

Finally, there is only a single group of inequalities to be satisfied,

$$
c_{n}>0 \quad n=1, \cdots, N
$$

All others, such as the fact that $L^{6}$ has only complex zeros, and that $h^{1}<0$ are automatically realized by our construction.

\subsection{Relation to the solutions of [31]}

The solutions to ten-dimensional Type IIB supergravity compactified on $K 3$ correspond to the case $m=2$ in our present work, since only dilaton, axion, four-form potential and $K 3$ metric factor produce scalars in the six-dimensional theory. The general solutions to the $K 3$ compactification of ten-dimensional Type IIB supergravity were obtained in [31] for all values of $N$, and provide solutions to the six-dimensional supergravity for general $N$. In this section, we spell out the correspondence.

To translate the solutions of [31] to the formulation used here, we may compare, for example, the expression for the metric $\rho^{2}$ on $\Sigma$, given by

$$
\rho^{4}=\frac{\left|\partial_{w} H\right|^{4}}{H^{2}}\left(\frac{(A+\bar{A}) K-B^{2}-\bar{B}^{2}}{B \bar{B}}-2\right)\left(\frac{(A+\bar{A}) K-B^{2}-\bar{B}^{2}}{B \bar{B}}+2\right)
$$

The harmonic function $H$ is the same in both formulations. Comparing with (5.37), we are led to making the following identification,

$$
\lambda \cdot \bar{\lambda}=\frac{(A+\bar{A}) K-B^{2}-\bar{B}^{2}}{B \bar{B}}
$$

The combination $\lambda \cdot \bar{\lambda}$ of (9.21) obeys the decomposition properties into a sum of holomorphic times anti-holomorphic functions, together with the purely holomorphic relation,

$$
\begin{aligned}
\lambda \cdot \bar{\lambda} & =\lambda_{1} \bar{\lambda}_{1}+\lambda_{2} \bar{\lambda}_{2}-\lambda_{6} \bar{\lambda}_{6}-\lambda_{7} \bar{\lambda}_{7} \\
2 & =\lambda_{1}^{2}+\lambda_{2}^{2}-\lambda_{6}^{2}-\lambda_{7}^{2}
\end{aligned}
$$

where the functions $\lambda_{A}$ are given by,

$$
\begin{aligned}
\lambda_{1} & =i \frac{A \mathcal{K}-B^{2}+1}{\sqrt{2} B} & \lambda_{6} & =i \frac{A \mathcal{K}-B^{2}-1}{\sqrt{2} B} \\
\lambda_{2} & =\frac{A+\mathcal{K}}{\sqrt{2} B} & \lambda_{7} & =\frac{A-\mathcal{K}}{\sqrt{2} B}
\end{aligned}
$$


where $\mathcal{K}$ is the holomorphic part of the harmonic function $K$ so that $K=\mathcal{K}+\overline{\mathcal{K}}$. Using the above relations together with (9.4), we can express the harmonic functions in [31] as

$$
\begin{aligned}
B & =i \sqrt{2} L^{6} \\
A+\bar{A} & =-2 \sqrt{2}\left(h^{1}+h^{7}\right) \\
K & =-2 \sqrt{2}\left(h^{1}-h^{7}\right)
\end{aligned}
$$

The reality and regularity conditions in terms of the functions $A, B, K, H$ were spelled out in $[31,32]$, and are given as follows,

1. The functions $H, A, B, K$ must be regular in the interior of $\Sigma$;

2. The functions $H, \operatorname{Re}(A), K$ cannot vanish in the interior of $\Sigma$;

3. All the zeros of $B$ and $\partial_{w} H$ must be common;

4. All poles of $A, B, \mathcal{K}$ must be simple, common, and located on $\partial \Sigma$, with related residues;

5. The inequality $\lambda \cdot \bar{\lambda}>2$ must hold in the interior of $\Sigma$;

6. The harmonic functions $H, \operatorname{Re}(A), \operatorname{Re}(B), K$ must obey vanishing Dirichlet boundary conditions on $\partial \Sigma$, while the harmonic functions $\operatorname{Im}(A)$ and $\operatorname{Im}(B)$ must satisfy Neumann boundary conditions on $\partial \Sigma$.

The five topology and regularity conditions of Section 6.2 on the locally holomorphic data for the solutions of this paper are seen to result from the above regularity assumptions on the solutions from Type IIB on K3. Specifically, condition (a) of Section 6.2 follows from conditions 1 and 2 above; condition (b) follows from 6; condition (c) is identical to 5; condition (d) follows from the explicit form (9.23) and the boundary conditions of 6 , which may also be read as $A, B, K$ are purely imaginary on $\partial \Sigma$; and condition (e) follows from the explicit form (9.23), the relation $\Lambda^{A}=\lambda^{A} \partial_{w} H$, and condition 3. Condition 4 above is equivalent to our earlier assumptions in (7.1) and (7.4) on the functional form of $H$ and $\Lambda^{A}$.

Finally, the requirements that $\operatorname{Re}(A)$ and $K$ cannot vanish inside $\Sigma$ are consequences of the relation (9.21) and condition 5. Indeed, at any point where $(A+\bar{A}) K=0$, we have $\lambda \cdot \bar{\lambda}=2-(B+\bar{B})^{2} /(B \bar{B}) \leq 2$ which violates condition 5 . 


\section{Discussion}

In this paper, we have explicitly constructed a general maximal family of global regular halfBPS solutions to $(0,4)$ six-dimensional supergravity with $m$ tensor multiplets. The spacetime of each one of these solutions consists of an $A d S_{2} \times S^{2}$ fibration over a Riemann surface $\Sigma$, and has $N$ distinct asymptotic $A d S_{3} \times S^{3}$ regions. In our construction, we have made the mild technical assumption that the one-forms $\Lambda^{A}$ have no poles on the real axis other than the poles of $H$. It would be interesting to see whether and how this restriction might be lifted, and if solutions with some singularities can still have a physical interpretation. Beyond this technical question, there are several directions in which the results presented here can be generalized and explored further.

We have focused on solutions of a particular six-dimensional supergravity, namely the $\left(n_{+}, n_{-}\right)=(0,4)$ theory. As discussed in Section 2.1.1 it is possible to embed our solutions into the $\left(n_{+}, n_{-}\right)=(4,4)$ theory. It is an open problem to apply the techniques of the present paper to six-dimensional supergravities with less supersymmetry and construct halfBPS string-junction solutions.

In future work, we plan to investigate the different degenerations of our family of solutions. In particular, a natural limit which should be analyzed in detail is the one where two or more poles of $H$ coalesce. This limit should lead to the factorization of the solution into two solutions with a lower number of asymptotic regions. In turn, a careful study of the factorization properties of the solutions may help relate some solutions to the near-horizon limit of dyonic string networks in six dimensions. Some of the bubbling solutions of Type IIB and M-theory have been related to brane webs in [36]. A related question is whether some limit of the solutions constructed in this paper can describe configurations of intersecting branes similar to the ones discussed in $[48,49,50]$ in a ten-dimensional setting.

The construction of globally regular solutions in Sections 7, 8, and 9 assumed that the Riemann surface $\Sigma$ has a single boundary component. This analysis can be generalized to Riemann surfaces with two or more boundary components along the lines of [32]. Such solutions should have novel and interesting features. First, additional homology three-spheres which are not associated with poles of the harmonic function $H$ will appear. These cycles can carry non-trivial anti-symmetric tensor flux. Second, in addition to degeneration limits where poles of $H$ coalesce, there are new degeneration limits where a boundary shrinks to zero size. In [32], this degeneration limit was found to produce new asymptotic regions of the form $A d S_{2} \times S^{2} \times S^{1} \times \mathbf{R}$.

The holographic interpretation of all Janus-type solutions is given by an interface CFT where different CFTs living on half-spaces are linked by a common interface world-line [51]. 
A proposal for the realization of a holographic dual to a boundary CFT as a limit of the Type IIB Janus solutions $[12,13]$ was recently set forth in $[52,53]$. It would be interesting to investigate whether a similar limit is possible for the solutions presented in this paper. A holographic realization of boundary CFT by introducing a brane which cuts off the AdS

bulk space and removes a second AdS half space was originally proposed in [54] and has recently been discussed in more detail in [55] .

In a recent paper [56] two of the authors calculated the boundary entropy of an interface conformal field theory using the holographic prescription developed in [57] and the solutions constructed in [31]. The solutions in the present paper are more general, and their parametrization via the harmonic function $H$ and the one-forms $\Lambda^{A}$ is more directly related to the physical charges and values of the scalars. Hence, to calculate the holographic boundary entropy of these new solutions along the lines of [56] and [57] seems a natural and promising enterprise. Finally, there are other observables, such as boundary correlation functions, which can be calculated holographically and compared to conformal field theory results. Such calculations would be extremely valuable in determining the exact map between holographic BPS Janus solutions and interface CFTs.

\section{Acknowledgements}

The work of ED, MG and YG was supported in part by NSF grant PHY-07-57702. The work of MC was supported in part by NSF grant PHY-08-55356. MC would like to thank Murat Günaydin, Radu Roiban, and Nicholas Warner for useful conversations and the Kavli Institute for Theoretical Physics for hospitality during the course of part of this work. MG would like to thank the Centro de ciencias de Benasque Pedro Pascual for hospitality while this paper was finalized. 


\section{A Notations and Conventions}

In this appendix, we spell out our core notations and conventions for the space-time and internal degrees of freedom of six-dimensional supergravity.

\section{A.1 Six-dimensional Minkowski space-time}

Throughout, we shall use the following index notations for six-dimensional space-time,

$$
\begin{aligned}
\mu, \nu=0, \cdots, 5 & \text { coordinate indices } \\
M, N=0, \cdots, 5 & \text { frame indices } \\
m, n=0,1 & \text { AdS } S_{2} \text { frame indices } \\
p, q=2,3 & S^{2} \text { frame indices } \\
a, b=4,5 & \Sigma \text { frame indices }
\end{aligned}
$$

The six-dimensional space-time metric has Minkowski signature. The Minkowski frame metric is chosen to be $\eta_{6}=\operatorname{diag}(-+++++)$ and will be used to raise and lower the frame indices $M, N, \cdots$. The completely anti-symmetric tensors $\epsilon$ acting on frame indices, in six and in two-dimensions, are normalized by,

$$
\epsilon_{012345}=\epsilon_{45}=1
$$

Our conventions for differential forms are the standard ones, namely the $p$-form $T^{(p)}$ associated with a totally anti-symmetric tensor $T_{\mu_{1} \cdots \mu_{p}}^{(p)}$ is normalized by

$$
T^{(p)}=\frac{1}{p !} T_{\mu_{1} \cdots \mu_{p}}^{(p)} d x^{\mu_{1}} \wedge \cdots \wedge d x^{\mu_{p}}
$$

The frame one-forms are defined by $e^{M}=e^{M}{ }_{\mu} d x^{\mu}$. Throughout, we shall use the notation $e^{M_{1} \cdots M_{p}}=e^{M_{1}} \wedge \cdots \wedge e^{M_{p}}$ so that the symbol $e^{012345}$ stands for the space-time volume form. The $p$-form $T^{(p)}$ may be expressed alternatively in frame indices,

$$
T^{(p)}=\frac{1}{p !} T_{M_{1} \cdots M_{p}}^{(p)} e^{M_{1} \cdots M_{p}}
$$

The Poincaré dual of a $p$-form $T^{(p)}$ may be defined equivalently by the following relations,

$$
\begin{aligned}
\left(* T^{(p)}\right)^{M_{1} \cdots M_{6-p}} & =\frac{1}{p !} \epsilon^{M_{1} \cdots M_{6-p} N_{1} \cdots N_{p}} T_{N_{1} \cdots N_{p}}^{(p)} \\
S^{(p)} \wedge * T^{(p)} & =\frac{(-1)^{p(6-p)}}{p !} S^{(p) N_{1} \cdots N_{p}} T_{N_{1} \cdots N_{p}}^{(p)} e^{012345}
\end{aligned}
$$

where the second line is to hold for all $p$-forms $S^{(p)}$. 


\section{A.2 $S O(5, m) /(S O(5) \times S O(m))$ coset space notations}

We use the following index notations for the coset space $S O(5, m) / S O(5) \times S O(m)$ variables,

$$
\begin{array}{cc}
A, B=1, \cdots m+5 & S O(5, m) \text { defining representation indices } \\
& A=(I, R) \quad I, J=1, \cdots, 5 \\
& B=(J, S) \quad R, S=6, \cdots, m+5 \\
i, j=1, \cdots, 5 & S O(5) \text { defining representation indices } \\
r, s=1, \cdots, n & S O(m) \text { defining representation indices } \\
\alpha, \beta=1, \cdots, 4 & S O(5) \text { spinor representation indices }
\end{array}
$$

\section{A.3 $S O(1,5)$-gamma matrices}

The $\gamma$-matrices in six dimensions are defined by $\left\{\gamma^{M}, \gamma^{N}\right\}=2 \eta_{6}^{M N}$, and we choose the basis,

$$
\begin{aligned}
i \gamma^{0} & =\sigma_{2} \otimes I_{2} \otimes I_{2} & & \gamma^{3}=\sigma_{3} \otimes \sigma_{2} \otimes I_{2} \\
\gamma^{1} & =\sigma_{1} \otimes I_{2} \otimes I_{2} & & \gamma^{4}=\sigma_{3} \otimes \sigma_{3} \otimes \sigma_{1} \\
\gamma^{2} & =\sigma_{3} \otimes \sigma_{1} \otimes I_{2} & & \gamma^{5}=\sigma_{3} \otimes \sigma_{3} \otimes \sigma_{2}
\end{aligned}
$$

Throughout, $S O(1,5)$ spinor indices will be suppressed. The chirality matrix $\gamma^{7}$, defined by,

$$
\gamma^{7}=\gamma^{012345}=\sigma_{3} \otimes \sigma_{3} \otimes \sigma_{3}
$$

obeys $\left(\gamma^{7}\right)^{2}=I$, as well as the following relations,

$$
\begin{aligned}
\gamma^{M N P Q R S} & =-\gamma^{7} \epsilon^{M N P Q R S} \\
\gamma^{M N P} \gamma^{7} & =-\frac{1}{3 !} \epsilon^{M N P Q R S} \gamma_{Q R S}
\end{aligned}
$$

The complex conjugation matrix $\mathcal{B}$ is defined to obey,

$$
\left(\gamma^{M}\right)^{*}=\mathcal{B} \gamma^{M} \mathcal{B}^{-1}
$$

and is given in the representation (A.7) by,

$$
\mathcal{B}=I_{2} \otimes \sigma_{1} \otimes \sigma_{2}
$$

The matrix $\mathcal{B}$ obeys $\mathcal{B B}^{*}=-I_{8}$; it commutes with $\gamma^{7}$.

Representing the six-dimensional space-time by the warped product $A d S_{2} \times S^{2} \times \Sigma$, it is natural to restrict the Dirac matrices to these two-dimensional factors. Denoting the 
corresponding reduced matrices by $\tilde{\gamma}^{M}$, and their associated reduced chirality matrices respectively by $\tilde{\gamma}_{(1)}, \tilde{\gamma}_{(2)}$, and $\tilde{\gamma}_{(3)}$, we have the following induced representations,

$$
\begin{array}{r}
\tilde{\gamma}^{1}=\tilde{\gamma}^{2}=\tilde{\gamma}^{4}=\sigma_{1} \\
i \tilde{\gamma}^{0}=\tilde{\gamma}^{3}=\tilde{\gamma}^{5}=\sigma_{2} \\
\tilde{\gamma}_{(1)}=\tilde{\gamma}_{(2)}=\tilde{\gamma}_{(3)}=\sigma_{3}
\end{array}
$$

In this representation, the chirality matrix $\gamma^{7}$ and the complex conjugation matrix $\mathcal{B}$ become,

$$
\begin{aligned}
\gamma^{7} & =\tilde{\gamma}_{(1)} \otimes \tilde{\gamma}_{(2)} \otimes \tilde{\gamma}_{(3)} \\
\mathcal{B} & =B_{(1)} \otimes B_{(2)} \otimes B_{(3)}
\end{aligned}
$$

The complex conjugation matrices in each subspace may be chosen as follows,

$$
\begin{array}{rll}
\left(\tilde{\gamma}^{m}\right)^{*}=+B_{(1)} \tilde{\gamma}^{m} B_{(1)}^{-1} & \left(B_{(1)}\right)^{*} B_{(1)}=+I_{2} & B_{(1)}=I_{2} \\
\left(\tilde{\gamma}^{p}\right)^{*}=+B_{(2)} \tilde{\gamma}^{p} B_{(2)}^{-1} & \left(B_{(2)}\right)^{*} B_{(2)}=+I_{2} & B_{(2)}=\sigma_{1} \\
\left(\tilde{\gamma}^{a}\right)^{*}=-B_{(3)} \tilde{\gamma}^{a} B_{(3)}^{-1} & \left(B_{(3)}\right)^{*} B_{(3)}=-I_{2} & B_{(3)}=\sigma_{2}
\end{array}
$$

An alternative representation is obtained by reversing the roles of $B_{(2)}$ and $B_{(3)}$.

\section{A.4 $S O(5)$-gamma matrices}

The internal $S O(5)=U S p(4)$ structure of six-dimensional supergravity involves $\Gamma$-matrices, defined by $\left\{\Gamma^{i}, \Gamma^{j}\right\}=2 \delta^{i j}$, where $\delta^{i j}$ in the $S O(5)$ frame metric. We use the following basis,

$$
\begin{array}{ll}
\Gamma^{1}=\sigma_{1} \otimes I_{2} & \Gamma^{3}=\sigma_{3} \otimes \sigma_{1} \\
\Gamma^{2}=\sigma_{2} \otimes I_{2} & \Gamma^{4}=\sigma_{3} \otimes \sigma_{2} \\
& \Gamma^{5}=\sigma_{3} \otimes \sigma_{3}
\end{array}
$$

The charge conjugation matrix $\mathcal{C}$ is defined by $\left(\Gamma^{i}\right)^{t}=\left(\Gamma^{i}\right)^{*}=\mathcal{C} \Gamma^{i} \mathcal{C}^{-1}$. In the basis of (A.15), the matrix $\mathcal{C}$ is given by,

$$
\mathcal{C}=\sigma_{1} \otimes \sigma_{2}
$$

The following basis-independent properties of $\mathcal{C}$ hold,

$$
\mathcal{C}+\mathcal{C}^{t}=\mathcal{C} \Gamma^{i}+\left(\mathcal{C} \Gamma^{i}\right)^{t}=\mathcal{C} \Gamma^{i j}-\left(\mathcal{C} \Gamma^{i j}\right)^{t}=0
$$

The anti-symmetry property will be crucial for the reduction of the BPS equations. 


\section{A.5 Choice of a spinor basis in $A d S_{2} \times S^{2} \times \Sigma$}

In this section we briefly review the definitions of the Killing spinors on $A d S_{2}$ and $S^{2}$, an explicit construction of these spinors can be found for example in [8, 12]. We choose the Killing spinor equation for $A d S_{2}$ which gives a real equation in the basis of $\gamma$-matrices that we have chosen in (A.12). This Killing spinor equation is given by

$$
\nabla_{m} \chi_{(1)}^{\eta}-\frac{\eta}{2} \tilde{\gamma}_{m} \chi_{(1)}^{\eta}=0
$$

Integrability demands that $\eta= \pm 1$ and there are two linearly independent solutions for each choice of $\eta$. The Killing spinor equation on $S^{2}$ is chosen as

$$
\nabla_{p} \chi_{(2), a}^{\eta}-\frac{i \eta}{2} \tilde{\gamma}_{p} \chi_{(2), a}^{\eta}=0
$$

where $\eta= \pm 1$ from the integrability condition. For each choice of $\eta$ there are two linear independent solutions denoted by the subscript $a$. These two solutions are related by complex conjugation. A suitable basis of invariant spinors on the full six-dimensional space-time $A d S_{2} \times S^{2} \times \Sigma$ is then obtained as follows,

$$
\chi_{a}^{\eta_{1}, \eta_{2}, \eta_{3}} \equiv \chi_{(1)}^{\eta_{1}} \otimes \chi_{(2) a}^{\eta_{2}} \otimes \chi_{(3)}^{\eta_{3}} \quad a=1,2
$$

The action of chirality is as follows,

$$
\begin{aligned}
\gamma_{(1)} \chi^{\eta_{1}, \eta_{2}, \eta_{3}} & =\chi^{-\eta_{1}, \eta_{2}, \eta_{3}} \\
\gamma_{(2)} \chi^{\eta_{1}, \eta_{2}, \eta_{3}} & =\chi^{\eta_{1},-\eta_{2}, \eta_{3}} \\
\gamma_{(3)} \chi^{\eta_{1}, \eta_{2}, \eta_{3}} & =\eta_{3} \chi^{\eta_{1}, \eta_{2}, \eta_{3}}
\end{aligned}
$$

The action of complex conjugation is also well-defined in this basis,

$$
\begin{aligned}
& \mathcal{B}^{-1}\left(\chi_{1}^{\eta_{1}, \eta_{2}, \eta_{3}}\right)^{*}=-i \eta_{2} \eta_{3} \chi_{2}^{\eta_{1},-\eta_{2},-\eta_{3}} \\
& \mathcal{B}^{-1}\left(\chi_{2}^{\eta_{1}, \eta_{2}, \eta_{3}}\right)^{*}=+i \eta_{2} \eta_{3} \chi_{1}^{\eta_{1},-\eta_{2},-\eta_{3}}
\end{aligned}
$$

Finally, we recall the action of the covariant derivatives,

$$
\begin{array}{lll}
\left(\hat{\nabla}_{m}-\frac{1}{2} \eta_{1} \tilde{\gamma}_{m} \otimes I \otimes I\right) \chi_{a}^{\eta_{1}, \eta_{2}, \eta_{3}}=0 & m=0,1 \\
\left(\hat{\nabla}_{p}-\frac{i}{2} \eta_{2} I \otimes \tilde{\gamma}_{p} \otimes I\right) \chi_{a}^{\eta_{1}, \eta_{2}, \eta_{3}}=0 & p=2,3
\end{array}
$$

A general spinor $\varepsilon^{\alpha}$ (with $S O(5)$-spinor index $\alpha$, and $S O(1,5)$ index suppressed), may now be decomposed onto the basis vectors $\chi$ as follows,

$$
\varepsilon^{\alpha}=\sum_{\eta_{1}, \eta_{2}, \eta_{3}, a} \chi_{a}^{\eta_{1}, \eta_{2}, \eta_{3}} \otimes \zeta_{\eta_{1}, \eta_{2}, \eta_{3}, a}^{\alpha}
$$


It is clear from the complex conjugation relation, however, that the spinor $\chi_{2}$ is proportional to $\mathcal{B}^{-1}\left(\chi_{1}\right)^{*}$. Thus, we may equivalently use the following decomposition,

$$
\varepsilon^{\alpha}=\sum_{\eta_{1}, \eta_{2}, \eta_{3}}\left(\chi_{1}^{\eta_{1}, \eta_{2}, \eta_{3}} \otimes \zeta_{\eta_{1}, \eta_{2}, \eta_{3}}^{\alpha}+\mathcal{B}^{-1}\left(\chi_{1}^{\eta_{1}, \eta_{2}, \eta_{3}}\right)^{*} \otimes \hat{\zeta}_{\eta_{1}, \eta_{2}, \eta_{3}}^{\alpha}\right)
$$

Note that the $A d S_{2}$ spinor is doubly degenerate, so that each solution of the type given above generates two supersymmetries.

\section{B Reducing the BPS equations}

In this appendix, we present the details of the reduction of the BPS equations. We first derive some results that will be used later. Notice that the $\eta_{3}$ index is virtually the $\sigma^{3}$ basis for the space $\Sigma$, namely,

$$
\chi^{\eta_{1}, \eta_{2},+}=\chi^{\eta_{1}, \eta_{2}} \otimes\left(\begin{array}{l}
1 \\
0
\end{array}\right) \quad \chi^{\eta_{1}, \eta_{2},-}=\chi^{\eta_{1}, \eta_{2}} \otimes\left(\begin{array}{l}
0 \\
1
\end{array}\right)
$$

we have the following relation,

$$
\gamma^{a} \chi^{\eta_{1}, \eta_{2}, \eta_{3}}=\sigma^{3} \otimes \sigma^{3} \otimes \sigma^{a} \chi^{\eta_{1}, \eta_{2}, \eta_{3}}=\chi^{-\eta_{1},-\eta_{2}, \eta_{3}^{\prime}}\left(\sigma^{a}\right)_{\eta_{3}^{\prime} \eta_{3}}
$$

and we can translate the action of $\sigma^{a}$ on $\chi$ to the action on $\zeta$ in the following way,

$$
\begin{aligned}
\sigma^{3} \otimes \sigma^{3} \otimes \sigma^{a}\left(\chi^{\eta_{1}, \eta_{2}, \eta_{3}} \zeta_{\eta_{1}, \eta_{2}, \eta_{3}}\right) & =\chi^{-\eta_{1},-\eta_{2}, \eta_{3}^{\prime}}\left(\sigma^{a}\right)_{\eta_{3}^{\prime} \eta_{3}} \zeta_{\eta_{1}, \eta_{2}, \eta_{3}} \\
& =\chi^{\eta_{1}, \eta_{2}, \eta_{3}}\left(\tau^{(11 a)} \zeta\right)_{\eta_{1}, \eta_{2}, \eta_{3}}
\end{aligned}
$$

where we make use of the $\tau$-matrix notation. Similarly for the charge conjugate basis,

$$
\begin{aligned}
\sigma^{3} \otimes \sigma^{3} \otimes \sigma^{a}\left(\left(\chi^{c}\right)^{\eta_{1}, \eta_{2}, \eta_{3}} \mathcal{C}_{\beta}^{\alpha} \zeta_{\eta_{1}, \eta_{2}, \eta_{3}}^{\beta *}\right) & =\mathcal{B}\left(\sigma^{3} \otimes \sigma^{3} \otimes \sigma^{a} \chi^{\eta_{1}, \eta_{2}, \eta_{3}}\right)^{*} \mathcal{C}_{\beta}^{\alpha} \zeta_{\eta_{1}, \eta_{2}, \eta_{3}}^{\beta *} \\
& =\left(\chi^{c}\right)^{\eta_{1}, \eta_{2}, \eta_{3}} \mathcal{C}_{\beta}^{\alpha}\left(\tau^{(11 a)} \zeta^{\beta}\right)_{\eta_{1}, \eta_{2}, \eta_{3}}^{*}
\end{aligned}
$$

As argued above in the previous section, the $\left(\chi^{c}\right)^{\eta_{1}, \eta_{2}, \eta_{3}}$ term in $\varepsilon^{\alpha}$ spinor will just give the complex conjugate of the $\chi^{\eta_{1}, \eta_{2}, \eta_{3}}$ term equations, hence below we shall focus on the $\chi^{\eta_{1}, \eta_{2}, \eta_{3}}$ term, and omit its conjugate terms.

\section{B.1 Reduction of the gravitino BPS equation}

The gravitino equation may be expressed as follows,

$$
d \varepsilon+\omega \varepsilon+\phi \varepsilon+\theta \varepsilon=0
$$


where the ingredients are defined as follows,

$$
\begin{aligned}
(\omega \varepsilon)^{\alpha} & =\frac{1}{4} \omega_{M N} \gamma^{M N} \varepsilon^{\alpha} \\
(\phi \varepsilon)^{\alpha} & =-\frac{1}{4} Q^{i j}\left(\Gamma_{i j}\right)_{\beta}^{\alpha} \varepsilon^{\beta} \\
(\theta \varepsilon)^{\alpha} & =-\frac{1}{4} H_{M N P}^{i} e^{M} \gamma^{N P}\left(\Gamma^{i}\right)^{\alpha}{ }_{\beta} \varepsilon^{\beta}
\end{aligned}
$$

To calculate the contribution $d \varepsilon+\omega \varepsilon$, we evaluate the connection. The non-vanishing spin connection components are $\omega^{a}{ }_{b}$ and

$$
\begin{aligned}
\omega^{m}{ }_{n} & =\hat{\omega}^{m}{ }_{n} & \omega^{m}{ }_{a} & =e^{m} \frac{D_{a} f_{1}}{f_{1}} \\
\omega^{p}{ }^{p} & =\hat{\omega}^{p}{ }_{q} & \omega^{p}{ }_{a} & =e^{p} \frac{D_{a} f_{2}}{f_{2}}
\end{aligned}
$$

The hats refer to the canonical connections on $A d S_{2}$ and $S^{2}$. In components along respectively $e^{m}, e^{p}$, and $e^{a}$, the contribution $d \varepsilon+\omega \varepsilon$ projects as follows,

$$
\begin{array}{ll}
(m) & \hat{\nabla}_{m} \varepsilon^{\alpha}+\frac{1}{2} \frac{D_{a} f_{1}}{f_{1}} \gamma_{m} \gamma^{a} \varepsilon^{\alpha} \\
(p) & \hat{\nabla}_{i} \varepsilon^{\alpha}+\frac{1}{2} \frac{D_{a} f_{2}}{f_{2}} \gamma_{i} \gamma^{a} \varepsilon^{\alpha} \\
(a) & \nabla_{a} \varepsilon^{\alpha}
\end{array}
$$

where the hats on the covariant derivatives indicates that only the connection along respectively $A d S_{2}$, and $S^{2}$ have been included. Using the Killing spinor equations (A.18) and (A.19) to eliminate the hatted covariant derivatives, and combining the terms, we obtain,

$$
\begin{array}{ll}
(m) & \gamma_{m} \sum_{\eta_{1}, \eta_{2}, \eta_{3}} \chi^{\eta_{1}, \eta_{2}, \eta_{3}} \otimes\left[\frac{1}{2 f_{1}} \tau^{(300)} \zeta_{\eta_{1}, \eta_{2}, \eta_{3}}^{\alpha}+\frac{D_{a} f_{1}}{2 f_{1}}\left(\tau^{(11 a)} \zeta^{\alpha}\right)_{\eta_{1}, \eta_{2}, \eta_{3}}\right] \\
(p) & \gamma_{p} \sum_{\eta_{1}, \eta_{2}, \eta_{3}} \chi^{\eta_{1}, \eta_{2}, \eta_{3}} \otimes\left[\frac{i}{2 f_{2}} \tau^{(130)} \zeta_{\eta_{1}, \eta_{2}, \eta_{3}}^{\alpha}+\frac{D_{a} f_{2}}{2 f_{2}}\left(\tau^{(11 a)} \zeta^{\alpha}\right)_{\eta_{1}, \eta_{2}, \eta_{3}}\right]
\end{array}
$$

where we have pulled a factor of $\gamma_{M}$ out front for equation (m) and (p). To calculate the term $\phi \varepsilon$, we observe that the only non-vanishing direction is along $e^{a}$,

$$
\text { (a) } \quad-\frac{1}{4} \sum_{\eta_{1}, \eta_{2}, \eta_{3}} \chi^{\eta_{1}, \eta_{2}, \eta_{3}} \otimes q_{a}^{i j}\left(\Gamma_{i j}\right)_{\beta}^{\alpha} \zeta_{\eta_{1}, \eta_{2}, \eta_{3}}^{\beta}
$$

Finally, to calculate the term $\theta \varepsilon$, the equations along various directions are,

$$
\text { (m) } \quad \gamma_{m} \sum_{\eta_{1}, \eta_{2}, \eta_{3}} \chi^{\eta_{1}, \eta_{2}, \eta_{3}} \otimes \frac{1}{2} g_{a}^{i}\left(\Gamma^{i}\right)_{\beta}^{\alpha}\left(\tau^{(01 a)} \zeta^{\beta}\right)_{\eta_{1}, \eta_{2}, \eta_{3}}
$$


$(p)$

$$
\begin{array}{ll}
(p) & \gamma_{p} \sum_{\eta_{1}, \eta_{2}, \eta_{3}} \chi^{\eta_{1}, \eta_{2}, \eta_{3}} \otimes\left(-\frac{i}{2}\right) h_{a}^{i}\left(\Gamma^{i}\right)_{\beta}^{\alpha}\left(\tau^{(10 a)} \zeta^{\beta}\right)_{\eta_{1}, \eta_{2}, \eta_{3}} \\
(a) \quad & \sum_{\eta_{1}, \eta_{2}, \eta_{3}} \chi^{\eta_{1}, \eta_{2}, \eta_{3}} \otimes\left(\frac{1}{2} g_{a}^{i}\left(\Gamma^{i}\right)_{\beta}^{\alpha}\left(\tau^{(100)} \zeta^{\beta}\right)_{\eta_{1}, \eta_{2}, \eta_{3}}-\frac{i}{2} h_{a}^{i}\left(\Gamma^{i}\right)_{\beta}^{\alpha}\left(\tau^{(010)} \zeta^{\beta}\right)_{\eta_{1}, \eta_{2}, \eta_{3}}\right)
\end{array}
$$

Combining all the terms, we recover the reduced gravitino BPS equations of (3.16),

$$
\begin{aligned}
(m) \quad 0 & =\frac{1}{f_{1}} \tau^{(300)} \zeta^{\alpha}+\frac{D_{a} f_{1}}{f_{1}} \tau^{(11 a)} \zeta^{\alpha}+g_{a}^{i} \tau^{(01 a)}\left(\Gamma^{i}\right)_{\beta}^{\alpha} \zeta^{\beta} \\
(p) \quad 0 & =\frac{i}{f_{2}} \tau^{(130)} \zeta^{\alpha}+\frac{D_{a} f_{2}}{f_{2}} \tau^{(11 a)} \zeta^{\alpha}-i h_{a}^{i} \tau^{(10 a)}\left(\Gamma^{i}\right)_{\beta}^{\alpha} \zeta^{\beta} \\
(a) \quad 0 & =\left(D_{a}+\frac{i}{2} \hat{\omega}_{a} \tau^{(003)}\right) \zeta^{\alpha}-\frac{1}{4} q_{a}^{i j}\left(\Gamma_{i j}\right)_{\beta}^{\alpha} \zeta^{\beta}+\frac{1}{2} g_{a}^{i} \tau^{(100)}\left(\Gamma^{i}\right)_{\beta}^{\alpha} \zeta^{\beta}-\frac{i}{2} h_{a}^{i} \tau^{(010)}\left(\Gamma^{i}\right)^{\alpha}{ }_{\beta} \zeta^{\beta}
\end{aligned}
$$

\section{B.2 Reduction of the $\chi^{r \alpha}$ BPS equation}

The $\chi^{r \alpha}$ equation is

$$
0=\frac{1}{\sqrt{2}} \gamma^{M} P_{M}^{i r}\left(\Gamma^{i}\right)^{\alpha}{ }_{\beta} \epsilon^{\beta}+\frac{1}{12} \gamma^{M N P} H_{M N P}^{r} \epsilon^{\alpha}
$$

The first term gives,

$$
\sum_{\eta_{1}, \eta_{2}, \eta_{3}} \chi^{\eta_{1}, \eta_{2}, \eta_{3}} \otimes \frac{1}{\sqrt{2}} p_{a}^{i r}\left(\Gamma^{i}\right)_{\beta}^{\alpha}\left(\tau^{(11 a)} \zeta^{\beta}\right)_{\eta_{1}, \eta_{2}, \eta_{3}}
$$

The second term gives,

$$
\sum_{\eta_{1}, \eta_{2}, \eta_{3}} \chi^{\eta_{1}, \eta_{2}, \eta_{3}} \otimes\left(-\frac{1}{2} \tilde{g}_{a}^{r}\left(\tau^{(01 a)} \zeta^{\alpha}\right)_{\eta_{1}, \eta_{2}, \eta_{3}}+\frac{i}{2} \tilde{h}_{a}^{r}\left(\tau^{(10 a)} \zeta^{\alpha}\right)_{\eta_{1}, \eta_{2}, \eta_{3}}\right)
$$

Combining all contributions, we recover the reduced spinor BPS equations of (3.17),

$$
0=\frac{1}{\sqrt{2}} p_{a}^{i r} \tau^{(11 a)}\left(\Gamma^{i}\right)^{\alpha}{ }_{\beta} \zeta^{\beta}-\frac{1}{2} \tilde{g}_{a}^{r} \tau^{(01 a)} \zeta^{\alpha}+\frac{i}{2} \tilde{h}_{a}^{r} \tau^{(10 a)} \zeta^{\alpha}
$$




\section{References}

[1] J. M. Maldacena, "The large N limit of superconformal field theories and supergravity," Adv. Theor. Math. Phys. 2 (1998) 231 [Int. J. Theor. Phys. 38 (1999) 1113] [arXiv:hep-th/9711200].

[2] S. S. Gubser, I. R. Klebanov and A. M. Polyakov, "Gauge theory correlators from non-critical string theory," Phys. Lett. B 428 (1998) 105 [arXiv:hep-th/9802109].

[3] E. Witten, "Anti-de Sitter space and holography," Adv. Theor. Math. Phys. 2 (1998) 253 [arXiv:hep-th/9802150].

[4] O. Aharony, S. S. Gubser, J. M. Maldacena, H. Ooguri and Y. Oz, "Large N field theories, string theory and gravity," Phys. Rept. 323, 183 (2000) [arXiv:hep-th/9905111].

[5] E. D'Hoker, D. Z. Freedman, "Supersymmetric gauge theories and the AdS / CFT correspondence," [hep-th/0201253].

[6] E. D'Hoker, J. Estes, M. Gutperle, D. Krym, P. Sorba, "Half-BPS supergravity solutions and superalgebras," JHEP 0812, 047 (2008). [arXiv:0810.1484 [hep-th]].

[7] H. Lin, O. Lunin and J. M. Maldacena, "Bubbling AdS space and 1/2 BPS geometries," JHEP 0410 (2004) 025 [arXiv:hep-th/0409174].

[8] E. D'Hoker, J. Estes and M. Gutperle, "Gravity duals of half-BPS Wilson loops," JHEP 0706 (2007) 063 [arXiv:0705.1004 [hep-th]].

[9] S. Yamaguchi, "Bubbling geometries for half BPS Wilson lines," Int. J. Mod. Phys. A 22 (2007) 1353 [arXiv:hep-th/0601089].

[10] J. Gomis and F. Passerini, "Holographic Wilson loops," JHEP 0608 (2006) 074 [arXiv:hepth/0604007].

[11] O. Lunin, "On gravitational description of Wilson lines," JHEP 0606, 026 (2006) [arXiv:hepth/0604133].

[12] E. D'Hoker, J. Estes and M. Gutperle, "Exact half-BPS Type IIB interface solutions I: Local solution and supersymmetric Janus," JHEP 0706 (2007) 021 [arXiv:0705.0022 [hep-th]].

[13] E. D'Hoker, J. Estes and M. Gutperle, "Exact half-BPS type IIB interface solutions. II: Flux solutions and multi-janus," JHEP 0706 (2007) 022 [arXiv:0705.0024 [hep-th]].

[14] C. Bachas, J. de Boer, R. Dijkgraaf and H. Ooguri, "Permeable conformal walls and holography," JHEP 0206, 027 (2002) [arXiv:hep-th/0111210].

[15] J. P. Gauntlett, D. Martelli, J. Sparks, D. Waldram, "Supersymmetric AdS(5) solutions of type IIB supergravity," Class. Quant. Grav. 23, 4693-4718 (2006). [hep-th/0510125].

[16] A. Clark and A. Karch, "Super Janus," JHEP 0510, 094 (2005) [arXiv:hep-th/0506265]. 
[17] E. D'Hoker, J. Estes and M. Gutperle, "Interface Yang-Mills, supersymmetry, and Janus," Nucl. Phys. B 753 (2006) 16 [arXiv:hep-th/0603013].

[18] J. Gomis and C. Romelsberger, "Bubbling defect CFT's," JHEP 0608 (2006) 050 [arXiv:hepth/0604155].

[19] D. Bak, M. Gutperle and S. Hirano, "A dilatonic deformation of AdS(5) and its field theory dual," JHEP 0305 (2003) 072 [arXiv:hep-th/0304129].

[20] O. Lunin, "1/2-BPS states in M theory and defects in the dual CFTs," JHEP 0710, 014 (2007) [arXiv:0704.3442 [hep-th]].

[21] E. D'Hoker, J. Estes, M. Gutperle and D. Krym, "Exact Half-BPS Flux Solutions in M-theory I, Local Solutions," JHEP 0808 (2008) 028 [arXiv:0806.0605 [hep-th]].

[22] E. D'Hoker, J. Estes, M. Gutperle and D. Krym, "Exact Half-BPS Flux Solutions in M-theory II: Global solutions asymptotic to $A d S_{7} \times S^{4}$," JHEP 0812 (2008) 044 [arXiv:0810.4647 [hepth]].

[23] E. D’Hoker, J. Estes, M. Gutperle and D. Krym, "Janus solutions in M-theory," JHEP 0906 (2009) 018 [arXiv:0904.3313 [hep-th]].

[24] E. D'Hoker, J. Estes, M. Gutperle and D. Krym, "Exact Half-BPS Flux Solutions in M-theory III: Existence and rigidity of global solutions asymptotic to $A d S 4 \times S 7$," arXiv:0906.0596 [hepth].

[25] Arthur L. Besse, Einstein Manifolds, Springer 1986.

[26] E. D'Hoker, J. Estes, "Integrable systems from supergravity BPS equations," JHEP 0809, 111 (2008). [arXiv:0807.3728 [hep-th]].

[27] D. Martelli, J. F. Morales, "Bubbling AdS(3)," JHEP 0502, 048 (2005). [hep-th/0412136].

[28] J. T. Liu, D. Vaman, "Bubbling 1/2 BPS solutions of minimal six-dimensional supergravity," Phys. Lett. B642, 411-419 (2006). [hep-th/0412242].

[29] J. P. Gauntlett, N. Kim, D. Waldram, "Supersymmetric $\operatorname{AdS}(3)$, AdS(2) and Bubble Solutions," JHEP 0704, 005 (2007). [hep-th/0612253].

[30] M. Boni and P. J. Silva, "Revisiting the D1/D5 system or bubbling in AdS(3)," JHEP 0510 (2005) 070 [arXiv:hep-th/0506085].

[31] M. Chiodaroli, M. Gutperle and D. Krym, "Half-BPS Solutions locally asymptotic to $A d S_{3} \times S^{3}$ and interface conformal field theories," JHEP 1002 (2010) 066 [arXiv:0910.0466 [hep-th]].

[32] M. Chiodaroli, E. D'Hoker, M. Gutperle, "Open Worldsheets for Holographic Interfaces," JHEP 1003, 060 (2010). [arXiv:0912.4679 [hep-th]]. 
[33] J. Kumar and A. Rajaraman, "New supergravity solutions for branes in $A d S_{3} \times S^{3}$," Phys. Rev. D 67 (2003) 125005 [arXiv:hep-th/0212145].

[34] J. Kumar and A. Rajaraman, "Supergravity solutions for $A d S_{3} \times S^{3}$ branes," Phys. Rev. D 69 (2004) 105023 [arXiv:hep-th/0310056].

[35] J. Kumar and A. Rajaraman, "Revisiting D-branes in $A d S_{3} \times S^{3}$," Phys. Rev. D 70 (2004) 105002 [arXiv:hep-th/0405024].

[36] O. Lunin, "Brane webs and 1/4-BPS geometries," JHEP 0809 (2008) 028 [arXiv:0802.0735 [hep-th]].

[37] M. Chiodaroli, M. Gutperle, L. -Y. Hung, D. Krym, "String Junctions and Holographic Interfaces," Phys. Rev. D83, 026003 (2011). [arXiv:1010.2758 [hep-th]].

[38] K. Dasgupta, S. Mukhi, "BPS nature of three string junctions," Phys. Lett. B423, 261-264 (1998). [hep-th/9711094].

[39] A. Sen, "String network," JHEP 9803, 005 (1998). [hep-th/9711130].

[40] L. J. Romans, "Selfduality For Interacting Fields: Covariant Field Equations For SixDimensional Chiral Supergravities," Nucl. Phys. B 276 (1986) 71.

[41] S. Deger, A. Kaya, E. Sezgin and P. Sundell, "Spectrum of D =6, N = 4b supergravity on AdS(3) x S(3)," Nucl. Phys. B 536 (1998) 110 [arXiv:hep-th/9804166].

[42] S. D. Avramis, "Anomaly-free supergravities in six dimensions," arXiv:hep-th/0611133.

[43] Y. Tanii, "N=8 Supergravity In Six-Dimensions," Phys. Lett. B 145 (1984) 197.

[44] C. M. Hull, "Strongly coupled gravity and duality," Nucl. Phys. B583, 237-259 (2000). [hepth/0004195].

[45] F. Giani, M. Pernici and P. van Nieuwenhuizen, "Gauged N=4 D =6 Supergravity," Phys. Rev. D 30 (1984) 1680.

[46] D. Marolf, "Chern-Simons terms and the three notions of charge," [hep-th/0006117].

[47] Y. Guo, University of California Los Angeles Ph.D Thesis, to appear.

[48] D. Gaiotto, E. Witten, "S-Duality of Boundary Conditions In N=4 Super Yang-Mills Theory," [arXiv:0807.3720 [hep-th]].

[49] D. Gaiotto, E. Witten, "Supersymmetric Boundary Conditions in N=4 Super Yang-Mills Theory," [arXiv:0804.2902 [hep-th]].

[50] D. Gaiotto, E. Witten, "Janus Configurations, Chern-Simons Couplings, And The theta-Angle in N=4 Super Yang-Mills Theory," JHEP 1006, 097 (2010). [arXiv:0804.2907 [hep-th]]. 
[51] A. B. Clark, D. Z. Freedman, A. Karch, M. Schnabl, "The Dual of Janus -:- an interface CFT," Phys. Rev. D71, 066003 (2005). [hep-th/0407073].

[52] O. Aharony, L. Berdichevsky, M. Berkooz and I. Shamir, "Near-horizon solutions for D3-branes ending on 5-branes," arXiv:1106.1870 [hep-th].

[53] B. Assel, C. Bachas, J. Estes and J. Gomis, "Holographic Duals of D=3 N=4 Superconformal Field Theories," arXiv:1106.4253 [hep-th].

[54] A. Karch, L. Randall, "Open and closed string interpretation of SUSY CFT's on branes with boundaries," JHEP 0106 (2001) 063. [hep-th/0105132].

[55] T. Takayanagi, "Holographic Dual of BCFT," [arXiv:1105.5165 [hep-th]].

[56] M. Chiodaroli, M. Gutperle and L. Y. Hung, "Boundary entropy of supersymmetric Janus solutions," JHEP 1009, 082 (2010) [arXiv:1005.4433 [hep-th]].

[57] S. Ryu and T. Takayanagi, "Holographic derivation of entanglement entropy from AdS/CFT," Phys. Rev. Lett. 96 (2006) 181602 [arXiv:hep-th/0603001]. 